\title{
The inverse spectral problem for self-adjoint Hankel operators
}

\author{
by
}

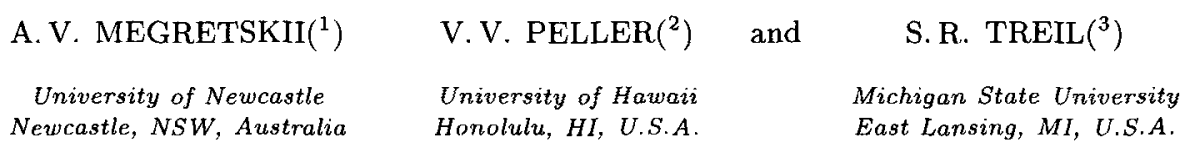

\section{Contents}

Introduction . . . . . . . . . . . . . . . . . . . . . . . . 242

I. Necessary conditions . . . . . . . . . . . . . . . . . . 248

I.1. Spectral multiplicities of self-adjoint Hankel operators . . . 249

I.2. Eigenvalues of Hankel operators . . . . . . . . . . 253

II. Balanced realizations with continuous time . . . . . . . . . . 255

II.1. Hankel operator and balanced realizations . . . . . . . . 256

II.2. Unitary equivalence modulo the kernel . . . . . . . . . . 257

II.3. Lyapunov equations . . . . . . . . . . . . . . . . . . . . 260

II.4. The main construction . . . . . . . . . . . . . 262

II.5. The kernel of $\Gamma_{h} \ldots \ldots \ldots \ldots \ldots$

II.6. Exercises in measure theory . . . . . . . . . . . . . . 275

11.7. Positive Hankel operators with multiple spectrum . . . . 277

III. Balanced realizations with discrete time . . . . . . . . . . 279

III. 1. Hankel operators and linear systems . . . . . . . . . . . . 280

III.2. Output normal realizations of Hankel operators . . . . . . 281

III.3. Balanced realizations ... . . . . . . . . . . . . . . . 284

III.4. Asymptotic stability . . . . . . . . . . . . . . 288

III.5. The main construction . . . . . . . . . . . . . . . . 289

III.6. The point spectrum of $A^{*}$ on the unit circle . . . . . . 294

III.7. How to cope with $Q \ldots \ldots \ldots \ldots \ldots \ldots$

III.8. The Fredholm spectrum of $\mathcal{J} J \ldots \ldots \ldots$

III.9. Compact integral operators . . . . . . . . . . 300

III.10. A theorem in perturbation theory . . . . . . . . . . . 306

Refererences ....................... 308

(1) Also affiliated to St. Petersburg University, St. Petersburg, Russia.

(') Also affiliated to the St. Petersburg Branch of the Steklov Mathematical Institute, St. Petersburg, Russia. Research supported by an NSF grant in modern analysis.

( $\left.{ }^{3}\right)$ Also affiliated to St. Petersburg University, St. Petersburg, Russia. Research supported by an NSF grant in modern analysis. 


\section{Introduction}

In this paper we study spectral properties of self-adjoint Hankel operators. For the last years the theory of Hankel operators has been developing very intensively and many new applications have been found. Hankel operators are widely used in function theory, operator theory, approximation theory, prediction theory, and control theory (see [N], $[\mathrm{Po}],[\mathrm{Pa}],[\mathrm{PK}],[\mathrm{F}])$.

There are many different equivalent ways to define a Hankel operator. By a Hankel operator we mean a (bounded) operator $\Gamma$ on the sequence space $l^{2}$ which has a Hankel matrix in the standard basis $\left\{e_{j}\right\}_{j \geqslant 0}$, i.e. $\left(\Gamma e_{j}, e_{k}\right)=\alpha_{j+k}, j, k \geqslant 0$, where $\left\{\alpha_{j}\right\}_{j \geqslant 0}$ is a sequence of complex numbers. Let $S$ be the shift operator on $l^{2}$, i.e. $S e_{j}=e_{j+1}, j \geqslant 0$. It is easy to see that a bounded operator $\Gamma$ on $l^{2}$ is a Hankel operator if and only if it satisfies the commutation relation

$$
S^{*} \Gamma=\Gamma S .
$$

The main aim of this paper is to describe the class of self-adjoint Hankel operators in spectral terms. To be more precise we obtain in this paper a characterization of those self-adjoint operators that are unitarily equivalent to a Hankel operator. Clearly an operator $\Gamma$ is unitarily equivalent to a Hankel operator if and only if there exists an orthonormal basis $\left\{e_{j}\right\}_{j \geqslant 0}$ such that $\left(\Gamma e_{j}, e_{k}\right)=\alpha_{j+k}, j, k \geqslant 0$, for a sequence $\left\{\alpha_{j}\right\}_{j \geqslant 0}$ of complex numbers. It is also obvious that $\Gamma$ is self-adjoint if and only if $\alpha_{j} \in \mathbf{R}$ for any $j \geqslant 0$.

We arrived at this problem in the following way. In [KP] the following question was studied. Let $\mathcal{L}, \mathcal{K}$ be subspaces of a Hilbert space $\mathcal{H}$. The problem was to find out under which conditions on $\mathcal{L}$ and $\mathcal{K}$ there exists a stationary process $\left\{x_{n}\right\}_{n \in \mathbf{Z}}$ in $\mathcal{H}$ whose past

$$
\operatorname{span}\left\{x_{j}: j<0\right\}
$$

coincides with $\mathcal{L}$ and whose future

$$
\operatorname{span}\left\{x_{j}: j \geqslant 0\right\}
$$

coincides with $\mathcal{K}$ (by "span" we mean "closed linear span"). Here a stationary process is a two-sided sequence $\left\{x_{n}\right\}_{n \in Z}$ of elements of $\mathcal{H}$ such that the inner products $\left(x_{j}, x_{k}\right)$ depend only on $j-k$.

It was shown in [KP] that the above problem is equivalent to the following one. Let $K$ be a non-negative self-adjoint operator on Hilbert space. Under which conditions does there exist a Hankel operator $\Gamma$ whose modulus $\left(\Gamma^{*} \Gamma\right)^{1 / 2}$ is unitarily equivalent to $K$ ? The following simple necessary conditions were found in [KP]:

(i) $\operatorname{Ker} K$ is either trivial or infinite-dimensional;

(ii) $K$ is non-invertible. 
The condition (i) follows immediately from Beurling's theorem (see [N, Chapter 1]). Indeed it is easily seen from (1) that if $\Gamma$ is a Hankel operator, then Ker $\Gamma$ is an invariant subspace of $S$ and so by Beurling's theorem $\operatorname{Ker} K$ is either trivial or infinite-dimensional.

The condition (ii) follows immediately from the fact that if $\Gamma$ is a Hankel operator and $\left(\Gamma e_{j}, e_{k}\right)=\alpha_{j+k}, j, k \geqslant 0$, then

$$
\left\|\Gamma e_{k}\right\|^{2}=\sum_{j \geqslant k}\left|\alpha_{j}\right|^{2} \rightarrow 0 \quad \text { as } k \rightarrow \infty .
$$

A question was posed in [KP] of whether the conditions (i) and (ii) are also sufficient.

The first step was made in [T1]. It was shown there that if $K$ satisfies (i) and (ii) and in addition has simple discrete spectrum, then $K$ is unitarily equivalent to the modulus of a Hankel operator. Then, it was shown in [TV] that each non-negative operator with discrete spectrum that satisfies (i) and (ii) is unitarily equivalent to the modulus of a Hankel operator.

The next step was made by $\mathrm{R}$. Ober in $[\mathrm{O} 1]$ and [O2]. He found a new approach to the problem which is based on the so-called balanced realizations with continuous time. Using that approach R. Ober obtained a different proof of the results of [TV] "modulo the kernel". However, it turned out that Ober's approach works in a much more general situation and with its help it was proved in [T2] that the conditions (i) and (ii) are also sufficient.

Let us dwell briefly on the above approach. Let $\mathcal{K}$ be a Hilbert space (state space), $A$ an operator on $\mathcal{K}, B$ an operator from $\mathbf{C}$ to $\mathcal{K}$ and $C$ an operator from $\mathcal{K}$ to $\mathbf{C}$. Consider the following linear dynamical system with one-dimensional input and one-dimensional output:

$$
\left\{\begin{array}{l}
x^{\prime}(t)=A x(t)+B u(t) \\
y(t)=C x(t)
\end{array}\right.
$$

Let $b, c$ be the vectors in $\mathcal{K}$ such that $B u=u b, b \in \mathbf{C}, C x=(x, c), x \in \mathcal{K}$. With the above linear system one can associate the (formal) Hankel operator $\Gamma_{h}$ :

$$
\left(\Gamma_{h} f\right)(t)=\int_{0}^{\infty} h(s+t) f(s) d s, \quad f \in L^{2}(\mathbf{R})
$$

where $h(t)=\left(e^{t A} b, c\right)$.

The operator $\Gamma_{h}$ need not be bounded in general but it is bounded under certain natural conditions. Note that $\Gamma_{h}$ is not only an analogue of a Hankel matrix but also unitarily equivalent to a Hankel operator. It is well known that the matrix of $\Gamma_{h}$ in the basis of Laguerre functions is Hankel.

The idea behind the approach in question is to choose a triple $\{A, B, C\}$ so that the modulus of the corresponding operator $\Gamma_{h}$ be unitarily equivalent to the given operator $K$. 
The solution obtained in [T2] allows one to construct a self-adjoint Hankel operator whose modulus is unitarily equivalent to $\Gamma$. Therefore the problem we study in this paper is a refinement of the above problem posed in [KP]. Namely, the results of [T2] show that the spectral multiplicity function $\nu_{|\Gamma|}$ (a precise definition is given below) of the modulus of a self-adjoint Hankel operator $\Gamma$ can be an arbitrary function $\nu$ which satisfies the conditions:

(i) $\nu(0)=0$ or $\nu(0)=\infty$;

(ii) $0 \in \operatorname{supp} \nu$.

Clearly $\nu_{|\Gamma|}(t)=\nu_{\Gamma}(t)+\nu_{\Gamma}(-t), t>0$. So the problem we study in this paper is to investigate in which way the value of $\nu_{|\Gamma|}(t)$ can be distributed between $\nu_{\Gamma}(t)$ and $\nu_{\Gamma}(-t)$.

To formulate our main result we need the notion of von Neumann (direct) integral (see [BS1, Chapter 7] for more detail). Let $\mu$ be a finite positive Borel measure on $\mathbf{R}$ and $\{\mathcal{H}(t)\}_{t \in \mathbf{R}}$ a measurable family of Hilbert spaces. That means that we are given an at most countable set $\Omega$ of functions $f$ such that $f(t) \in \mathcal{H}(t), \mu$-a.e.,

$$
\operatorname{span}\{f(t): f \in \Omega\}=\mathcal{H}(t) \quad \text { for } \mu \text {-almost all } t,
$$

and the function

$$
t \mapsto\left(f_{1}(t), f_{2}(t)\right)_{\mathcal{H}(t)}
$$

is $\mu$-measurable for any $f_{1}, f_{2} \in \Omega$. A function $g$ with values $g(t)$ in $\mathcal{H}(t)$ is called measurable if the scalar-valued function $t \mapsto(f(t), g(t))_{\mathcal{H}(t)}$ is measurable for any $f \in \Omega$.

The von Neumann integral (direct integral) $\int \bigoplus \mathcal{H}(t) d \mu(t)$ consists of measurable functions $f, f(t) \in \mathcal{H}(t)$, such that

$$
\|f\|=\left(\int\|f(t)\|_{\mathcal{H}(t)}^{2} d \mu(t)\right)^{1 / 2}<\infty
$$

If $f, g \in \int \oplus \mathcal{H}(t) d \mu(t)$, then their inner product is defined by

$$
(f, g)=\int(f(t), g(t)) d \mu(t)
$$

By von Neumann's theorem (see [BS1]) each self-adjoint operator on a separable Hilbert space is unitarily equivalent to multiplication by the independent variable on a direct integral $\int \bigoplus \mathcal{H}(t) d \mu(t)$ :

$$
(A f)(t)=t f(t), \quad f \in \int \bigoplus \mathcal{H}(t) d \mu(t)
$$

Without los- if generality we can assume that $\mathcal{H}(t) \neq 0, \mu$-almost everywhere. In this case $\mu$ is called a scalar spectral measure of $A$. The spectral multiplicity function $\nu_{A}$ of the operator $A$ is defined $\mu$-almost everywhere by

$$
\nu_{A}(t)=\operatorname{dim} \mathcal{H}(t)
$$


It is well known (see [BS1]) that self-adjoint operators $A_{1}$ and $A_{2}$ are unitarily equivalent if and only if their scalar spectral measures are mutually absolutely continuous and $\nu_{A_{1}}=\nu_{A_{2}}$ almost everywhere.

If $A$ is a self-adjoint operator with scalar spectral measure $\mu$ and spectral multiplicity function $\nu_{A}$, then $A$ is unitarily equivalent to multiplication by the independent variable on

$$
\int \bigoplus \mathcal{H}(t) d \mu(t)
$$

where $\mu$ is a scalar spectral measure of $A$, and the $\mathcal{H}(t)$ are embedded in a Hilbert space $E$ with basis $\left\{e_{j}\right\}_{j \geqslant 1}$ and

$$
\mathcal{H}(t)=\operatorname{span}\left\{e_{j}: 1 \leqslant j<\nu_{A}(t)+1\right\}
$$

Now we are in a position to formulate our main result. As usual, $\mu_{a}$ and $\mu_{s}$ are the absolutely continuous and the singular component, respectively, of $\mu$.

THEOREM 1. Let $\Gamma$ be a bounded self-adjoint operator on Hilbert space, $\mu$ a scalar spectral measure of $\Gamma$, and $\nu$ its spectral multiplicity function. Then $\Gamma$ is unitarily equivalent to a Hankel operator if and only if the following conditions hold:

(C1) either $\operatorname{Ker} \Gamma=\{0\}$ or $\operatorname{dim} \operatorname{Ker} \Gamma=\infty$;

(C2) $\Gamma$ is non-invertible;

(C3) $|\nu(t)-\nu(-t)| \leqslant 2, \mu_{a}-a . e$, and $|\nu(t)-\nu(-t)| \leqslant 1, \mu_{s}$-a.e.

Note here that (C3) means in particular that if one of the numbers $\nu(t)$ and $\nu(-t)$ is infinite, then the other one must also be infinite.

We have to say a few words here to justify the meaning of the inequalities in (C3). Let $\tilde{\mu}$ be the measure defined by $\tilde{\mu}(\Delta)=\mu(\Delta)+\mu(-\Delta)$. We can assume that the function $\nu$ is defined $\tilde{\mu}$-almost everywhere. If $\delta$ is a Borel set such that $\mu(\delta)=0$, then $\nu(t)=0$ on $\delta$, $\tilde{\mu}$-almost everywhere. With this convention $\nu(t)-\nu(-t)$ is defined $\tilde{\mu}$-almost everywhere which makes it possible to justify the left-hand sides of the inequalities in (C3).

The necessity of the conditions (C1) and (C2) is equivalent to the necessity of the above conditions (i) and (ii) for the modulus of a Hankel operator. In Chapter I of this paper we prove that $(\mathrm{C} 3)$ is also necessary. It is easy to see that (C3) implies the following inequality for the multiplicities of eigenvalues of a self-adjoint Hankel operator $\Gamma$ : if $\lambda \in \mathbf{R}$, then

$$
|\operatorname{dim} \operatorname{Ker}(\Gamma-\lambda I)-\operatorname{dim} \operatorname{Ker}(\Gamma+\lambda I)| \leqslant 1
$$

(if one of the dimensions is infinite, then the other one must also be infinite).

For compact self-adjoint operators (3) was proved in [Pe] by another method. Earlier in $[\mathrm{C}]$ a weaker inequality was obtained. In $[\mathrm{HW}]$ another method was used to prove (3) 
for compact self-adjoint Hankel operators. V. M. Adamyan told us that he also obtained another proof of (3) in the compact case but had not published the proof.

In $\S I .2$ we obtain a very simple proof of (3) for an arbitrary Hankel operator $\Gamma$ (not necessarily self-adjoint) and an arbitrary $\lambda \in \mathbf{C}$.

In Chapter II we use linear systems (2) to construct a Hankel operator with prescribed spectral properties. We prove that if $\Gamma$ is a self-adjoint operator which satisfies (C1), (C2), and the condition

$$
|\nu(t)-\nu(-t)| \leqslant 1, \quad \mu \text {-a.e. }
$$

then there exists a system (2) (in fact a balanced system, see §II.1 for a definition) such that the Hankel operator associated with it is unitarily equivalent to $\Gamma$. Explicit formulae are obtained for the operators $A, B, C$ in (2).

However, we also show in Chapter II that if $\Gamma$ is a positive self-adjoint operator with multiple spectrum, then there exists no balanced system (2) for which the corresponding Hankel operator is unitarily equivalent to $\Gamma$. This shows that in contrast with the problem of the description of possible moduli of Hankel operators, the balanced systems (2) cannot produce enough Hankel operators to solve our problem completely.

Note here that we consider only those linear systems (2) which involve bounded operators $A, B, C$. It is also possible to consider linear systems for which $A$ is the generator of a contractive semigroup, $B$ is an operator from $\mathbf{C}$ to a Hilbert space which is larger than $\mathcal{K}$, and $C$ is a linear functional defined on a dense subset of $\mathcal{K}$. Using the results of $[\mathrm{Y}], \mathrm{R}$. Ober and S. Montgomery-Smith $[\mathrm{OM}]$ proved that for any bounded Hankel operator $\Gamma$ on $L^{2}\left(\mathbf{R}_{+}\right)$there exists a generalized balanced linear system such that the Hankel operator associated with it coincides with $\Gamma$ (see also $[\mathrm{S}]$ ). However we do not know how to evaluate the spectra of Hankel operators associated with systems that have unbounded $B$ and $C$.

Note that positive Hankel operators with multiple spectra do exist. A classical example of such an operator is the Carleman operator $K$ defined on $L^{2}\left(\mathbf{R}_{+}\right)$by

$$
(K f)(s)=\int_{0}^{\infty} \frac{f(t)}{s+t} d t
$$

(see $[\mathrm{Po}, \S 2.6])$. Other interesting examples of such operators are given by J. Howland in [Ho1], [Ho2], and [Ho3].

In Chapter III, we prove that the conditions (C1)-(C3) are sufficient. The method of the proof is based on linear dynamical systems with discrete time. Let $A$ be an operator on a Hilbert space $\mathcal{K}$ and let $B u=u b, u \in \mathbf{C}, C x=(x, c), x \in \mathcal{H}$, where $b, c \in \mathcal{H}$. Consider the linear system:

$$
\left\{\begin{array}{l}
x_{n+1}=A x_{n}+B u_{n}, \\
y_{n}=C x_{n} .
\end{array}\right.
$$


As in the case of continuous time we can associate with this system the Hankel matrix $\left\{\alpha_{j+k}\right\}_{j, k \geqslant 0}$, where $\alpha_{j}=\left(A^{j} b, c\right), j \geqslant 0$. N. Young proved in [Y] that for any bounded Hankel matrix $\left\{\alpha_{j+k}\right\}_{j, k \geqslant 0}$ there exists a balanced linear system (4) (see $\S I I I .1$ for a definition) such that $\alpha_{j}=\left(A^{j} b, c\right)$.

The main result of Chapter III claims that if $\Gamma$ is a self-adjoint operator satisfying the conditions (C1)-(C3), then there exists a balanced linear system (4) such that the corresponding Hankel operator is unitarily equivalent to $\Gamma$.

We can consider also the problem of spectral characterization of self-adjoint blockHankel operators of order $n$, i.e. block-Hankel matrices of the form $\left\{\alpha_{j+k}\right\}_{j, k \geqslant 0}$, where $\alpha_{j}$ is an $n \times n$ matrix for every $j$. The following analogue of Theorem 1 holds.

Theorem 2. Let $\Gamma$ be a bounded self-adjoint operator on Hilbert space, $\mu$ a scalar spectral measure of $\Gamma$, and $\nu$ its spectral multiplicity function. Then $\Gamma$ is unitarily equivalent to a block-Hankel operator of order $n$ if and only if the following conditions are satisfied:

(C1) either $\operatorname{Ker} \Gamma=\{\mathbf{0}\}$ or $\operatorname{dim} \operatorname{Ker} \Gamma=\infty$;

(C2) $\Gamma$ is non-invertible;

$\left(\mathrm{C} 3_{n}\right)|\nu(t)-\nu(-t)| \leqslant 2 n, \mu_{a}-a . e .$, and $|\nu(t)-\nu(-t)| \leqslant n, \mu_{s}-a . e$.

The sufficiency follows easily from Theorem 1 and the fact that if $\Gamma$ satisfies (C1)$\left(\mathrm{C} 3_{n}\right)$, then it can be represented as an orthogonal sum of $n$ operators each of which satisfies (C1)-(C3). The necessity can be proved by the same method as in the case $n=1$ (see Chapter I).

In what follows we need a description of the class of bounded operators which intertwine two given self-adjoint operators. Let $A_{1}, A_{2}$ be self-adjoint operators which are given as multiplications by the independent variable on direct integrals

$$
\int \bigoplus \mathcal{H}_{1}(t) d \mu_{1}(t) \text { and } \int \bigoplus \mathcal{H}_{2}(t) d \mu_{2}(t)
$$

The following result is apparently known to experts, however we were unable to find a reference.

LEMMA 3. Let $B$ be a bounded operator such that $B A_{1}=A_{2} B$. Then there is $a$ $\left(\mu_{1}+\mu_{2}\right)$-measurable operator-valued function $b$,

$$
b(t): \mathcal{H}_{1}(t) \rightarrow \mathcal{H}_{2}(t)
$$

such that

$$
(B f)(t)=b(t) f(t), \quad f \in \int \bigoplus \mathcal{H}_{1}(t) d \mu_{1}(t) .
$$


We have to say a few words to justify (5). We can assume that $\mathcal{H}_{1}(t)$ and $\mathcal{H}_{2}(t)$ are defined $\left(\mu_{1}+\mu_{2}\right)$-almost everywhere and if $\mu_{1}(\delta)=0\left(\mu_{2}(\delta)=0\right)$ for a Borel set $\delta$, then $\mathcal{H}_{1}(t)=\{\mathbf{0}\}\left(\mathcal{H}_{2}(t)=\{\mathbf{0}\}\right)$ on $\delta,\left(\mu_{1}+\mu_{2}\right)$-almost everywhere.

Proof. Let $\mu=\mu_{1}+\mu_{2}$. Without loss of generality we can assume that $\mu_{j}$ is the restriction of $\mu$ to the set

$$
\left\{t: \mathcal{H}_{j}(t) \neq\{\mathbf{0}\}\right\}, \quad j=1,2 .
$$

Let

Put

$$
\mathcal{H}_{j}=\int \bigoplus \mathcal{H}_{j}(t) d \mu_{1}(t)=\int \bigoplus \mathcal{H}_{j}(t) d \mu(t), \quad j=1,2
$$

$$
\mathcal{H}(t)=\mathcal{H}_{1}(t) \oplus \mathcal{H}_{2}(t) \quad \text { and } \quad \mathcal{H}=\int \oplus \mathcal{H}(t) d \mu(t)
$$

Then $\mathcal{H}_{1}$ and $\mathcal{H}_{2}$ are naturally embedded in $\mathcal{H}$. Define the self-adjoint operator $A$ on $\mathcal{H}$ as multiplication by the independent variable. Consider the operator $\widetilde{B}$ on $\mathcal{H}$ defined by

$$
\widetilde{B} f=B \mathcal{P}_{1} f
$$

where $\mathcal{P}_{1}$ is the orthogonal projection from $\mathcal{H}$ onto $\mathcal{H}_{1}$. It is easy to see that Range $\widetilde{B} \subset$ $\mathcal{H}_{2}, B=\widetilde{B} \mid \mathcal{H}_{1}$ and $B A=A B$. Then (see [BS1, Chapter 7]) there exists a measurable operator-valued function $\tilde{b}, \tilde{b}(t): \mathcal{H}(t) \rightarrow \mathcal{H}(t)$, such that $(\tilde{B} f)(t)=\tilde{b}(t) f(t)$. It is easy to see that $\tilde{b}(t) x=0$ for $x \in \mathcal{H}_{2}(t)$ and Range $\tilde{b}(t) \subset \mathcal{H}_{2}(t)$. We can now define $b(t)$ by $\tilde{b}(t) \mid \mathcal{H}_{1}(t)$. Clearly $b(t)$ is an operator from $\mathcal{H}_{1}(t)$ to $\mathcal{H}_{2}(t)$ and $(B f)(t)=b(t) f(t)$.

Concluding the introduction we would like to mention that in each chapter we use separate numeration of theorems, lemmas and displayed formulae.

We would like to express our deep gratitude to the referee for thoroughly reading the manuscript, finding a lot of misprints and making helpful suggestions.

\section{Necessary conditions}

The main result of this chapter is given in $\S I .1$. It will be shown there that if $\Gamma$ is a self-adjoint operator on Hilbert space which is unitarily equivalent to a Hankel operator, then $\Gamma$ satisfies the conditions $(\mathrm{C} 1),(\mathrm{C} 2)$, and $(\mathrm{C} 3)$ in the Introduction.

In $\S I .2$ we consider the problem of comparison of the multiplicities of the eigenvalues $\lambda$ and $-\lambda, \lambda \in \mathbf{C}$, for an arbitrary (not necessarily self-adjoint) Hankel operator $\Gamma$. Certainly it follows from the results of $\S I .1$ that if $\Gamma$ is a self-adjoint Hankel operator and $\lambda \in \mathbf{R}$, then

$$
|\operatorname{dim} \operatorname{Ker}(\Gamma-\lambda I)-\operatorname{dim} \operatorname{Ker}(\Gamma+\lambda I)| \leqslant 1 .
$$

It will be shown in $§ I .2$ that the same is true for an arbitrary Hankel operator $\Gamma$ and any $\lambda \in \mathbf{C}$. 


\section{I.1. Spectral multiplicities of self-adjoint Hankel operators}

Let $\Gamma$ be a self-adjoint operator on a Hilbert space $\mathcal{H}$. Then (see the Introduction) $\Gamma$ admits the following realization. There exist a finite Borel measure $\mu$ on the real line and a measurable family of Hilbert spaces $\mathcal{H}(t), t \in \mathbf{R}$, such that $\mathcal{H}(t) \neq\{\mathbf{0}\}, \mu$-almost everywhere, $\mathcal{H}$ can be identified with the direct integral

$$
\mathcal{H}=\int_{\mathbf{R}} \oplus \mathcal{H}(t) d \mu(t)
$$

and under this identification

$$
(\Gamma f)(t)=t f(t), \quad f \in \mathcal{H}
$$

The spectral multiplicity function $\nu$ is defined by

$$
\nu(t)=\operatorname{dim} \mathcal{H}(t) .
$$

As we have mentioned in the Introduction, we can assume that $\nu$ is defined $\tilde{\mu}$-almost everywhere, where $\tilde{\mu}(\Delta) \stackrel{\text { def }}{=} \mu(\Delta)+\mu(-\Delta)$ for any Borel set $\Delta$. Then if $\delta$ is a Borel set such that $\mu(\delta)=0$, then $\nu(t)=0$ on $\delta, \tilde{\mu}$-almost everywhere.

Let $\mu=\mu_{a}+\mu_{s}$ be the Lebesgue decomposition of $\mu$, where $\mu_{a}$ is absolutely continuous and $\mu_{s}$ is singular with respect to Lebesgue measure.

The following theorem is the main result of this section.

THEOREM 1.1. Let $\Gamma$ be the self-adjoint operator (1.2) on the space (1.1). If $\Gamma$ is unitarily equivalent to a Hankel operator, then the following conditions are satisfied:

(C1) either $\operatorname{Ker} \Gamma=\{0\}$ or $\operatorname{dim} \operatorname{Ker} \Gamma=\infty$;

(C2) $\Gamma$ is non-invertible;

(C3) $|\nu(t)-\nu(-t)| \leqslant 2, \mu_{a}$-a.e., and $|\nu(t)-\nu(-t)| \leqslant 1, \mu_{s}$-a.e.

Remark. If $\nu(t)=\infty$ or $\nu(-t)=\infty$, then (C3) has to be understood as $\nu(t)=$ $\nu(-t)=\infty$.

As we have already mentioned in the Introduction, the conditions $(\mathrm{C} 1)$ and $(\mathrm{C} 2)$ are trivial. Thus we only have to prove (C3).

Before we proceed to the proof, we adopt the following terminology. If $f$ is a Borel function, $\varrho$ is a Borel measure, and $\Delta$ is a Borel set, we say $f$ is supported on $\Delta$ ( $\varrho$ is supported on $\Delta)$ if $f$ is zero almost everywhere outside $\Delta$ ( $\varrho$ is zero outside $\Delta$ ).

Proof of Theorem 1.1. Suppose that $\Gamma$ is unitarily equivalent to a Hankel operator. Then $\mathcal{H}$ has an orthonormal basis $\left\{e_{j}\right\}_{j \geqslant 0}$ such that $\Gamma$ has Hankel matrix in this basis, 
i.e. $\left(\Gamma e_{j}, e_{k}\right)=\alpha_{j+k}, j, k \geqslant 0$, where $\left\{\alpha_{j}\right\}_{j \geqslant 0}$ is a sequence of real numbers $\left(\alpha_{j} \in \mathbf{R}\right.$ since $\Gamma$ is self-adjoint).

Consider the shift operator $S$ on $\mathcal{H}$ defined by

$$
S \sum_{n \geqslant 0} x_{n} e_{n}=\sum_{n \geqslant 0} x_{n} e_{n+1} .
$$

Put $\alpha=\sum_{j=0}^{\infty} \alpha_{j} e_{j}=\Gamma e_{0}$. It is easy to see that the following commutation relations hold:

$$
\begin{aligned}
S^{*} \Gamma & =\Gamma S \\
S \Gamma f-\Gamma S^{*} f & =\left(f, e_{0}\right) S \alpha-(f, S \alpha) e_{0}, \quad f \in \mathcal{H} .
\end{aligned}
$$

Let us first prove the inequality $|\nu(t)-\nu(-t)| \leqslant 2, \mu$-a.e. Let $f$ be a function in $\mathcal{H}$ such that $f(t) \perp e_{0}(t)$ and $f(t) \perp(S \alpha)(t), \mu$-a.e. Consider the function $\left(S^{*}-S\right) f$. We have

$$
\Gamma\left(S^{*}-S\right) f=\Gamma S^{*} f-\Gamma S f=S \Gamma f-S^{*} \Gamma f=-\left(S^{*}-S\right) \Gamma f
$$

by (1.3) and (1.4), since $f \perp e_{0}$ and $f \perp S \alpha$.

Let us now make use of Lemma 3 from the Introduction. Put $B=S^{*}-S$. Let $A_{1}$ be the restriction of $\Gamma$ to

$$
\tilde{\mathcal{H}}=\left\{f \in \mathcal{H}: f(t) \perp e_{0}(t) \text { and } f(t) \perp(S \alpha)(t), \mu \text {-a.e. }\right\},
$$

and let $A_{2}=-\Gamma$. We have $B A_{1}=A_{2} B$ by (1.5). Therefore by Lemma 3 there exists a bounded weakly measurable function $b, b(t): \widetilde{\mathcal{H}}(t) \rightarrow \mathcal{H}(-t)$, such that

$$
(B f)(t)=b(t) f(-t)
$$

where

$$
\tilde{\mathcal{H}}(t)=\left\{x \in H(t): x \perp e_{0}(t) \text { and } x \perp(S \alpha)(t)\right\} .
$$

Let us show that $\operatorname{Ker} b(t)=\{0\}, \mu$-a.e. Indeed, assume that there exists a Borel set $\Delta, \mu(\Delta)>0$, such that $\operatorname{Ker} b(t) \neq\{0\}$ for any $t$ in $\Delta$. Let $P_{t}$ be the orthogonal projection of $\widetilde{H}(t)$ onto $\operatorname{Ker} b(t)$. Then the function $t \mapsto P_{t}$ is weakly measurable. This follows from the fact that

$$
P_{t}=\lim _{\varepsilon \rightarrow 0} \chi_{\varepsilon}\left(b^{*}(t) b(t)\right)
$$

where $\chi_{\varepsilon}(s)=0$ for $s>\varepsilon$ and $\chi_{\varepsilon}(s)=1$ for $0 \leqslant s \leqslant \varepsilon$. Therefore (see the Introduction) the spaces $\operatorname{Ker} b(t)$ form a measurable family in a natural way and we can consider the direct integral

$$
\int \bigoplus \operatorname{Ker} b(t) d \mu(t) \neq\{\mathbf{0}\}
$$


It is easy to see that

$$
\{\mathbf{0}\} \neq \int \bigoplus \operatorname{Ker} b(t) d \mu(t) \subset \operatorname{Ker} B .
$$

However, it is obvious (and well known) that $\operatorname{Ker}\left(S^{*}-S\right)=\{\mathbf{0}\}$.

Thus $b(t)$ is an injective map from $\widetilde{\mathcal{H}}(t)$ to $\mathcal{H}(-t)$ which implies that

$$
\nu(-t)=\operatorname{dim} \mathcal{H}(-t) \geqslant \operatorname{dim} \tilde{\mathcal{H}}(t) \geqslant \nu(t)-2, \quad \mu \text {-a.e. }
$$

Interchanging the roles of $t$ and $-t$, we obtain

$$
|\nu(t)-\nu(-t)| \leqslant 2
$$

Let us now prove that $|\nu(t)-\nu(-t)| \leqslant 1$ on the singular spectrum. Let $\Delta_{s}$ be a Borel set such that $\mu_{s}$ is supported on $\Delta_{s}$ and $\Delta_{s}$ has zero Lebesgue measure.

LEMMA 1.2. Let $f$ be a function supported on $\Delta_{s}$ such that for $\mu$-almost all $t$, $f(t) \perp e_{0}(t)$, and if $e_{0}(t)=0$, then $f(t) \perp(S \alpha)(t)$. Then $\Gamma S^{*} f=S \Gamma f$.

Let us first complete the proof of Theorem 1.1 and then prove Lemma 1.2. Put

$$
\check{\mathcal{H}}(t)=\left\{x \in \mathcal{H}(t): x \perp e_{0}(t) \text { and if } e_{0}(t)=0 \text {, then } x \perp(S \alpha)(t)\right\}, \quad t \in \Delta_{s}
$$

and

$$
\check{\mathcal{H}}=\left\{f: f \text { is supported on } \Delta_{s} \text { and } f(t) \in \check{\mathcal{H}}(t), \mu \text {-a.e. }\right\} \text {. }
$$

As above we put $A_{1}=A \mid \check{\mathcal{H}}, A_{2}=-A, B=S^{*}-S$. By Lemma $1.2, B A_{1}=A_{2} B$ and by Lemma 3 ,

$$
(B f)(t)=b(t) f(-t)
$$

where $b$ is a weakly measurable operator-valued function on $\Delta_{s}$ such that $b(t): \dot{\mathcal{H}}(t) \rightarrow$ $\mathcal{H}(-t)$ and $\operatorname{Ker} b(t)=\{\boldsymbol{0}\}, \mu$-a.e. As above it follows that

$$
\nu(-t)=\operatorname{dim} \mathcal{H}(-t) \geqslant \operatorname{dim} \check{\mathcal{H}}(t) \geqslant \nu(t)-1
$$

which implies that

$$
|\nu(t)-\nu(-t)| \leqslant 1, \quad \mu_{s} \text {-a.e. }
$$

Proof of Lemma 1.2. Put

$$
\Delta_{0}=\left\{t \in \Delta_{s}: e_{0}(t) \neq 0\right\}
$$

(recall that $\left\{e_{j}\right\}_{j \geqslant 0}$ is an orthonormal basis in which $\Gamma$ has Hankel matrix). If $f$ is supported on $\Delta_{s} \backslash \Delta_{0}$, then $f \perp S \alpha$ and $f \perp e_{0}$, and it follows from (1.4) that $\Gamma S^{*} f=S \Gamma f$. 
Thus we can assume without loss of generality that $f$ is supported on $\Delta_{0}$. Let $k$ be a positive integer. Put

$$
\Delta_{k}=\left\{t \in \Delta_{s}: 2^{-k} \leqslant\left\|e_{0}(t)\right\| \leqslant 2^{k}\right\} .
$$

Clearly $\Delta_{0}=\bigcup_{k \geqslant 1} \Delta_{k}$. So it is sufficient to consider the case when $f$ is supported on $\Delta_{k}$ for some $k$.

Let $\left\{I_{j}\right\}$ be a covering of $\Delta_{k}$ by disjoint open intervals. Put $\Lambda_{j}=\Delta_{k} \cap I_{j}$ ( $k$ is fixed), $f_{j}=\chi_{j} f, g_{j}=\chi_{j} e_{0}$, where $\chi_{j}$ is the characteristic function of $\Lambda_{j}$.

It follows from (1.3) and (1.4) that

$$
\Gamma\left(S+S^{*}\right) f-\left(S+S^{*}\right) \Gamma f=c e_{0}
$$

for some $c \in \mathbf{C}$. Therefore it is sufficient to show that

$$
\left(\Gamma\left(S+S^{*}\right) f-\left(S+S^{*}\right) \Gamma f, e_{0}\right)=0 .
$$

We have

$$
\begin{aligned}
\left(\Gamma\left(S+S^{*}\right) f-\left(S+S^{*}\right) \Gamma f, e_{0}\right) & =\sum_{j \geqslant 1}\left(\Gamma\left(S+S^{*}\right) f_{j}-\left(S+S^{*}\right) \Gamma f_{j}, e_{0}\right) \\
& =\sum_{j \geqslant 1} \frac{1}{\left(e_{0}, g_{j}\right)}\left(\Gamma\left(S+S^{*}\right) f_{j}-\left(S+S^{*}\right) \Gamma f_{j}, g_{j}\right)
\end{aligned}
$$

in view of (1.6).

Clearly

$$
\left(e_{0}, g_{j}\right)=\int_{\Lambda_{j}}\left\|e_{0}(t)\right\|^{2} d \mu(t) \geqslant 2^{-2 k} \mu \Lambda_{j}
$$

Let $\lambda \in \Lambda_{j}$. We have

$$
\left|\left(\Gamma\left(S+S^{*}\right) f-\left(S+S^{*}\right) \Gamma f, e_{0}\right)\right| \leqslant 2^{2 k} \sum_{j \geqslant 1} \frac{1}{\mu \Lambda_{j}}\left|\left(\left(S+S^{*}\right) f_{j}, \Gamma g_{j}\right)-\left(\Gamma f_{j},\left(S+S^{*}\right) g_{j}\right)\right|
$$

Put $g^{(j)}=\Gamma g_{j}-\lambda_{j} g_{j}, f^{(j)}=\Gamma f_{j}-\lambda_{j} f_{j}$. Clearly

$$
\left\|g^{(j)}\right\| \leqslant\left|I_{j}\right| \cdot\left\|g_{j}\right\|, \quad\left\|f^{(j)}\right\| \leqslant\left|I_{j}\right| \cdot\left\|f_{j}\right\|,
$$

where $\left|I_{j}\right|$ is the length of $I_{j}$. Therefore

$$
\begin{aligned}
\left|\left(\Gamma\left(S+S^{*}\right) f-\left(S+S^{*}\right) \Gamma f, e_{0}\right)\right| & \leqslant 2^{2 k} \sum_{j \geqslant 1} \frac{1}{\mu \Lambda_{j}}\left|\left(\left(S+S^{*}\right) f_{j}, g^{(j)}\right)-\left(f^{(j)},\left(S+S^{*}\right) g_{j}\right)\right| \\
& \leqslant \text { const } \cdot \sum_{j \geqslant 1} \frac{\left|I_{j}\right|}{\mu \Lambda_{j}}\left\|f_{j}\right\| \cdot\left\|g_{j}\right\| \\
& \leqslant \text { const } \cdot \sup _{j \geqslant 1} \frac{\left|I_{j}\right|}{\mu \Lambda_{j}} \cdot\left(\sum_{j \geqslant 1}\left\|f_{j}\right\|^{2}\right)^{1 / 2}\left(\sum_{j \geqslant 1}\left\|g_{j}\right\|^{2}\right)^{1 / 2} \\
& =\text { const } \cdot \sup _{j \geqslant 1} \frac{\left|I_{j}\right|}{\mu \Lambda_{j}} \cdot\|f\| \cdot\left\|e_{0}\right\| .
\end{aligned}
$$


Clearly the proof will be completed if we establish the following elementary fact.

Lemma 1.3. Let $\mu$ be a singular measure supported on a Borel set $\Omega$ of zero Lebesgue measure and let $\varepsilon>0$. Then $\Omega$ can be covered by disjoint open intervals $\left\{I_{j}\right\}_{j} \geqslant 1$ such that

$$
\sup _{j \geqslant 1} \frac{\left|I_{j}\right|}{\mu\left(\Omega \cap I_{j}\right)} \leqslant \varepsilon
$$

Proof of Lemma 1.3. Let $\left\{J_{k}\right\}_{k \leqslant 1}$ be a covering of $\Omega$ by disjoint open intervals such that $\sum_{k \geqslant 1}\left|J_{k}\right|<\frac{1}{2} \varepsilon \mu \Omega$. Let

$$
M_{1}=\left\{k: \frac{\left|J_{k}\right|}{\mu\left(\Omega \cap J_{k}\right)}>\varepsilon\right\}
$$

Then

$$
\mu\left(\bigcup_{k \in M_{1}}\left(\Omega \cap J_{k}\right)\right)<\frac{1}{\varepsilon} \sum_{k \in M_{1}}\left|J_{k}\right|<\frac{1}{2} \mu \Omega .
$$

Let $\Omega^{(1)}=\bigcup_{k \in M_{1}}\left(\Omega \cap J_{k}\right)$. Let us cover $\Omega^{(1)}$ by a system of disjoint open intervals the sum of lengths of which is less than $\frac{1}{2} \varepsilon \mu \Omega^{(1)}$. Then the intervals $J$ from this system for which

$$
\frac{|J|}{\mu\left(\Omega^{(1)} \cap J\right)}<\varepsilon
$$

cover a subset of $\Omega^{(1)}$ of measure greater than $\frac{1}{2} \mu \Omega^{(1)}$. By repeating this procedure, we can construct a covering of $\Omega$ by disjoint intervals $\left\{I_{j}\right\}_{j \geqslant 1}$ satisfying (1.7).

\section{I.2. Eigenvalues of Hankel operators}

As we have already noticed, it follows from Theorem 1.1 that for any self-adjoint Hankel operator $\Gamma$ and for any $\lambda \in \mathbf{R}$

$$
|\operatorname{dim} \operatorname{Ker}(\Gamma-\lambda I)-\operatorname{dim} \operatorname{Ker}(\Gamma+\lambda I)| \leqslant 1 .
$$

The main result of this section shows that (2.1) is also valid for an arbitrary bounded Hankel operator and an arbitrary $\lambda \in \mathbf{C}$. The idea of the proof is the same as in Theorem 1.1, but to include the non-self-adjoint case, we have to replace the inner product by the natural bilinear form on $l^{2}$ and instead of adjoint operators consider transposed operators.

We assume here that $\Gamma$ is a bounded Hankel operator on the Hilbert space $l^{2},\left\{e_{n}\right\}$ is the standard orthonormal basis in $l^{2}$. Then $\left(\Gamma e_{j}, e_{k}\right)=\alpha_{j+k}, j, k \geqslant 0$, where $\left\{\alpha_{j}\right\}_{j \geqslant 0}$ is a sequence of complex numbers. 
It is convenient to consider the following bilinear form on $l^{2}$ :

$$
\langle x, y\rangle=\sum_{j \geqslant 0} x_{j} y_{j}
$$

where $x=\sum_{j \geqslant 0} x_{j} e_{j}$ and $y=\sum_{j \geqslant 0} y_{j} e_{j}$. If $B$ is a bounded operator on $l^{2}$, we define its transpose $B^{t}$ by

$$
\langle B x, y\rangle=\left\langle x, B^{t} y\right\rangle, \quad x, y \in l^{2} .
$$

Clearly any Hankel operator $\Gamma$ is symmetric: $\Gamma^{t}=\Gamma$. Let $S$ be the shift operator on $l^{2}$ : $S e_{n}=e_{n+1}, n \geqslant 0$. Then $S^{*}=S^{t}$.

THEOREM 2.1. Let $\Gamma$ be a bounded Hankel operator. Then inequality (2.1) holds for any $\lambda \in \mathbf{C}$.

Proof. Given $\mu \in \mathbf{C}$, we put $E_{\mu}=\operatorname{Ker}(\Gamma-\mu I)$. Let $\alpha=\sum_{j \geqslant 0} \alpha_{j} e_{j}$. We have

$$
\begin{gathered}
S^{*} \Gamma=\Gamma S, \\
S \Gamma x-\Gamma S^{*} x=\left\langle x, e_{0}\right\rangle S \alpha-\langle x, S \alpha\rangle e_{0}, \quad x \in l^{2},
\end{gathered}
$$

(see (1.3) and (1.4)).

Let $\lambda \in \mathbf{C}$. To prove the theorem, we have to show that

$$
\operatorname{dim} E_{-\lambda} \geqslant \operatorname{dim} E_{\lambda}-1
$$

We consider separately two different cases.

Case 1. There exists a vector $a=\sum_{j \geqslant 0} a_{j} e_{j}$ in $E_{\lambda}$ with $a_{0} \neq 0$. Let us show that if $x \in E_{\lambda}$ and $\left\langle x, e_{0}\right\rangle=0$, then

$$
S \Gamma x=\Gamma S^{*} x,
$$

which is equivalent in view of $(2.2)$ to

$$
\Gamma\left(S+S^{*}\right) x=\left(S+S^{*}\right) \Gamma x .
$$

It follows from (2.2) and (2.3) that

$$
\Gamma\left(S+S^{*}\right) x-\left(S+S^{*}\right) \Gamma x=c e_{0}
$$

for some $c \in \mathbf{C}$. Since $\left\langle a, e_{0}\right\rangle \neq 0$ and

$$
\left\langle\Gamma\left(S+S^{*}\right) x-\left(S+S^{*}\right) \Gamma x, a\right\rangle=a_{0} c,
$$


it is sufficient to show that

$$
\left\langle\Gamma\left(S+S^{*}\right) x-\left(S+S^{*}\right) \Gamma x, a\right\rangle=0
$$

We have

$$
\begin{aligned}
\left\langle\Gamma\left(S+S^{*}\right) x-\left(S+S^{*}\right) \Gamma x, a\right\rangle & =\left\langle\left(S+S^{*}\right) x, \Gamma a\right\rangle-\left\langle\left(S+S^{*}\right) \Gamma x, a\right\rangle \\
& =\lambda\left\langle\left(S+S^{*}\right) x, a\right\rangle-\lambda\left\langle\left(S+S^{*}\right) x, a\right\rangle
\end{aligned}
$$

since both $x$ and $a$ belong to $E_{\lambda}$.

Now it is easy to show that if $x \in E_{\lambda}$ and $\langle x, S \alpha\rangle=0$, then $\left(S-S^{*}\right) x \in E_{-\lambda}$. Indeed

$$
\Gamma\left(S-S^{*}\right) x=\Gamma S x-\Gamma S^{*} x=S^{*} \Gamma x-S \Gamma x=-\left(S-S^{*}\right) \Gamma x=-\lambda\left(S-S^{*}\right) x
$$

by (2.2) and (2.5). Since $\operatorname{Ker}\left(S-S^{*}\right)=\{0\}$, it follows that $S-S^{*}$ is a one-to-one map of $\left\{x \in E_{\lambda}:\langle x, S \alpha\rangle=0\right\}$ into $E_{-\lambda}$ which proves (2.4).

Case 2. For any $x \in E_{\lambda},\left\langle x, e_{0}\right\rangle=0$. In this case it follows directly from (2.2) and (2.3) that if $x \in E_{\lambda}$ and $\langle x, S \alpha\rangle=0$, then (2.5) holds. As in Case 1 this implies that $S-S^{*}$ is a one-to-one map of $\left\{x \in E_{\lambda}:\langle x, S \alpha\rangle=0\right\}$ into $E_{-\lambda}$.

\section{Balanced realizations with continuous time}

In this chapter we use linear systems with continuous time to construct a Hankel operator with prescribed spectral properties. Recall that such linear systems allow one to solve the problem of the description of non-negative operators that are unitarily equivalent to the modulus of a Hankel operator [T2]. In this chapter we construct self-adjoint Hankel operators with prescribed spectral properties in the case when $|\nu(t)-\nu(-t)| \leqslant 1$ almost everywhere. Moreover we shall find an explicit formula for the symbol of the Hankel operator.

However we shall see that this approach cannot lead to a complete solution of the problem of the description of self-adjoint operators that are unitarily equivalent to a Hankel operator. Namely, if $\Gamma$ is a positive operator with spectral multiplicity 2, it cannot be obtained from a linear system that involves bounded operators.

In §II.1 we introduce the balanced systems, define the Hankel operator associated with the system and state the main result of the chapter. In $§ I I .2$ we study the Hankel operator associated with a balanced system and describe its spectral type modulo the kernel. In $§ I I .3$ we use Lyapunov's equation to reduce the problem in question to the 
construction of an asymptotically stable semigroup whose generator satisfies certain commutation relations. The main construction is given in $\S I I .4$ which allows us to solve the problem modulo the kernel. The main result of the chapter is proved in $\S I I .5$ where we study the kernel of the Hankel operator associated with a balanced system. In §II.6 we prove auxiliary facts in measure theory. In the final $\S I I .7$ we show that positive operators with multiple spectrum cannot be constructed with the help of a balanced system with continuous time.

\section{II.1. Hankel operators and balanced realizations}

We consider here linear systems $\{A, B, C\}$ with one-dimensional input and one-dimensional output:

$$
\left\{\begin{array}{l}
x^{\prime}(t)=A x(t)+B u(t), \\
y(t)=C x(t) .
\end{array}\right.
$$

Here $A$ is a bounded linear operator on a Hilbert space $\mathcal{K}$ (state space), $B: \mathbf{C} \rightarrow \mathcal{K}$ and $C: \mathcal{K} \rightarrow \mathbf{C}$ are bounded linear operators defined by

$$
B u=u b, u \in \mathbf{C}, \quad C x=(x, c), x \in \mathcal{K},
$$

where $c, b \in \mathcal{K}$. In (1.1) $u$ is interpreted as input and $y$ as output.

In what follows we shall also denote the system $(1.1)$ by $\{A, b, c\}$.

The system (1.1) is controllable if $\operatorname{span}\left\{A^{n} b: n \geqslant 0\right\}=\mathcal{K}$ and observable if $\operatorname{span}\left\{A^{* n} c\right.$ : $n \geqslant 0\}=\mathcal{K}$. It is called minimal if it is both controllable and observable.

We can associate with the system (1.1) the controllability Gramian $W_{c}$ and the observability Gramian $W_{o}$ defined by

$$
\begin{aligned}
& W_{c} \stackrel{\text { def }}{=} \int_{\mathbf{R}_{+}} e^{t A} B B^{*} e^{t A^{*}} d t, \\
& W_{o} \stackrel{\text { def }}{=} \int_{\mathbf{R}_{+}} e^{t A^{*}} C^{*} C e^{t A} d t,
\end{aligned}
$$

if the integrals converge in the weak operator topology. It is easy to see that if the integrals converge, then the system is controllable (observable) if and only if $\operatorname{Ker} W_{c}=\{0\}$ $\left(\operatorname{Ker} W_{o}=\{0\}\right)$.

In what follows, an important role will be played by balanced linear systems. A minimal system is called balanced (cf. [G], where the case $\operatorname{dim} \mathcal{K}<\infty$ was considered) if the integrals (1.2) and (1.3) converge in the weak operator topology and $W_{c}=W_{o}$.

We can associate with the system $\{A, B, C\}$ the following formal Hankel operator $\Gamma_{h}$, where

$$
h(t)=C e^{t A} B=\left(e^{t A} b, c\right), \quad t>0,
$$


which is defined on compactly supported functions by

$$
\left(\Gamma_{h} f\right)(s)=\int_{0}^{\infty} h(t+s) f(t) d t .
$$

In general $\Gamma_{h}$ need not be bounded on $L^{2}\left(\mathbf{R}_{+}\right)$. However we shall see later that for the balanced linear systems the operator $\Gamma_{h}$ is bounded.

The operator $\Gamma_{h}$ is related to the system (1.1) in a natural way in the case $\sup _{t>0}\left\|e^{t A}\right\|<\infty$. Namely, let $v \in L^{2}\left(\mathbf{R}_{+}\right)$. Consider the function $u$ on $\mathbf{R}$ defined by

$$
u(t)= \begin{cases}v(-t), & t<0, \\ 0, & t \geqslant 0 .\end{cases}
$$

It is well known (and it is easy to see) that under the initial condition $\lim _{t \rightarrow-\infty} x(t)=\mathbf{0}$ the output $y$ of the system (1.1) with input $u$ satisfies

$$
y(t)=\left(\Gamma_{h} v\right)(t), \quad t \geqslant 0 .
$$

Now we are in a position to formulate the main result of Chapter II. Let $\Gamma$ be a selfadjoint operator. Then $\Gamma$ is unitarily equivalent to multiplication by the independent variable on the direct integral

$$
\int \bigoplus \mathcal{H}(t) d \mu(t)
$$

Let $\nu(t) \stackrel{\text { def }}{=} \operatorname{dim} \mathcal{H}(t)$ be the multiplicity function of $\Gamma$.

THEOREM 1.1. Let $\Gamma$ be a self-adjoint operator such that

(C1) either $\operatorname{Ker} \Gamma=\{0\}$ or $\operatorname{dim} \operatorname{Ker} \Gamma=\infty$;

(C2) $\Gamma$ is non-invertible;

$\left(\mathrm{C} 3^{\prime}\right)|\nu(t)-\nu(-t)| \leqslant 1, \mu-a . e$.

Then there exists a balanced system $\{A, B, C\}$ such that the corresponding Hankel operator $\Gamma_{h}$ is unitarily equivalent to $\Gamma$.

The proof of Theorem 1.1 will be given in $\S I I .5$.

\section{II.2. Unitary equivalence modulo the kernel}

In this section we establish useful facts on the unitary equivalence (modulo the kernel) of the Hankel operator $\Gamma_{h}$ corresponding to a balanced system $\{A, B, C\}$ and certain operators related to the system.

The following theorem was proved in $[\mathrm{G}]$ in the case $\operatorname{dim} \mathcal{K}<\infty$ and [T2] in the general case. We prove it here for completeness. We also need the construction used in the proof. 
THEOREM 2.1. Let $\{A, B, C\}$ be a balanced system, $W=W_{o}=W_{c}$, and let $\Gamma_{h}$ be the Hankel operator associated with it. Then $\Gamma_{h}$ is bounded and the restriction of $\left|\Gamma_{h}\right|$ to $\left(\operatorname{Ker} \Gamma_{h}\right)^{\perp}$ is unitarily equivalent to $W$.

Proof. Consider the operators $V_{c}, V_{o}: \mathcal{K} \rightarrow L^{2}\left(\mathbf{R}_{+}\right)$defined by

$$
\left(V_{c} x\right)(t)=B^{*} e^{t A^{*}} x, \quad\left(V_{o} x\right)(t)=c e^{t A} x, \quad x \in \mathcal{H}
$$

Clearly

$$
V_{c}^{*} V_{c}=\int_{\mathbf{R}_{+}} e^{t A} B B^{*} e^{t A^{*}} d t=W_{c}=W
$$

and

$$
V_{o}^{*} V_{o}=\int_{\mathbf{R}_{+}} e^{t A^{*}} C^{*} C e^{t A} d t=W_{o}=W .
$$

Therefore the operators $V_{c}$ and $V_{o}$ are bounded. It is also easy to see that

$$
\Gamma_{h}=V_{o} V_{c}^{*}
$$

which implies that $\Gamma_{h}$ is also bounded.

Let

$$
V_{c}=U_{c} W^{1 / 2}, \quad V_{o}=U_{o} W^{1 / 2}
$$

be the polar decompositions of $V_{c}$ and $V_{o}\left(\left|V_{c}\right|=\left|V_{o}\right|=W^{1 / 2}\right)$. We have

$$
\operatorname{Ker} V_{c}=\operatorname{Ker} V_{c}^{*} V_{c}=\operatorname{Ker} W_{c}=\{\mathbf{0}\}=\operatorname{Ker} W_{o}=\operatorname{Ker} V_{o}^{*} V_{o}=\operatorname{Ker} V_{o}
$$

Therefore the operators $U_{c}$ and $U_{o}$ are isometries. Clearly Range $U_{c}=$ clos Range $V_{c}$ and Range $U_{o}=$ clos Range $V_{o}$. It follows that

$$
\left|\Gamma_{h}\right|^{2}=\Gamma_{h}^{*} \Gamma_{h}=V_{c} V_{o}^{*} V_{o} V_{c}^{*}=U_{c} W^{2} U_{c}^{*}
$$

Since $\operatorname{Ker} U_{c}^{*}=\left(\operatorname{Range} V_{c}\right)^{\perp}=\operatorname{Ker} V_{c}^{*}=\operatorname{Ker} \Gamma_{h}$, the operator $W^{2}$ is unitarily equivalent to $\left|\Gamma_{h}\right|^{2} \mid\left(\operatorname{Ker} \Gamma_{h}\right)^{\perp}$ which implies that $\left|\Gamma_{h}\right| \mid\left(\operatorname{Ker} \Gamma_{h}\right)^{\perp}$ is unitarily equivalent to $W$.

Let us consider now linear systems for which the corresponding Hankel operator is self-adjoint.

THEOREM 2.2. Let $\{A, B, C\}$ be a balanced linear system (1.1) such that $\Gamma_{h}$ is selfadjoint, $W=W_{o}=W_{c}$. Then there exists an operator $J$ on $\mathcal{K}$ which is self-adjoint and unitary and such that $\boldsymbol{J} W=W \boldsymbol{J}, A^{*}=\boldsymbol{J} A \boldsymbol{J}$, and $c=\boldsymbol{J} b$.

Proof. We have under the above notation $\Gamma_{h}=U_{o} W U_{c}^{*}$. Since $\Gamma_{h}$ is self-adjoint, we have

$$
U_{o} W U_{c}^{*}=U_{c} W U_{o}^{*}
$$


hence

$$
W U=U^{*} W
$$

where $U=U_{c}^{*} U_{o}$.

We have already shown that $\operatorname{Ker} U_{c}^{*}=\operatorname{Ker} V_{c}^{*}=\operatorname{Ker} \Gamma_{h}$. Since $\Gamma_{h}^{*}=\Gamma_{h}$, it follows that $\Gamma_{h}=V_{c} V_{o}^{*}$ which implies that $\operatorname{Ker} \Gamma_{h}=\operatorname{Ker} V_{o}^{*}=\operatorname{Ker} U_{o}^{*}$. Therefore $U_{c}$ and $U_{o}$ are isometric with the same range $\left(\operatorname{Ker} \Gamma_{h}\right)^{\perp}$ which implies that $U=U_{c}^{*} U_{o}$ is unitary. Let us show that it is also self-adjoint.

LEMMA 2.3. Let $W$ be a non-negative operator, $\operatorname{Ker} W=\{0\}$ and let $U$ be a unitary operator such that $W U=U^{*} W$. Then $U$ is self-adjoint.

Proof. $\left({ }^{4}\right)$ Multiplying the equality $U^{*} W=W U$ by $U$ on the left and by $U^{*}$ on the right, we obtain the equality $U W=W U^{*}$. Hence

$$
U^{*} W^{2} U=W U W U=W^{2} U^{*} U=W^{2}
$$

which implies that $W^{2} U=U W^{2}$. Since $W$ is positive, it follows that $W U=U W$ and so

$$
U^{*} W=W U=U W,
$$

and since $W$ has dense range, we have $U^{*}=U$.

Let us complete the proof of Theorem 2.2. We denote $U=U_{c}^{*} U_{o}$ by $\boldsymbol{J}$. It follows from (2.1) that $W J=J W$. Let us show that $A^{*}=J A J$. Indeed by the definition of $J$, $U_{o}=U_{c} \boldsymbol{J}$ and since $\boldsymbol{J} W=W \boldsymbol{J}$, we have $V_{o}=V_{c} \boldsymbol{J}$. This means that

$$
C e^{t A} x=B e^{t A^{*}} J x, \quad x \in \mathcal{K}
$$

or which is the same

$$
\left(x, e^{t A^{*}} c\right)=\left(x, \boldsymbol{J} e^{t A} b\right)=\left(x, \boldsymbol{J} e^{t A} \boldsymbol{J}(\boldsymbol{J} b)\right)=\left(x, e^{t J A J} J b\right) .
$$

Hence

$$
e^{t A^{*}} c=e^{t \boldsymbol{J} A J} J b, \quad t \geqslant 0 .
$$

Substituting $t=0$, we obtain $c=\boldsymbol{J} b$. Differentiating (2.2) we find that $A^{*}$ and $\boldsymbol{J} A \boldsymbol{J}$ coincide on the orbit $\left\{e^{t A^{*}} c: t \geqslant 0\right\}$ which is dense since the system is observable. Therefore $A^{*}=\boldsymbol{J} A \boldsymbol{J}$ which completes the proof.

$\left.{ }^{4}\right)$ This proof was suggested by the referee and is simpler than the original proof suggested by the authors.

18-950233 Acta Mathematica 174. Imprimé le 20 juin 1995 
THEOREM 2.4. Let $\{A, B, C\}$ be a balanced dynamical system (1.1), $W_{c}=W_{o}=W$, and let $\boldsymbol{J}$ be an operator which is self-adjoint and unitary and satisfies the equalities $\boldsymbol{J} W=W \boldsymbol{J}, A^{*}=\boldsymbol{J} A \boldsymbol{J}, c=\boldsymbol{J} b$. Then the Hankel operator $\Gamma_{h}$ associated with the system is self-adjoint and $\Gamma_{h} \mid\left(\operatorname{Ker} \Gamma_{h}\right)^{\perp}$ is unitarily equivalent to WJ.

Proof. Since $A^{*}=\boldsymbol{J} A \boldsymbol{J}$, we have $e^{t A^{*}}=\boldsymbol{J} e^{t A} \boldsymbol{J}$. The equality $c=\boldsymbol{J} b$ implies $C=B^{*} \boldsymbol{J}$ which in turn leads to the equality $V_{c}=V_{o} J$. Therefore

$$
\Gamma_{h}=V_{o} \boldsymbol{J} V_{o}^{*}=U_{o} W^{1 / 2} J W^{1 / 2} U_{o}^{*}=U_{o} W J U_{o}^{*}
$$

which proves the result.

\section{II.3. Lyapunov equations}

We are going to use Theorem 2.4 to construct a Hankel operator with given spectral properties modulo the kernel. Namely let $\Gamma$ be the self-adjoint operator in Theorem 1.1. Put $\widetilde{\Gamma}=\Gamma \mid(\operatorname{Ker} \Gamma)^{\perp}$. Let $J$ be the operator on $(\operatorname{Ker} \Gamma)^{\perp}$ which is self-adjoint and unitary and satisfies $\widetilde{\Gamma}=\boldsymbol{J}|\widetilde{\Gamma}|=|\widetilde{\Gamma}| \boldsymbol{J}$. We are going to construct a balanced linear system $\{A, B, C\}$ such that $W=|\widetilde{\Gamma}|, A^{*}=\boldsymbol{J} A \boldsymbol{J}, c=\boldsymbol{J} b$, and $W \boldsymbol{J}=\boldsymbol{J} W$. Then by Theorem $2.4, \Gamma_{h} \mid\left(\operatorname{Ker} \Gamma_{h}\right)^{\perp}$ is unitarily equivalent to $\widetilde{\Gamma}$. Later we shall settle the problem with the kernel.

It is not easy to verify directly that $W=|\widetilde{\Gamma}|$. Fortunately, to prove this equality, we do not have to evaluate the integrals (1.2) and (1.3). We are going to verify instead the corresponding Lyapunov equations.

Recall that an operator $A$ is said to be asymptotically stable if

$$
\lim _{t \rightarrow \infty}\left\|e^{t A} x\right\|=0
$$

for any $x$.

THEOREM 3.1. Let $A$ be an asymptotically stable operator on a Hilbert space $\mathcal{K}$ and let $K$ be a bounded operator on $\mathcal{K}$. If the integral

$$
\int_{\mathbf{R}_{+}} e^{t A^{*}} K e^{t A} d t \stackrel{\text { def }}{=} W
$$

converges in the weak operator topology, then $W$ is a unique solution of the following Lyapunov equation

$$
A^{*} W+W A=-K
$$


Proof. Let us show that $W$ satisfies (3.2). We have for $x, y \in \mathcal{K}$

$$
\begin{aligned}
\left(\left(A^{*} W+W A\right) x, y\right) & =\int_{\mathbf{R}_{+}}\left(\left(A^{*} e^{t A^{*}} K e^{t A}+e^{t A^{*}} K e^{t A} A\right) x, y\right) d t \\
& =\int_{\mathbf{R}_{+}}\left(\frac{d}{d t}\left(e^{t A^{*}} K e^{t A} x, y\right)\right) d t \\
& =\lim _{t \rightarrow \infty}\left(K e^{t A} x, e^{t A} y\right)-(K x, y)=-(K x, y)
\end{aligned}
$$

because of the asymptotic stability of $A$.

Let us now establish the uniqueness of the solution. Suppose

$$
A^{*} X+X A=-K
$$

for some operator $X$. Let $\Delta=W-X$. Then

$$
A^{*} \Delta+\Delta A=\mathbf{0}
$$

Clearly

$$
\frac{d}{d t}\left(\Delta e^{t A} x, e^{t A} y\right)=\left(\Delta A e^{t A} x, e^{t A} y\right)+\left(\Delta e^{t A} x, A e^{t A} y\right)=\left(\left(A^{*} \Delta+\Delta A\right) e^{t A} x, y\right)=0 .
$$

Since $\lim _{t \rightarrow \infty}\left\|e^{t A} x\right\|=0$ for any $x \in \mathcal{K}$, it follows that $(\Delta x, y)=0$ for any $x, y \in \mathcal{K}$. Hence $\Delta=\mathbf{0}$.

The following result shows that if $W$ satisfies the Lyapunov equation (2.2), we can obtain the convergence of the integral (3.1) for free.

TheOREM 3.2. Let $A$ be an operator such that $\left\|e^{t A}\right\| \leqslant M<\infty, t \geqslant 0$, and let $K$ be a non-negative operator. If $W$ is a solution of the Lyapunov equation

$$
A^{*} W+W A=-K
$$

then the integral

$$
\int_{\mathbf{R}_{+}} e^{t A^{*}} K e^{t A} d t
$$

converges in the weak operator topology.

Proof. Let $x, y \in \mathcal{K}$. We have

$$
\left(e^{t A^{*}} K e^{t A} x, y\right)=-\left(e^{t A^{*}}\left(A^{*} W+W A\right) e^{t A} x, y\right)=-\frac{d}{d t}\left(e^{t A^{*}} W e^{t A} x, y\right) .
$$


Therefore

$$
\int_{0}^{T}\left(e^{t A^{*}} K e^{t A} x, x\right) d t=(W x, x)-\left(e^{T A^{*}} W e^{T A} x, x\right) \leqslant\|W\| \cdot(M+1)
$$

Since $\left(e^{t A^{*}} K e^{t A} x, x\right) \geqslant 0$ for $x \in \mathcal{K}$, it follows that the integral $\int_{0}^{\infty}\left(e^{t A^{*}} K e^{t A} x, x\right) d t$ converges for any $x \in \mathcal{K}$. The result follows from the polarization identity.

Theorems 3.1 and 3.2 show that to solve the problem modulo the kernel, it is sufficient to construct a linear system $\{A, B, C\}$ and an operator $\boldsymbol{J}=\boldsymbol{J}^{*}=\boldsymbol{J}^{-1}$ such that

(i) the operators $A$ and $A^{*}$ are asymptotically stable;

(ii) the operator $W=|\widetilde{\Gamma}|$ is a solution of the Lyapunov equations

$$
A^{*} W+W A=-C^{*} C, \quad A W+W A^{*}=-B B^{*}
$$

(iii) $A^{*}=\boldsymbol{J} A \boldsymbol{J}, c=\boldsymbol{J} b$.

If $A^{*}=\boldsymbol{J} A \boldsymbol{J}$, then $A^{*}$ is asymptotically stable if and only if $A$ is. It follows easily from (iii) that both Lyapunov equations in (ii) coincide. Therefore it is sufficient to verify the following properties:

(i') $A$ is asymptotically stable;

(ii') $A^{*} W+W A=-C^{*} C$;

(iii') $A^{*}=\boldsymbol{J} A \boldsymbol{J}, \boldsymbol{c}=\boldsymbol{J} b$.

\section{II.4. The main construction}

Let $\Gamma$ be a self-adjoint operator which satisfies the hypotheses of Theorem 1.1 and let $\widetilde{\Gamma}=\Gamma \mid(\operatorname{Ker} \Gamma)^{\perp}$. Then the operator $|\widetilde{\Gamma}|$ is unitarily equivalent to the operator $W$ of multiplication by $t$ on the von Neumann integral

$$
\mathcal{K}=\int_{\sigma(W)} \oplus E(t) d \varrho(t)
$$

Here $\varrho$ is a scalar spectral measure of $|\widetilde{\Gamma}|$. We can also assume that the spaces $E(t)$ are imbedded in an infinite-dimensional space $E$ with an orthonormal basis $\left\{e_{j}\right\}_{j \geqslant 1}$ and

$$
E(t)=\operatorname{span}\left\{e_{k}: 1 \leqslant k<\nu_{W}(t)+1\right\}, \quad E(t)=\{0\} \text { if } \nu_{W}(t)=0,
$$

where $\nu_{W}$ is the spectral multiplicity function of $W, \nu_{W}(t)=\operatorname{dim} E(t)$.

Clearly $\nu_{W}(t)=\nu(t)+\nu(-t), \varrho$-a.e., where $\nu$ is the spectral multiplicity function of $\Gamma$. Recall that $\nu$ satisfies the condition $\left(\mathrm{C}^{\prime}\right)$ of Theorem 1.1. 
Consider the sets

$$
\begin{aligned}
& \sigma_{+}=\{t \in \sigma(W): \nu(t) \geqslant \nu(-t)\} \\
& \sigma_{-}=\{t \in \sigma(W): \nu(t)<\nu(-t)\}
\end{aligned}
$$

We define the operator $J$ on $\mathcal{K}$ as multiplication by the operator-valued function $J$ :

$$
J(t)=\xi(t)\left(\begin{array}{cccc}
1 & 0 & 0 & \ldots \\
0 & -1 & 0 & \ldots \\
0 & 0 & 1 & \ldots \\
\vdots & \vdots & \vdots & \ddots
\end{array}\right)
$$

where $\xi(t)=1$ for $t \in \sigma_{+}$and $\xi(t)=-1$ for $t \in \sigma_{-}$.

Clearly $\widetilde{\Gamma}$ is unitarily equivalent to $\boldsymbol{J} W$ and we can assume that $\widetilde{\Gamma}=\boldsymbol{J} W$.

Recall that the scalar spectral measure $\varrho$ of $W$ is not uniquely defined and we can always replace it with a mutually absolutely continuous measure by multiplying it by a positive weight $w$ in $L^{1}(\varrho)$

Let $\mathcal{K}_{0}$ be the subspace of $\mathcal{K}$ that consists of functions $f$ of the form

$$
f(t)=\varphi(t) e_{1}, \quad \varphi \in L^{2}(\varrho) .
$$

$\mathcal{K}_{0}$ can be identified naturally with $L^{2}(\varrho)$. Let $A_{0}$ be the integral operator on $\mathcal{K}_{0}=L^{2}(\varrho)$ defined by

$$
\left(A_{0} f\right)(s)=\int_{\sigma(W)} k(s, t) f(t) d \varrho(t)
$$

where

$$
k(s, t)= \begin{cases}-1 /(s+t), & s, t \in \sigma_{+} \text {or } s, t \in \sigma_{-}, \\ -1 /(s-t), & s \in \sigma_{+}, t \in \sigma_{-} \text {or } s \in \sigma_{-}, t \in \sigma_{+} .\end{cases}
$$

The operator $A_{0}$ certainly need not be bounded. However, the following lemma allows us to change the measure $\varrho$ so that $A_{0}$ becomes a Hilbert-Schmidt operator. We extend $A_{0}$ to $\mathcal{K}$ by putting $A_{0} \mid \mathcal{K}_{0}^{\frac{1}{2}}=\mathbf{0}$.

LEMMA 4.1. There exists $w \in L^{1}(\varrho), w>0, \varrho-a . e .$, such that the integral operator

$$
f \mapsto \int_{\sigma(W)} k(s, t) f(t) d \tilde{\varrho}(t)
$$

is a Hilbert-Schmidt operator on $L^{2}(\tilde{\varrho})$, where $d \tilde{\varrho}=w d \varrho$.

We postpone the proof of Lemma 4.1 until §II.6. 
Let us define the vectors $b$ and $c$ in $\mathcal{K}$ by

$$
c(t)=e_{1}, \quad b(t)=J(t) c(t)=\xi(t) c(t), \quad t \in \sigma(W)
$$

It is easy to see that

$$
A_{0}^{*}=J A_{0} \boldsymbol{J} \text { and } A_{0}^{*} W+W A_{0}=-C^{*} C .
$$

However, $\operatorname{Ker} A_{0}=\mathcal{K}_{0}^{\perp}$ is non-trivial except for the case when $W$ has simple spectrum. Therefore $A_{0}$ is not asymptotically stable in general. To overcome this obstacle we perturb $A_{0}$ by an operator $D$ such that the perturbed operator still satisfies (4.3) and becomes asymptotically stable. Of course, to make $A$ asymptotically stable, it is not sufficient to kill the kernel.

Let $\left\{a_{k}^{(n)}\right\}_{k=1}^{n-1}, n \in \mathbf{N} \cup \infty$, be positive numbers such that

$$
\sum_{k=1}^{n-1}\left(a_{k}^{(n)}\right)^{2}<\frac{1}{2 n^{2}}, \quad n \in \mathbf{N}, \quad \sum_{k=1}^{\infty}\left(a_{k}^{(\infty)}\right)^{2}<\infty .
$$

We define $D$ as multiplication by the operator-valued function $d$ :

$$
d(t)=\left(\begin{array}{ccccc}
0 & a_{1}^{(n)} & 0 & 0 & \ldots \\
-a_{1}^{(n)} & 0 & a_{2}^{(n)} & 0 & \ldots \\
0 & -a_{2}^{(n)} & 0 & a_{3}^{(n)} & \ldots \\
0 & 0 & -a_{3}^{(n)} & 0 & \ldots \\
\vdots & \vdots & \vdots & \vdots & \ddots
\end{array}\right)
$$

in the basis $\left\{e_{k}\right\}_{1 \leqslant k<n(t)+1}$, where $n=\nu_{W}(t)=\operatorname{dim} E(t)$.

Theorem 4.2. Let $A=A_{0}+D$, where $D$ is defined by (4.5) and suppose that (4.4) holds. Then

$$
A^{*} W+W A=-C^{*} C, \quad A^{*}=\boldsymbol{J} A \boldsymbol{J}
$$

and $A$ is asymptotically stable.

As we have already observed in §II.3, Theorem 4.2 implies the following result which solves the problem modulo the kernel.

CoRollaRY. Let $\Gamma_{h}$ be the Hankel operator associated with the linear system constructed above. Then $\Gamma_{h} \mid\left(\operatorname{Ker} \Gamma_{h}\right)$ is unitarily equivalent to $J W=\widetilde{\Gamma}$.

Proof of Theorem 4.2. Clearly $D$ commutes with $W$ and $D^{*}=-D$. So the operator $A=A_{0}+D$ satisfies the Lyapunov equation

$$
A^{*} W+W A=-C^{*} C
$$


It is easy to show that $D^{*}=\boldsymbol{J} D \boldsymbol{J}$ which implies $A^{*}=\boldsymbol{J} A \boldsymbol{J}$.

It remains to prove that $A$ is asymptotically stable. Let us show first that $A+A^{*} \leqslant 0$ which would imply that $\left\{e^{t A}\right\}_{t \geqslant 0}$ is a semigroup of contractions. We need the following lemma.

LEMMA 4.3. Let $W$ be a non-negative self-adjoint operator with trivial kernel and let $R$ be a self-adjoint operator such that

$$
R W+W R \leqslant 0
$$

Then $R \leqslant 0$.

Proof. Let $K=-(R W+W R)$. Since $-W \leqslant \mathbf{0}$ and $\operatorname{Ker} W=\{\mathbf{0}\}$, it follows that $-W$ is asymptotically stable. So we can apply Theorems 3.1 and 3.2 where $-W$ plays the role of $A$ and $-R$ plays the role of $W$. Then we find that $R$ is the unique solution of the equation

$$
X W+W X=-K
$$

and the solution is given by

$$
R=-\int_{\mathbf{R}} e^{-t W} K e^{-t W} d t
$$

Hence $R \leqslant \mathbf{0}$.

It follows from (4.6) and from the identity

$$
A W+W^{*} A=-B B^{*}
$$

that

$$
\left(A+A^{*}\right) W+W\left(A+A^{*}\right)=-C^{*} C-B B^{*} \leqslant 0
$$

and so $R=A+A^{*}$ satisfies the hypotheses of Lemma 4.3. Thus $A+A^{*} \leqslant 0$.

It is well-known that the above inequality implies that $\sigma(A) \subset\{\zeta: \operatorname{Re} \zeta \leqslant 0\}$.

Let us show that $A$ has no eigenvalues on the imaginary axis. Let

$$
A x=i \omega x, \quad x \in \mathcal{K}, x \neq \mathbf{0}, \omega \in \mathbf{R}
$$

Then

$$
\begin{aligned}
-\left(C^{*} C x, x\right) & =\left(A^{*} W x, x\right)+(W A x, x) \\
& =(W x, A x)+(A x, W x)=-i \omega(x, W x)+i \omega(x, W x)=0,
\end{aligned}
$$


and so $C x=0$, i.e. $x \perp c$. Similarly, if

$$
A^{*} \tilde{x}=i \tilde{\omega} \tilde{x}, \quad \tilde{x} \in \mathcal{K}, \tilde{\omega} \in \mathbf{R},
$$

then $B^{*} \tilde{x}=0$, i.e. $\tilde{x} \perp b$.

Applying equality (4.6) to the eigenvector $x$ and taking into account that $C x=0$, we obtain

$$
A^{*} W x=-i \omega W x
$$

i.e. $W x$ is an eigenvector of $A^{*}$. Now applying equality (4.7) to the eigenvector $W x$ and taking into account that $B^{*} W x=0$, we obtain

$$
A W^{2} x=i \omega W^{2} x .
$$

Repeating this procedure, we obtain

$$
A W^{2 n} x=i \omega W^{2 n} x, \quad n \geqslant 0 .
$$

It follows that for any eigenvector $x$ of $A$ with eigenvalue $i \omega$ and for any bounded measurable function $\varphi$

$$
A \varphi(W) x=i \omega \varphi(W) x .
$$

Consider the representation of $x$ in the direct integral:

$$
x(t)=\sum_{k=1}^{n(t)} x_{k}(t) e_{k} .
$$

It follows from (4.8) that $\varphi(W) x$ is orthogonal to $c$, i.e.

$$
\int \varphi(t) x_{1}(t) d \varrho(t)=0
$$

for any measurable $\varphi$. It follows that $x_{1}(t)=0$ a.e., i.e. $x \perp \mathcal{K}_{0}$. Hence $A x=D x$ and so $D x=i \omega x$ which means

$$
\left(\begin{array}{ccccc}
0 & a_{1}^{(n)} & 0 & 0 & \ldots \\
-a_{1}^{(n)} & 0 & a_{2}^{(n)} & 0 & \ldots \\
0 & -a_{2}^{(n)} & 0 & a_{3}^{(n)} & \ldots \\
0 & 0 & -a_{3}^{(n)} & 0 & \ldots \\
\vdots & \vdots & \vdots & \vdots & \ddots
\end{array}\right)\left(\begin{array}{c}
0 \\
x_{2}(t) \\
x_{3}(t) \\
x_{4}(t) \\
\vdots
\end{array}\right)=i \omega\left(\begin{array}{c}
0 \\
x_{2}(t) \\
x_{3}(t) \\
x_{4}(t) \\
\vdots
\end{array}\right)
$$

where $x(t)=\sum_{k=1}^{n} x_{k}(t) e_{k}, n=\nu_{W}(t)=\operatorname{dim} E(t)$. 
Performing the multiplication, we obtain

$$
\left(\begin{array}{c}
a_{1}^{(n)} x_{2}(t) \\
a_{2}^{(n)} x_{3}(t) \\
-a_{2}^{(n)} x_{2}(t)+a_{3}^{(n)} x_{4}(t) \\
-a_{3}^{(n)} x_{3}(t)+a_{4}^{(n)} x_{5}(t) \\
\vdots
\end{array}\right)=i \omega\left(\begin{array}{c}
0 \\
x_{2}(t) \\
x_{3}(t) \\
x_{4}(t) \\
\vdots
\end{array}\right)
$$

Comparing the components from top to bottom, we see that $x_{k}(t)=0$ for any $k$, i.e. $x=0$.

To prove that $A$ is asymptotically stable, we need the following result which follows easily from Proposition 6.7 in Chapter II of [SF] by applying the Cayley transform:

Stability test. Let $\left\{e^{t A}\right\}_{t \geqslant 0}$ be a strongly continuous semigroup of contractions on a Hilbert space $\mathcal{K}$ such that

(i) the spectrum $\sigma(A)$ of its generator is contained in $\{\zeta: \operatorname{Re} \zeta \leqslant 0\}$;

(ii) $A$ has no eigenvalues on the imaginary axis $i \mathbf{R}$;

(iii) the set $\sigma(A) \cap i \mathbf{R}$ is at most countable.

Then the semigroup $\left\{e^{t A}\right\}_{t \geqslant 0}$ is asymptotically stable, i.e.

$$
\lim _{t \rightarrow \infty}\left\|e^{t A} x\right\|=0 \quad \text { for any } x \in \mathcal{K} .
$$

We have already proved that our operator $A$ satisfies (i) and (ii). It remains to show that $A$ satisfies (iii) provided (4.3) holds.

Let $D_{n}$ be the operator on $\operatorname{span}\left\{e_{k}: 1 \leqslant k<n+1\right\}, 1 \leqslant n \leqslant \infty$, given by the matrix

$$
D_{n}=\left(\begin{array}{ccccc}
0 & a_{1}^{(n)} & 0 & 0 & \ldots \\
-a_{1}^{(n)} & 0 & a_{2}^{(n)} & 0 & \ldots \\
0 & -a_{2}^{(n)} & 0 & a_{3}^{(n)} & \ldots \\
0 & 0 & -a_{3}^{(n)} & 0 & \ldots \\
\vdots & \vdots & \vdots & \vdots & \ddots
\end{array}\right)
$$

We have $D^{*}=-D$ (i.e. $i D$ is self-adjoint) and so

$$
\sigma(D) \subset \operatorname{clos}\left(\bigcup_{n=1}^{\infty} \sigma\left(D_{n}\right)\right) \cup \sigma\left(D_{\infty}\right) .
$$

Clearly $D_{n}$ is a Hilbert-Schmidt operator for $1 \leqslant n \leqslant \infty$ and its Hilbert-Schmidt norm $\left\|D_{n}\right\|_{2}$ satisfies $\left\|D_{n}\right\|_{2} \leqslant 1 / n$ for $n<\infty$.

Therefore $\sigma\left(D_{n}\right) \subset[-i / n, i / n], n<\infty, \sigma\left(D_{n}\right)$ is finite for any $n<\infty, \sigma\left(D_{\infty}\right)$ is countable and can accumulate only at 0 . Hence the only possible accumulation point of the set $\left(\bigcup_{n=1}^{\infty} \sigma\left(D_{n}\right)\right) \cup \sigma\left(D_{\infty}\right)$ is 0 . Consequently, $\sigma(D)$ is at most countable. 
Since $A_{0}$ is a Hilbert-Schmidt operator, $A$ is a compact perturbation of $D$. So the essential spectrum $\sigma_{e}(A)$ of $A$ is equal to $\sigma_{e}(D)$ and for any $\lambda \in \sigma_{e}(A)$ we have ind $(A-\lambda I)=0$ (see [D2]). So if $\lambda \in \sigma(A) \backslash \sigma_{e}(A)$, then $\lambda$ must be an eigenvalue of $A$. We have already proved that $A$ has no eigenvalues on $i \mathbf{R}$ which implies that

$$
\sigma(A) \cap i \mathbf{R}=\sigma_{e}(A) \cap i \mathbf{R}=\sigma_{e}(D) \cap i \mathbf{R} \subset \sigma(D) \cap i \mathbf{R}
$$

which is at most countable.

\section{II.5. The kernel of $\boldsymbol{\Gamma}_{h}$}

In this section we shall prove Theorem 1.1. In the previous section we have constructed a linear system $\{A, B, C\}$ such that the corresponding Hankel operator $\Gamma_{h}$ restricted to $\left(\operatorname{Ker} \Gamma_{h}\right)^{\perp}$ is unitarily equivalent to $\Gamma \mid(\operatorname{Ker} \Gamma)^{\perp}$. To prove Theorem 1.1 completely we have to solve the problem of the description of $\operatorname{Ker} \Gamma_{h}$. The solution of this problem is given by the following theorem where we consider the Hankel operator $\Gamma_{h}$ associated with the system $\{A, B, C\}$ we have constructed in the previous section.

THEOREM 5.1. Let $\varrho$ be the scalar spectral measure of $W$ in (4.1). Then $\operatorname{Ker} \Gamma_{h}=$ $\{0\}$ if and only if $\int_{\sigma(W)}(1 / s) d \varrho(s)=\infty$.

Theorem 1.1 follows now from Theorem 5.1 and the following lemma.

Lemma 5.2. Let $\varrho$ be a finite positive Borel measure on $[0, a], a>0$, such that $0 \in$ supp $\varrho$ and

$$
\iint(k(s, t))^{2} d \varrho(s) d \varrho(t)<\infty
$$

where $k$ is defined by (4.2). Then we can change $\varrho$ by multiplying it by a positive weight in $L^{1}(\varrho)$ so that (5.1) still holds and.

$$
\int \frac{1}{s} d \varrho(s)=\infty .
$$

Remark. It is obvious that under the hypotheses of Lemma 5.2 one can change a measure $\varrho$ (by multiplying it by a positive weight in $L^{1}(\varrho)$ ) so that $(5.1)$ holds and

$$
\int \frac{1}{s} d \varrho(s)<\infty
$$

Indeed it is sufficient to take a weight which is sufficiently small near the origin.

The proof of Lemma 5.2 will be given in $§ I I .6$. Let us first derive Theorem 1.1 and then prove Theorem 5.1. 
Proof of Theorem 1.1. Let $\Gamma$ be a self-adjoint operator which satisfies the assumptions of Theorem 1.1. Put $\widetilde{\Gamma}=\Gamma \mid(\operatorname{Ker} \Gamma)^{\perp}$. Let $W=|\widetilde{\Gamma}|$ and consider the representation of $W$ in the form (4.1) where $\varrho$ is a scalar spectral measure of $W$. Let $A, B, C, J$ be as in $\S I I .4$ and let $\Gamma_{h}$ be the Hankel operator associated with the system $\{A, B, C\}$.

Suppose that $W$ is invertible. Since $\Gamma$ is non-invertible, the subspace $\operatorname{Ker} \Gamma$ is infinitedimensional. The operator $\Gamma_{h} \mid\left(\operatorname{Ker} \Gamma_{h}\right)^{\perp}$ being unitarily equivalent to $W J$ is also invertible. So $\operatorname{Ker} \Gamma_{h}$ is infinite-dimensional which implies that $\Gamma_{h}$ is unitarily equivalent to $\Gamma$.

Suppose now that $W$ is non-invertible. If $\operatorname{Ker} \Gamma=\{0\}$, we can choose by Lemma 5.2 a scalar spectral measure $\varrho$ of $W$ so that $\int(1 / s) d \varrho(s)=\infty$. Then by Theorem 5.1, $\operatorname{Ker} \Gamma_{h}=\{\mathbf{0}\}$ and so $\Gamma_{h}$ is unitarily equivalent to $\Gamma$.

If $\Gamma$ has an infinite-dimensional kernel, then by the remark to Lemma 5.2 we can choose $\varrho$ so that $\int(1 / s) d \varrho(s)<\infty$ and by Theorem $5.1, \Gamma_{h}$ has an infinite-dimensional kernel and again $\Gamma_{h}$ is unitarily equivalent to $\Gamma$.

Proof of Theorem 5.1. Let us first prove that $\operatorname{Ker} \Gamma_{h}$ is non-trivial provided $\int(1 / s) d \varrho(s)<\infty$. Assume that $\operatorname{Ker} \Gamma_{h}=\{0\}$. Since $\Gamma_{h}=V_{o} V_{c}^{*}=V_{o} J V_{o}^{*}$, it follows that $\operatorname{Ker} V_{o}^{*}=\{0\}$ which is equivalent to the fact that $V_{o}$ has dense range in $L^{2}\left(\mathbf{R}_{+}\right)$.

Let $\left\{\Phi_{t}\right\}_{t \geqslant 0}$ be the semigroup of backward translations on $L^{2}\left(\mathbf{R}_{+}\right),\left(\Phi_{t} f\right)(s)=$ $f(s+t), s, t \geqslant 0$. It is easy to see that

$$
V_{o} e^{t A}=\Phi_{t} V_{o}
$$

Clearly the condition $\int(1 / s) d \varrho(s)<\infty$ means that $c \in$ Range $W^{1 / 2}$.

Let $V_{o}=U_{o} W^{1 / 2}$ be the polar decomposition at $V_{o}$ (see the proof of Theorem 2.1). Since $\operatorname{Ker} \Gamma_{h}=\{0\}$, it follows that $U_{o}$ is unitary. Therefore $c \in \operatorname{Range} V_{o}^{*}$. Let $c=V_{o}^{*} f$, $f \in L^{2}(\mathbf{R})$. Define the operator $F: L^{2}(\mathbf{R}) \rightarrow \mathbf{C}$ by

$$
F \varphi=(\varphi, f)
$$

Then obviously $F V_{o}=C$, where as above $C x=(x, c)$. Therefore by $(5.2)$

$$
C e^{t A}=F \Phi_{t} V_{o}
$$

and so

$$
e^{t A^{*}} C^{*} C e^{t A}=V_{o}^{*} \Phi_{t}^{*} F^{*} F \Phi_{t} V_{o}
$$

which implies

$$
\int_{\mathbf{R}_{+}} e^{t A^{*}} C^{*} C e^{t A} d t=W=V_{o}^{*}\left(\int_{\mathbf{R}_{+}} \Phi_{t}^{*} F^{*} F \Phi_{t} d t\right) V_{o}
$$


Since $V_{o}=U_{o} W^{1 / 2}$ and $V_{o}^{*}=W^{1 / 2} U_{o}^{*}$, we have

$$
U_{o}^{*}\left(\int_{\mathbf{R}} \Phi_{t}^{*} F^{*} F \Phi_{t} d t\right) U_{o}=I
$$

and bearing in mind that $U_{o}$ is unitary, we obtain

$$
\int_{\mathbf{R}_{+}} \Phi_{t}^{*} F^{*} F \Phi_{t} d t=I
$$

Consider the function $f_{\tau}, \tau \geqslant 0$, defined by

$$
f_{\tau}(t)= \begin{cases}f(t-\tau), & t \geqslant \tau, \\ 0, & t<\tau,\end{cases}
$$

and the operators $F_{\tau}: L^{2}(\mathbf{R}) \rightarrow \mathbf{C}$ defined by

$$
F_{\tau} x=\left(x, f_{\tau}\right) .
$$

Since $F \Phi_{\tau}=F_{\tau}$, identity (5.3) can be rewritten as

$$
\int_{\mathbf{R}_{+}} F_{\tau}^{*} F_{\tau} d \tau=I
$$

The operator $F_{\tau}^{*} F_{\tau}$ is the integral operator with kernel $\varkappa_{\tau}(s, t)=f_{\tau}(s) \overline{f_{\tau}(t)}$. We have

$$
\varkappa(s, t) \stackrel{\text { def }}{=} \int_{0}^{\infty} \varkappa_{\tau}(s, t) d \tau=\int_{0}^{\infty} f_{\tau}(s) \overline{f_{\tau}(t)} d \tau=\int_{0}^{\min \{s, t\}} f(s-\tau) \overline{f(t-\tau)} d \tau,
$$

hence

$$
|\varkappa(s, t)| \leqslant\|f\|_{2}^{2}
$$

Therefore (5.4) implies that

$$
(\varphi, \psi)=\int_{0}^{\infty} \int_{0}^{\infty} \varkappa(s, t) \varphi(s) \overline{\psi(t)} d s d t
$$

at least for compactly supported $\varphi$ and $\psi$ in $L^{2}$.

Let now

$$
\varphi(s)=\psi(s)= \begin{cases}1 / \sqrt{\varepsilon}, & 0 \leqslant s \leqslant \varepsilon \\ 0, & s>\varepsilon\end{cases}
$$

Then (5.5) implies that

$$
1=\|\varphi\|_{2}^{2}=(\varphi, \varphi) \leqslant \varepsilon\|\varkappa\|_{\infty} \leqslant \varepsilon\|f\|_{2}^{2}<1
$$

for a sufficiently small $\varepsilon$. The contradiction obtained proves that $\operatorname{Ker} \Gamma_{h} \neq\{0\}$.

Let us show that if $\operatorname{Ker} \Gamma_{h} \neq\{0\}$, then $\int(1 / s) d \varrho(s)<\infty$. It is easy to see that the subspace $K=\left(\operatorname{Ker} \Gamma_{h}\right)^{\perp}$ is invariant under the semigroup of backward translations $\left\{\Phi_{s}\right\}_{s} \geqslant 0$. Let $\Psi_{s} \stackrel{\text { def }}{=} \Phi_{s} \mid \boldsymbol{K}$. Since Ker $\Gamma_{h}=\operatorname{Ker} V_{o}^{*}$, it follows that $K=$ clos Range $V_{0}^{*}$ and so by (5.2)

$$
V_{o} e^{t A}=\Psi_{t} V_{o}
$$

We need the following lemma. 
LEMMA 5.3. The semigroup $\left\{\Psi_{t}\right\}_{t \geqslant 0}$ has a bounded generator.

Let us first complete the proof of Theorem 5.1 and then prove Lemma 5.3. By Lemma 5.3, $\Psi_{t}=e^{t G}, t \geqslant 0$. It follows from (5.6) that

$$
V_{o} A=G V_{o}
$$

and so

$$
G=V_{o} A V_{o}^{-1}
$$

( $V_{o} A V_{o}^{-1}$ is defined on a dense subset of $\boldsymbol{K}$ and extends by continuity to a bounded operator).

Put

$$
R \stackrel{\text { def }}{=} G+G^{*}=V_{o} A V_{o}^{-1}+\left(V_{o}^{-1}\right)^{*} A^{*} V_{o}^{*} .
$$

Multiplying this equality by $V_{o}$ on the right and $V_{o}^{*}$ on the left and bearing in mind that $V_{o}^{*} V_{o}=W$, we obtain

$$
W A+A^{*} W=V_{o}^{*} R V_{o}
$$

On the other hand, we have from (4.6)

$$
W A+A^{*} W=-C^{*} C=-(\cdot, c) c .
$$

So $V_{o}^{*} R V_{o}=-C^{*} C$. Hence $c \in \operatorname{Range} V_{o}^{*}$ or, which is equivalent, $c \in \operatorname{Range} W^{1 / 2}$ (see the proof of Theorem 2.1). Clearly the last condition exactly means that

$$
\int \frac{1}{s} d \varrho(s)<\infty
$$

Proof of Lemma 5.3. To show that the generator of our contractive semigroup is bounded, we apply inverse Fourier transformation $\mathcal{F}^{-1}$ which maps $L^{2}\left(\mathbf{R}_{+}\right)$onto the Hardy class $H^{2}=H^{2}\left(\mathbf{C}_{+}\right)$. Put $\breve{\Phi}_{s}=\mathcal{F}^{-1} \Phi_{s} \mathcal{F}$ and $\breve{\Psi}_{s}=\mathcal{F}^{-1} \Psi_{s} \mathcal{F}$. Then

$$
\check{\Psi}_{s} f=\mathbf{P}_{+} e_{-s} f,
$$

where $e_{-s}(t)=e^{-i s t}$ and $\mathbf{P}_{+}$is the orthogonal projection from $L^{2}$ onto $H^{2}$. Clearly

$$
\check{\Psi}_{s}=\check{\Phi}_{s} \mid \check{K}
$$

where $\check{\boldsymbol{K}}=\mathcal{F}^{-1} \boldsymbol{K}$.

Obviously $\check{\boldsymbol{K}}$ is an invariant subspace of the semigroup $\left\{\check{\Phi}_{s}\right\}_{s \geqslant 0}$ and so by Lax's theorem (see e.g. [N, Chapter XI]) $\check{K}$ has the form $\boldsymbol{K}_{\vartheta}=H^{2} \ominus \vartheta H^{2}$, where $\vartheta$ is an inner function. 
Let $\check{G}$ be the generator of the semigroup $\left\{\check{\Psi}_{s}\right\}_{s \geqslant 0}$. It is sufficient to show that $\check{G}$ is bounded. To this end we consider its Cayley transform $T=(\breve{G}+I)(\breve{G}-I)^{-1}$ which is called the cogenerator of the semigroup $\left\{\breve{\Psi}_{s}\right\}_{s \geqslant 0}$. Since $\breve{G}=(T+I)(T-I)^{-1}$, it is sufficient to show that $1 \notin \sigma(T)$.

The cogenerator $T$ can be found easily. According to a theorem by Sz.-Nagy and Foias, see [SF, Chapter III, Theorem 8.1], the cogenerator $T$ of a semigroup of contractions $\{T(s)\}_{s \geqslant 0}$ is given by

$$
T=\lim _{s \rightarrow 0+} \varphi_{s}(T(s)),
$$

where

$$
\varphi_{s}(\lambda)=\frac{\lambda-1+s}{\lambda-1-s}
$$

Taking into account that

$$
\varphi\left(\check{\Psi}_{s}\right) f=\mathbf{P}_{+} \varphi\left(e_{-s}\right) f, \quad f \in \boldsymbol{K}_{\vartheta}
$$

we can conclude that the cogenerator $T$ of the semigroup $\check{\Psi}_{s}$ is given by the formula

$$
T f=\mathbf{P}_{+} \varphi f, \quad f \in \boldsymbol{K}_{\vartheta}
$$

where $\varphi(t)=\lim _{s \rightarrow 0+} \varphi_{s}\left(e^{-i s t}\right)=(s+i) /(s-i)$.

Let $\omega$ be the conformal map of the unit disc $\mathbf{D}$ onto the half-plane $\mathbf{C}_{+}$defined by

$$
\omega(z)=i \frac{1+z}{1-z} .
$$

Let $\tilde{\vartheta} \stackrel{\text { def }}{=} \vartheta \circ \omega$. Note that $\tilde{\vartheta}$ is an inner function in $H^{\infty}(\mathbf{D})$. Then the unitary operator $\mathcal{U}$,

$$
(\mathcal{U} f)(t)=\pi^{1 / 2} \frac{1}{t+i} f \circ \omega^{-1}=\pi^{1 / 2} \frac{1}{t+i} f\left(\frac{t-i}{t+i}\right), \quad t \in \mathbf{R}
$$

maps unitarily $H^{2}(\mathbf{D})$ onto $H^{2}\left(\mathbf{C}_{+}\right)$and $\boldsymbol{K}_{\tilde{\vartheta}}$ onto $\boldsymbol{K}_{\vartheta}$, and moreover $\mathcal{U}^{-1} T \mathcal{U} f=S^{*} \mid \boldsymbol{K}_{\tilde{\vartheta}}$, where $S^{*}$ is backward shift,

$$
S^{*} f=\mathbf{P}_{+} \bar{z} f(z)=\frac{f-f(0)}{z}, \quad f \in H^{2} .
$$

So, $T$ is unitarily equivalent to $S^{*} \mid \boldsymbol{K}_{\tilde{\vartheta}}$.

To complete the proof we need the notion of the spectrum of an inner function. Let $\tau$ be an inner function. Then $\tau=\alpha B s$, where $\alpha \in \mathbf{C},|\alpha|=1, B$ is a Blaschke product with zero set $\Lambda$ and $s$ is a singular function, i.e.

$$
s(z)=\exp \left(-\int_{\mathbf{T}} \frac{\zeta+z}{\zeta-z} d \lambda(\zeta)\right)
$$


for some positive measure $\lambda$ which is singular with respect to Lebesgue measure. The spectrum $\sigma(\tau)$ of $\tau$ is by definition clos $\Lambda \cup \operatorname{supp} \lambda$.

It is well known (see [N, Chapter III]) that the spectrum of $S^{*} \mid\left(H^{2} \ominus \tau H^{2}\right)$ is equal to $\overline{\sigma(\tau)}$. Therefore in our case $\sigma(T)=\overline{\sigma(\tilde{\vartheta})}$.

If $\vartheta$ is an inner function in $\mathbf{C}_{+}$, we put by definition $\sigma(\vartheta)=\omega(\sigma(\tilde{\vartheta}))$, the spectrum of $\vartheta$. Note that $\sigma(\vartheta)$ can contain $\infty$ (this happens if and only if $1 \in \sigma(\tilde{\vartheta})$ ).

Let us prove that $\sigma(\vartheta) \subset-i \overline{\sigma(A)}$ which would imply that $\sigma(\vartheta)$ is bounded and since

$$
\sigma(T)=\overline{\sigma(\tilde{\vartheta})}=\omega^{-1}(\sigma(\vartheta))
$$

it would follow that $1 \notin \sigma(T)$ and so $G$ is bounded.

To this end we need the notion of pseudocontinuation. Recall (see [N, Lecture II]) that a function $f \in H^{p}\left(\mathbf{C}_{+}\right), 1 \leqslant p \leqslant \infty$, is said to have a meromorphic psedocontinuation (or simply, pseudocontinuation) if there exists a meromorphic function $g$ of Nevanlinna class $\mathcal{N}\left(\mathbf{C}_{-}\right)$in $\mathbf{C}_{-}\left(\mathcal{N}\left(\mathbf{C}_{-}\right) \stackrel{\text { def }}{=}\left\{g=g_{1} / g_{2}: g_{1}, g_{2} \in H^{\infty}\left(\mathbf{C}_{-}\right)\right\}\right)$such that the boundary values of $g$ coincide with the boundary values of $f$ almost everywhere on $\mathbf{R}$. Such a function $g$ is called a pseudocontinuation of $f$.

Each inner function $\vartheta$ a can be extended to $\mathbf{C}_{-}$by

$$
\vartheta(\zeta)=1 / \overline{\vartheta(\bar{\zeta})}, \quad \zeta \in \mathbf{C}_{-}
$$

Such an extension is a meromorphic function with poles at the points conjugate to the zeroes of $\vartheta$ in $\mathbf{C}_{+}$, and clearly it belongs to $\mathcal{N}\left(\mathbf{C}_{-}\right)$. It is also easy to see that this extension has non-tangential boundary values almost everywhere and those boundary values coincide a.e. with $\vartheta$ on $\mathbf{R}$, that is, each inner function has a pseudocontinuation.

A function $f \in H^{2}\left(\mathbf{C}_{+}\right)$belongs to $\boldsymbol{K}_{\vartheta}$ if and only if $f / \vartheta \in H^{2}\left(\mathbf{C}_{-}\right)$. Since $H^{2}\left(\mathbf{C}_{-}\right) \subset$ $\mathcal{N}\left(\mathbf{C}_{-}\right)$, it is easy to see that if $f \in \boldsymbol{K}_{\vartheta}$, then $f=(f / \vartheta) \vartheta$ has a pseudocontinuation.

We need the following well known facts:

(i) if a function $f \in H^{2}\left(\mathbf{C}_{+}\right)$has a pseudocontinuation and it extends analytically across an interval $I \subset \mathbf{R}$, then its extension coincides with its meromorphic pseudocontinuation;

(ii) if $\vartheta$ is an inner function such that $\mathbf{R} \not \subset \sigma(\vartheta)$, then $\vartheta$ extends analytically to $\mathbf{C} \backslash \overline{\sigma(\vartheta)}$;

(iii) if $\mathbf{R} \not \subset \sigma(\vartheta)$ and $f \in \boldsymbol{K}_{\vartheta}$, then $f$ extends analytically to $\mathbf{C} \backslash \overline{\sigma(\vartheta)}$.

The proofs of (i)-(iii) for $H^{p}$ functions on the unit disc can be found in [N, Lectures II, III]. It is easy to see that the case of functions in $\mathbf{C}_{+}$reduces easily to the case of the unit disc.

Now we are in a position to prove that $\sigma(\vartheta) \subset-i \overline{\sigma(A)}$. To do that we need the following lemma. Let $\widehat{\mathbf{C}}$ denote the Riemann sphere $\widehat{\mathbf{C}}=\mathbf{C} \cup\{\infty\}$. Till the end of the section we assume that a function $f \in \boldsymbol{K}_{\vartheta}$ is extended to $\mathbf{C}_{-}$as a meromorphic function. 
LemmA 5.4. Let $\vartheta$ be an inner function in $\mathbf{C}_{+}$and let $\sigma$ be a closed subset of its spectrum $\sigma(\vartheta), \sigma \neq \sigma(\vartheta)$. Then there exists a non-trivial inner divisor $\vartheta_{1}$ of $\vartheta$ (i.e. $\vartheta / \vartheta_{1} \neq$ const) such that every function $f \in \boldsymbol{K}_{\vartheta}$, which extends analytically to $\widehat{\mathbf{C}} \backslash \bar{\sigma}$, belongs to $\boldsymbol{K}_{\mathfrak{\vartheta}_{1}}$.

Let us first complete the proof of Lemma 5.3. Assume that $\sigma(\vartheta) \not \subset-i \overline{\sigma(A)}$. Put $\sigma=\sigma(\vartheta) \cap-i \overline{\sigma(A)}$. Let $\vartheta_{1}$ be an inner function whose existence follows from Lemma 5.4. Consider an arbitrary $f=\mathcal{F}^{-1} V_{o} x, x \in \mathcal{K}$. Since

$$
\left(\mathcal{F}^{-1} V_{o} x\right)(s)=\frac{1}{\sqrt{2 \pi}} \int_{\mathbf{R}_{+}} C e^{t A} x e^{i s t} d t=\frac{1}{\sqrt{2 \pi}}\left((A+i s I)^{-1} x, c\right), \quad \operatorname{Im} s>0,
$$

the function $f$ extends analytically outside the set $i \sigma(A)$. On the other hand, $f \in \boldsymbol{K}_{\vartheta}$ and and by (iii) it extends analytically to $\mathbf{C} \backslash \overline{\sigma(\vartheta)}$. Therefore $f$ extends analytically to $\widehat{\mathbf{C}} \backslash \vec{\sigma}$. By Lemma 5.4, $f \in \boldsymbol{K}_{\vartheta_{1}}$ and since $\left\{\mathcal{F}^{-1} V_{o} x: x \in \mathcal{K}\right\}$ is dense in $\boldsymbol{K}_{\vartheta}$, it follows that $\boldsymbol{K}_{\vartheta} \subset \boldsymbol{K}_{\vartheta_{1}}$ which contradicts the fact that $\vartheta_{1}$ is a non-trivial inner divisor of $\vartheta$. So $\sigma(\vartheta) \subset-i \overline{\sigma(A)}$ and as we have already noted, this implies the boundedness of $G$.

Remark. It can be shown that $\sigma(T)=\overline{\sigma(\tilde{\vartheta})}=\omega^{-1}(-i \overline{\sigma(A)})$ and $\sigma(T)$ must be symmetric about the real line: $\sigma(T)=\overline{\sigma(T)}$. Thus $\sigma(A)$ must be symmetric too: $\sigma(A)=\sigma\left(A^{*}\right)$.

Proof of Lemma 5.4. Note that $g \in \boldsymbol{K}_{\vartheta}$ if and only if $g \in H^{2}\left(\mathbf{C}_{+}\right)$and $g / \vartheta \in H^{2}\left(\mathbf{C}_{-}\right)$.

Consider first the simplest case. Suppose that there exists a point $\lambda$ in $\mathbf{C}_{+}$such that $\lambda \in \sigma(\vartheta) \backslash \sigma$. Then we can define $\vartheta_{1}$ as $\vartheta_{1}=\vartheta / b_{\lambda}$, where $b_{\lambda}(z)=(z-\lambda) /(z-\bar{\lambda})$ is a Blaschke factor.

Suppose that $f \in H^{2}\left(\mathbf{C}_{+}\right), f / \vartheta \in H_{-}^{2}$ and $f$ extends analytically to $\bar{\lambda}$. Since the pseudocontinuation of $\vartheta$ has a pole at $\bar{\lambda}$, it follows that $(f / \vartheta)(\bar{\lambda})=0$. The function $1 / b_{\lambda}$ in $\mathbf{C}_{-}$can be considered as a Blaschke factor with zero at $\bar{\lambda}$. Since the function $f / \vartheta$ belongs to $H^{2}\left(\mathbf{C}_{-}\right)$and vanishes at $\bar{\lambda}$, it follows that $1 / b_{\lambda}$ is a divisor of $f / \vartheta$ and so $f / \vartheta_{1}=b_{\lambda} f / \vartheta \in H^{2}\left(\mathbf{C}_{-}\right)$, which means that $f \in \boldsymbol{K}_{\vartheta_{1}}$.

Suppose now that $\mathbf{C}_{+} \cap(\sigma(\vartheta) \backslash \sigma)=\varnothing$. Put $\widehat{\mathbf{R}} \stackrel{\text { def }}{=} \mathbf{R} \cup\{\infty\}$. Then there exists an open connected set $I$ in $\widehat{\mathbf{R}}$ such that $I \cap \sigma(\vartheta) \neq \varnothing$ and $I$ is separated from $\sigma$.

Let $\tau$ be a nontrivial inner divisor of $\vartheta$ whose spectrum is contained in $I$ and let $\vartheta_{1}=\vartheta / \tau$. Let us prove that $\vartheta_{1}$ satisfies the conclusion of the lemma.

Let $f$ be a function in $\boldsymbol{K}_{\vartheta}$ which extends analytically to $\mathbf{C} \backslash \sigma$ and so $f$ is holomorphic in a neighbourhood of clos $I$. Since $f \in \mathcal{N}\left(\boldsymbol{C}_{-}\right), f$ can be represented as $f=g_{1} / g_{2}$, where $g_{1}, g_{2} \in H^{\infty}\left(\mathbf{C}_{-}\right)$and $g_{1}$ and $g_{2}$ have no nontrivial common inner factor. We need the following fact.

LEMMA 5.5. Let $\varphi_{1}$ and $\varphi_{2}$ be bounded analytic functions in $\mathbf{D}$ which have no common inner factor. If $\varphi_{1} / \varphi_{2}$ extends analytically to a neighbourhood of a closed arc $J$ in $\mathbf{T}$, then the spectra of the inner components of $\varphi_{1}$ and $\varphi_{2}$ are separated from $J$. 
Let us first complete the proof of Lemma 5.4. Applying the conformal map from C_ onto $\mathbf{D}$, we find from Lemma 5.5 that the spectra of the inner components of $g_{1}$ and $g_{2}$ are separated from $I$.

Let $\vartheta^{\#}$ and $\tau^{\#}$ be the inner functions in $\mathbf{C}_{-}$defined by $\vartheta^{\#}(z)=\overline{\vartheta(\bar{\zeta})}, \tau^{\#}(z)=\overline{\tau(\bar{\zeta})}$, $\zeta \in \mathbf{C}_{-}$. We have $f / \vartheta \in H^{2}\left(\mathbf{C}_{-}\right)$and $(f / \vartheta)(\zeta)=f(z) \vartheta^{\#}(\zeta), \zeta \in \mathbf{C}_{-}$. Since the spectrum of the inner factor of $g_{2}$ is separated from $I$, it is easy to see that $\tau^{\#}$ is a divisor of $g_{1}$. It follows that the function $f \vartheta^{\#} / \tau^{\#}$ in $\mathbf{C}_{-}$belongs to $H^{2}$. Clearly the boundary values of this function coincide with $\varphi / \vartheta_{1}$ which completes the proof.

It remains to prove Lemma 5.5 which is familiar to experts though we were unable to find a reference. The following simple proof is due to D. Marshall.

Proof of Lemma 5.5. By dividing $\varphi_{1}$ by a polynomial we can assume that $\varphi_{1}$ has no zeroes in a neighbourhood of $J$. Clearly the zeroes of $\varphi_{1}$ and $\varphi_{2}$ are separated from $J$. So we can divide $\varphi_{1}$ and $\varphi_{2}$ by the corresponding Blaschke products and reduce the situation to the case when $\varphi_{1}$ and $\varphi_{2}$ have no zeroes in $\mathbf{D}$. Then $\log \left|\varphi_{1} / \varphi_{2}\right|$ is the Poisson integral of a real measure $\nu$ on T. Clearly $\nu$ is the weak limit of $\nu_{r}$ as $r \rightarrow 1$, where $d \nu_{r}(\zeta)=\log \left|\varphi_{1}(r \zeta) / \varphi_{2}(r \zeta)\right| d m(\zeta)$. Since $\log \left|\varphi_{1} / \varphi_{2}\right|$ is smooth in a neighbourhood of $J$, it follows that the restrictions of $\nu_{r}$ to $J$ converge in the norm to the restriction of $\nu$ to $J$ and so $\nu$ is absolutely continuous on $J$ which means that the singular measures of the inner components of $\varphi_{1}$ and $\varphi_{2}$ are supported outside $J$.

\section{II.6. Exercises in measure theory}

The aim of this section is to prove Lemmas 4.1 and 5.2. They will be proved if we establish the following facts:

Let $\varrho$ be a finite positive Borel measure on $[0, a], a>0$, which has no mass at 0 and let $\sigma_{+}$and $\sigma_{-}$be disjoint sets such that $\sigma_{+} \cup \sigma_{-}=\operatorname{supp} \varrho$. Then there exists a weight $w \in L^{1}(\varrho)$ which is positive $\varrho$-a.e. and such that the measure $\tilde{\varrho}, d \tilde{\varrho}=w d \varrho$, satisfies:

(a) the integral operator $A_{0}$ on $L^{2}(\tilde{\varrho})$ with kernel (4.2) is Hilbert-Schmidt;

(b) if $0 \in \operatorname{supp} \varrho$, then we can find a $w$ so that, in addition to (a), $\int(1 / s) d \tilde{\varrho}(s)=\infty$.

Proof. We begin with (a). In the case when one of the sets $\sigma_{+}, \sigma_{-}$is empty the result is trivial. Assume that both $\sigma_{+}$and $\sigma_{-}$have positive measure. Let $\sigma_{+}^{(n)}$ and $\sigma_{-}^{(n)}$ be compact subsets of $\sigma_{+}$and $\sigma_{-}$such that $\sigma_{+}^{(n)} \subset \sigma_{+}^{(n+1)}, \sigma_{-}^{(n)} \subset \sigma_{-}^{(n+1)}, n \geqslant n+1$, and

$$
\lim _{n \rightarrow \infty} \varrho\left(\sigma_{+} \backslash \sigma_{+}^{(n)}\right)=\lim _{n \rightarrow \infty} \varrho\left(\sigma_{-} \backslash \sigma_{-}^{(n)}\right)=0 .
$$

Clearly the restriction of $k$ to $\left(\sigma_{+}^{(n)} \cup \sigma_{-}^{(n)}\right) \times\left(\sigma_{+}^{(n)} \cup \sigma_{-}^{(n)}\right)$ is bounded.

19-950233 Acta Mathematica 174. Imprimé le 20 juin 1995 
Suppose we have already defined $w$ on $\sigma_{+}^{(n)} \cup \sigma_{-}^{(n)}$. Let us define it on $\sigma_{+}^{(n+1)} \cup \sigma_{-}^{(n+1)}$. We can easily do that so that

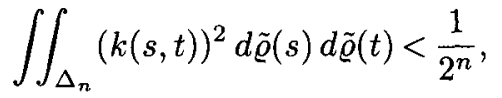

where $\Delta_{n}=\left(\sigma_{+}^{(n+1)} \cup \sigma_{-}^{(n+1)}\right) \times\left(\sigma_{+}^{(n+1)} \cup \sigma_{-}^{(n+1)}\right) \backslash\left(\sigma_{+}^{(n)} \cup \sigma_{-}^{(n)}\right) \times\left(\sigma_{+}^{(n)} \cup \sigma_{-}^{(n)}\right), d \tilde{\varrho}=w d \varrho$ and $w$ is positive a.e. on $\Delta_{n}$. Doing in this way, define $w$ on supp $\varrho$. Clearly the measure $\tilde{\varrho}$, $d \tilde{\varrho}=w d \mu$, satisfies (a). The above reasoning was suggested by Svante Janson.

To prove (b) we consider first the simplest case when one of the sets $\sigma_{+}, \sigma_{-}$is empty and so $k(s, t)=-1 /(s+t)$. Without loss of generality we can assume that supp $\varrho \subset[0,1]$.

Let $\delta_{n}=\left(2^{-n}, 2^{-n+1}\right], n \geqslant 1$. Consider the increasing sequence $\left\{n_{j}\right\}_{j \geqslant 1}$ of integers such that $\varrho\left(\delta_{n_{j}}\right)>0$ and $\varrho\left(\delta_{n}\right)=0$ if $n \neq n_{j}$ for any $j$. Since $0 \in \operatorname{supp} \varrho$, the sequence $\left\{n_{j}\right\}_{j \geqslant 1}$ is infinite. We define the weight $w$ by

$$
w(s)=\frac{2^{-n_{j}}}{\varrho\left(\delta_{n_{j}}\right) j \log (j+1)}, \quad s \in \delta_{n_{j}} .
$$

Then

$$
\begin{aligned}
\int_{0}^{1} \frac{w(s) d \varrho(s)}{s} & =\sum_{j \geqslant 1} \int_{\delta_{n_{j}}} \frac{w(s) d \varrho(s)}{s} \\
& =\sum_{j \geqslant 1} \frac{2^{-n_{j}}}{\varrho\left(\delta_{n_{j}}\right) j \log (j+1)} \int_{\delta_{n_{j}}} \frac{d \varrho(s)}{s} \\
& \geqslant \sum_{j \geqslant 1} \frac{2^{-n_{j}}}{\varrho\left(\delta_{n_{j}}\right) j \log (j+1)} \varrho\left(\delta_{n_{j}}\right) \cdot 2^{n_{j}-1}=\frac{1}{2} \sum_{j \geqslant 1} \frac{1}{j \log (j+1)}=\infty
\end{aligned}
$$

So

$$
\int_{0}^{1} \frac{d \tilde{\varrho}(s)}{s}=\infty
$$

On the other hand,

$$
\begin{aligned}
\iint & (k(s, t))^{2} d \varrho(s) d \varrho(t)=\sum_{j, r \geqslant 1} \int_{\delta_{n_{j}}} \int_{\delta_{n_{r}}} \frac{w(s) w(t)}{(s+t)^{2}} d \varrho(s) d \varrho(t) \\
\leqslant & 2 \sum_{1 \leqslant j<\infty, r \leqslant j} \frac{1}{\varrho\left(\delta_{n_{j}}\right) \varrho\left(\delta_{n_{r}}\right) j \log (j+1) r \log (r+1)} \\
& \times \int_{\delta_{n_{j}}} \int_{\delta_{n_{r}}} \frac{2^{-n_{j}} 2^{-n_{r}}}{\left(2^{\left.-n_{j}+2^{-n_{r}}\right)^{2}} d \varrho(s) d \varrho(t)\right.} \\
\leqslant & \sum_{1 \leqslant j<\infty, r \leqslant j} \frac{1}{\varrho\left(\delta_{n_{j}}\right) \varrho\left(\delta_{n_{r}}\right) j \log (j+1) r \log (r+1)} \cdot 2^{n_{r}} \cdot 2^{-n_{j}} \varrho\left(\delta_{n_{j}}\right) \varrho\left(\delta_{n_{r}}\right) \\
=2 & \sum_{1 \leqslant j<\infty} \sum_{1 \leqslant r \leqslant j} \frac{2^{n_{r}} 2^{-n_{j}}}{j \log (j+1) r \log (r+1)} .
\end{aligned}
$$


Since

$$
\sum_{r=1}^{j} \frac{2^{n_{r}}}{r \log (r+1)} \leqslant \frac{2^{n_{j}}}{\log (j+1)}
$$

we have

$$
\iint(k(s, t))^{2} d \tilde{\varrho}(s) d \underline{\varrho}(t) \leqslant 2 \sum_{j=1}^{\infty} \frac{1}{j \log ^{2}(j+1)}<\infty .
$$

Consider now the general case. Since $0 \in \operatorname{supp} \varrho, 0$ is an accumulation point of either $\sigma_{+}$or $\sigma_{-}$. To be definite, assume that 0 is an accumulation point of $\sigma_{+}$. Then we can consider the sets $\delta_{n}=\left(2^{-n}, 2^{-n+1}\right\} \cap \sigma_{+}$, the increasing sequence $\left\{n_{j}\right\}_{j \geqslant 1}$ such that $\varrho\left(\delta_{n_{j}}\right)>0$ and $\varrho\left(\delta_{n}\right)=0$ if $n \neq n_{j}$ for any $j$. We can define $w$ on $\delta_{n_{j}}$ as in (6.1). It follows from the above reasonings that

$$
\int_{\sigma_{+}} \frac{w(s) d \varrho(s)}{s}=\infty
$$

and

$$
\int_{\sigma_{+}} \int_{\sigma_{+}}(k(s, t))^{2} d \tilde{\varrho}(s) d \tilde{\varrho}(t)<\infty .
$$

We can now define $w$ inductively on $\left(2^{-n_{j}}, 2^{-n_{j-1}}\right] \cap \sigma_{-}$to be so small that

$$
\begin{aligned}
\int_{2^{-n_{j}}}^{1} \int_{2^{-n_{j}}}^{1}(k(s, t))^{2} w(s) w(t) d \varrho(s) & d \varrho(t) \\
& \leqslant 2\left(1-2^{-j}\right) \int_{\Delta_{j}} \int_{\Delta_{j}}(k(s, t))^{2} w(s) w(t) d \varrho(s) d \varrho(t),
\end{aligned}
$$

where $\Delta_{j}=\sigma_{+} \cap\left(2^{-n_{j}}, 1\right]$. Obviously this can easily be done.

\section{II.7. Positive Hankel operators with multiple spectrum}

Here we prove that no positive Hankel operator with multiple spectrum (i.e. the set $\{t>0: \nu(t)>1\}$ has positive measure) can be realized by a balanced realization $\{A, B, C\}$ with one-dimensional input and one-dimensional output. Recall that we consider only systems with bounded $A, B$, and $C$.

THEOREM 7.1. Let $\Gamma$ be a non-negative self-adjoint operator such that $W=$ $\Gamma \mid(\operatorname{Ker} \Gamma)^{\perp}$ has multiple spectrum. Then there exists no balanced system $\{A, B, C\}$ with one-dimensional input and one-dimensional output such that $\Gamma_{h} \mid\left(\operatorname{Ker} \Gamma_{h}\right)^{\perp}$ is unitarily equivalent to $W$.

Recall that $\Gamma_{h}$ is the Hankel operator associated with $\{A, B, C\}$. 
Theorem 7.1 shows that not all self-adjoint Hankel operators (even up to unitary equivalence) can be realized by a balanced system with continuous time.

Proof. Suppose that $W$ is unitarily equivalent to $\Gamma_{h} \mid\left(\operatorname{Ker} \Gamma_{h}\right)^{\perp}$ for some balanced system $\{A, B, C\}$. Then by Theorems 2.2 and $2.4, J=I, A=A^{*}, b=c$. We have

$$
W=\int_{0}^{\infty} e^{t A} C^{*} C e^{t A} d t
$$

the integral being convergent in the weak operator topology.

Let us show that $\operatorname{Ker} A=\{0\}$. Indeed if $x \in \operatorname{Ker} A$, then

$$
\int_{0}^{\infty}\left(e^{t A} C^{*} C e^{t A} x, x\right) d t=\int_{0}^{\infty}\left|\left(e^{t A} x, c\right)\right|^{2} d t=\int_{0}^{\infty}|(x, c)|^{2} d t<\infty
$$

so $x \perp e^{t A} c$ and since $c$ is a cyclic vector of $A$, we have $x=0$.

As we have already proved,

$$
A W+W A=-C^{*} C
$$

(see §II.3). Therefore it follows from Lemma 4.3 that $A \leqslant 0$. Since $\operatorname{Ker} A=\{0\}, A$ is asymptotically stable.

We can now interchange the roles of $A$ and $W$ in (7.1). Since $-W$ is self-adjoint and asymptotically stable, it follows from Theorems 3.1 and 3.2 that $-A$ is a unique solution of the equation

$$
X(-W)+(-W) X=-C^{*} C
$$

and this solution is given by

$$
-A=\int_{0}^{\infty} e^{-t W} C^{*} C e^{-t W} d t
$$

the integral being convergent in the weak operator topology.

However, it is easy to see from (7.2) that $\operatorname{Ker} A=\left(\operatorname{span}\left\{W^{k} c: k \geqslant 0\right\}\right)^{\perp}$ and this subspace is non-trivial since $W$ has multiple spectrum.

Remark. We use the definition of balanced systems with $\operatorname{Ker} W=\{\mathbf{0}\}$. One could think that if we change the definition to admit a non-trivial kernel of $W$, then Theorem 7.1 would not be true anymore. However this is not the case. If we admit $\operatorname{Ker} W \neq\{\mathbf{0}\}$ we can consider the space $\mathcal{K}_{1}=(\operatorname{Ker} W)^{\perp}$. Since

$$
W=\int_{0}^{\infty} e^{t A^{*}} C^{*} C e^{t A} d t=\int_{0}^{\infty} e^{t A} B B^{*} e^{t A^{*}} d t
$$


it follows that

$$
\mathcal{K}_{1}=(\operatorname{Ker} W)^{\perp}=\operatorname{span}\left\{A^{n} b: n \geqslant 0\right\}=\operatorname{span}\left\{\left(A^{*}\right)^{n} c: n \geqslant 0\right\}
$$

so $\mathcal{K}_{1}$ is a reducing subspace for $A$ and $b, c \in \mathcal{K}_{1}$. Therefore if we consider the operators $A_{1}=P_{\mathcal{K}_{1}} A \mid \mathcal{K}_{1}$ and $W_{1}=W \mid \mathcal{K}_{1}$, then $\left(e^{t A} b, c\right)=\left(e^{t A_{1}} b, c\right)$, so the Hankel operator of the systems $\{A, b, c\}$ and $\left\{A_{1}, b, c\right\}$ coincide. But $\operatorname{Ker} W_{1}=\{0\}$ and we arrive at our original definition.

\section{Balanced realizations with discrete time}

The main aim of this chapter is to prove that if a bounded self-adjoint operator satisfies the conditions (C1)-(C3) in the Introduction, then it is unitarily equivalent to a Hankel operator. To construct such a Hankel operator we use linear dynamical systems with discrete time.

The idea of the method can be described briefly as follows. Let $\Gamma$ be a Hankel operator and let $S$ be the shift operator. Then the operator $\Lambda=S^{*} \Gamma$ satisfies the equality

$$
\Gamma^{2}-\Lambda^{2}=(\cdot, p) p
$$

where $p=\Gamma e_{0}$ and $e_{0}$ is the first basis vector in the basis in which $\Gamma$ has a Hankel matrix.

Suppose now that $\Gamma$ is a self-adjoint operator that satisfies the conditions (C1)-(C3). Assume here for simplicity that $\operatorname{Ker} \Gamma=\{0\}$. The problem is to find a vector $p$ in Range $\Gamma$ such that $\Gamma^{2}-(\cdot, p) p \geqslant 0$ and to find a self-adjoint operator $\Lambda$ such that $\Lambda^{2}=\Gamma^{2}-(\cdot, p) p$. Then we can define a contraction $T$ by $\Lambda=T \Gamma$. It is easy to choose a vector $p$ so that $T^{*}$ be an isometry. It can also be seen that $\operatorname{Ker} T$ is one-dimensional. If we could prove that $T^{*}$ is unitarily equivalent to the shift operator, then it would follow from the equality $T \Gamma=\Gamma T^{*}$ that $\Gamma$ is unitarily equivalent to a Hankel operator (see the Introduction). Clearly $T$ is unitarily equivalent to $S^{*}$ if and only if

$$
\left\|T^{n} x\right\| \rightarrow 0
$$

for any vector $x$. However the verification of the above property (the asymptotic stability of $T$ ) is the most difficult problem. We reduce it to the verification of the asymptotic stability of another auxiliary operator. The last problem is much simpler since the auxiliary operator has a sparse spectrum on the unit circle.

In §III.1 we introduce linear dynamical systems with discrete time and associate with each such system a Hankel operator. In §III. 2 for a given operator $\Gamma$ we construct a linear system and we show that if the state space operator $T$ of that system is asymptotically 
stable $\left(T^{*}\right.$ is an isometry if $\left.\operatorname{Ker} \Gamma=\{0\}\right)$ then the Hankel operator associated with the system is unitarily equivalent to $\Gamma$. Such systems are called output normal. As we have already mentioned, it is very difficult to verify the asymptotic stability of $T$. In $\S I I I .3$ we construct another linear system and show that if $T$ is asymptotically stable then it gives another (balanced) realization of the same Hankel operator and we reduce the verification of the asymptotic stability of $T$ to that of the state-space operator of the system constructed. In $\S \S$ III.4-III.9 we choose parameters and prove the asymptotic stability by using the Sz.-Nagy-Foias stability test. Note that in our construction we use essentially the Kato-Rosenblum theorem which claims that the absolutely continuous spectrum is stable under trace class perturbations. In $\S 111.10$ we discuss connections of our results with another theorem in perturbation theory (the Aronszajn-Donoghue theorem) that describes the behavior of the singular spectrum under rank one perturbations.

\section{III.1. Hankel operators and linear systems}

Linear systems with discrete time can be defined by analogy with linear systems with continuous time. Let $\mathcal{K}$ be a Hilbert space (the state space of the system), $B: \mathbf{C} \rightarrow \mathcal{K}$, $C: \mathcal{K} \rightarrow \mathbf{C}$ bounded linear operators,

$$
B u=u b, u \in \mathbf{C}, \quad C x=(x, c), x \in \mathcal{K},
$$

where $b, c \in \mathcal{K}$. Consider the dynamical system $\{A, B, C\}$ with one-dimensional input and one-dimensional output:

$$
\left\{\begin{array}{l}
x_{n+1}=A x_{n}+B u_{n} \\
y_{n}=C x_{n}
\end{array}\right.
$$

In what follows we shall also denote the system (1.1) by $\{A, b, c\}$.

One can associate with the system (1.1) the formal Hankel operator $\Gamma_{\alpha}$ with the matrix

$$
\left\{\alpha_{j+k}\right\}_{j, k \geqslant 0}, \quad \alpha_{j}=\left(A^{j} b, c\right), j \geqslant 0 .
$$

In general $\Gamma_{\alpha}$ need not be bounded on $l^{2}$. However we shall see later that in many important cases it is bounded.

The operator $\Gamma_{\alpha}$ is an analogue of the operator $\Gamma_{h}$ introduced in Chapter II for systems with continuous time. In the case $\sup _{n>0}\left\|A^{n}\right\|<\infty$ it is related to the system (1.1) in the following way. Let $v=\left\{v_{n}\right\}_{n \geqslant 0} \in l^{2}$. Consider the sequence $u=\left\{u_{n}\right\}_{n \in \mathbf{Z}}$ defined by

$$
u_{n}= \begin{cases}v_{-1-n}, & n<0, \\ 0, & n \geqslant 0\end{cases}
$$


Then (as in the case of continuous time) under the initial condition $\lim _{n \rightarrow-\infty} x_{n}=\mathbf{0}$ the output $y$ of the system (1.1) with input $u$ satisfies

$$
y_{n}=\left\{\Gamma_{\alpha} v\right\}_{n}, \quad n \geqslant 0
$$

As in the case of continuous time the system (1.1) is called controllable if $\operatorname{span}\left\{A^{n} b\right.$ : $n \geqslant 0\}=\mathcal{K}$ and observable if $\operatorname{span}\left\{A^{* n} c: n \geqslant 0\right\}=\mathcal{K}$. It is called minimal if it is both controllable and observable.

The controllability and observability Gramians are defined by

$$
\begin{aligned}
& W_{c}=\sum_{j=0}^{\infty} A^{j} B B^{*}\left(A^{*}\right)^{j}, \\
& W_{o}=\sum_{j=0}^{\infty}\left(A^{*}\right)^{j} C^{*} C A^{j}
\end{aligned}
$$

if the series converge in the weak operator topology. In this case the system is controllable (observable) if and only if $\operatorname{Ker} W_{c}=\{0\}\left(\operatorname{Ker} W_{o}=\{0\}\right)$.

A minimal system is called balanced if the series (1.2)-(1.3) converge and $W_{c}=W_{o}$. We shall also consider the so-called output normal systems, i.e. the systems for which the series (1.2)-(1.3) converge and $W_{o}=I$.

The main result of this chapter is the following theorem which together with Theorem 1.1 of Chapter I gives a complete solution of the problem of the description of self-adjoint operators which are unitarily equivalent to a Hankel operator and proves Theorem 1 in the Introduction.

THEOREM 1.1. Let $\Gamma$ be a self-adjoint operator on Hilbert space which satisfies the conditions $(\mathrm{C} 1),(\mathrm{C} 2)$, and $(\mathrm{C} 3)$ in the Introduction. Then there exists a balanced system $\{A, b, c\}$ with one-dimensional output and one-dimensional input such that the Hankel operator $\Gamma_{\alpha}$ associated with the system is unitarily equivalent to $\Gamma$.

The proof of Theorem 1.1 will be given in $§ I I I .8$.

\section{III.2. Output normal realizations of Hankel operators}

In this section for a given self-adjoint operator we construct a linear system and prove that if the state space operator is asymptotically stable then the Hankel operator associated with the system is unitarily equivalent to the operator in question. Moreover the system constructed is output normal. The main difficulty however consists in the verification of the asymptotic stability. 
Let $R$ be a self-adjoint operator on a Hilbert space $\mathcal{K}$ with trivial kernel and let $q$ be a vector in $\mathcal{K}$ such that $\|q\| \leqslant 1$. Then $R^{2}-(\cdot, p) p \geqslant 0$, where $p=R q$. Let $\Lambda$ be a self-adjoint operator such that

$$
R^{2}=\Lambda^{2}+(\cdot, p) p
$$

Clearly $\Lambda^{2} \leqslant R^{2}$ and so by the Douglas lemma (see [D1]) there exists a contraction $T$ (which is unique because of the fact that $\operatorname{Ker} R=\{0\}$ ) such that $T R=\Lambda$.

Consider now the dynamical system $\{T, p, q\}$. As above $\alpha_{j}=\left(T^{j} p, q\right), j \geqslant 0$, and $\Gamma_{\alpha}$ is the Hankel operator with matrix $\left\{\alpha_{j+k}\right\}_{j, k} \geqslant 0$.

We shall call $T$ asymptotically stable if $\left\|T^{n} x\right\| \rightarrow 0$ as $n \rightarrow \infty$ for any $x \in \mathcal{K}$.

THEOREM 2.1. Suppose that $T$ is asymptotically stable. Then

(i) the system $\{T, p, q\}$ is output normal;

(ii) $R$ is unitarily equivalent to $\Gamma_{\alpha} \mid\left(\operatorname{Ker} \Gamma_{\alpha}\right)^{\perp}$;

(iii) $\operatorname{Ker} \Gamma_{\alpha}=\{\mathbf{0}\}$ if and only if $\|q\|=1$ and $q \notin$ Range $R$.

Proof. It follows from (2.1) and the definition of $T$ that

$$
R^{2}=R T^{*} T R+(\cdot, R q) R q
$$

and since Ker $R=\{0\}$, we obtain

$$
I=T^{*} T+(\cdot, q) q
$$

Therefore

$$
\|x\|^{2}=\|T x\|^{2}+|(x, q)|^{2}, \quad x \in \mathcal{K} .
$$

If we apply (2.3) to $T x$ and use the fact that $T$ is asymptotically stable, we obtain

$$
\|x\|^{2}=\sum_{j=0}^{\infty}\left|\left(T^{j} x, q\right)\right|^{2}, \quad x \in \mathcal{K}
$$

which means that the operator $V: \mathcal{K} \rightarrow l^{2}$ defined by

$$
V x=\left((x, q),(T x, q),\left(T^{2} x, q\right), \ldots\right)
$$

is an isometry. It is easy to see that the Hankel operator $\Gamma_{\alpha}$ associated with the system satisfies the equality $\Gamma_{\alpha}=V R V^{*}$. Indeed

$$
V^{*}\left\{y_{j}\right\}_{j \geqslant 0}=\sum_{j \geqslant 0} y_{j}\left(T^{*}\right)^{j} q, \quad\left\{y_{j}\right\}_{j \geqslant 0} \in l^{2} .
$$


So if $\left\{e_{j}\right\}_{j \geqslant 0}$ is the standard orthonormal basis of $l^{2}$ then

$$
\left(V R V^{*} e_{j}, e_{k}\right)=\left(R\left(T^{*}\right)^{j} q,\left(T^{*}\right)^{k} q\right)
$$

and since $R T^{*}=T R$ (this follows from the definition of $T$ ), we have

$$
\left(V R V^{*} e_{j}, e_{k}\right)=\left(T^{j} R q,\left(T^{*}\right)^{k} q\right)=\left(T^{j+k} p, q\right)=\alpha_{j+k}
$$

Therefore (ii) holds and $\operatorname{Ker} \Gamma_{\alpha}=\operatorname{Ker} V^{*}$.

It is clear from (2.4) and (2.5) that the series (1.3) converges in the weak operator topology and $W_{o}=V^{*} V=I$. On the other hand, since $T R=R T^{*}=\Lambda$, we have

$$
\begin{aligned}
W_{c} & =\sum_{j \geqslant 0} T^{j}((\cdot, R q) R q)\left(T^{*}\right)^{j}=\sum_{j \geqslant 0} T^{j} R((\cdot, q) q) R\left(T^{*}\right)^{j} \\
& =\sum_{j \geqslant 0} R\left(T^{*}\right)^{j}((\cdot, q) q) T^{j} R=R W_{o} R=R^{2}
\end{aligned}
$$

and so the series (1.2) converges in the weak operator topology.

Note that $\operatorname{Ker} \Gamma_{\alpha}=\{0\}$ if and only if $V$ is onto which is equivalent to the fact that $\left\{\left(T^{*}\right)^{j} q\right\}_{j \geqslant 0}$ is an orthonormal basis in $\mathcal{K}$.

Suppose now that $\|q\|=1$ and $q \notin$ Range $R$. We have by the definition of $\Lambda$

$$
\Lambda^{2}=R(I-(\cdot, q) q) R
$$

Since $\operatorname{Ker} R=\{0\}$, it is clear that $\operatorname{Ker} \Lambda=\{0\}$. It follows now from the equality $R T^{*}=\Lambda$ that $\operatorname{Ker} T^{*}=\{0\}$.

Applying (2.2) to the vector $q$, we obtain

$$
T^{*} T q+q=q
$$

and since $\operatorname{Ker} T^{*}=\{0\}$, it follows that $T q=0$.

Multiplying (2.2) by $T$ on the left and by $T^{*}$ on the right, we obtain

$$
T T^{*} T T^{*}+T((\cdot, q) q) T^{*}=T T^{*}
$$

and since $T q=0$, we have $\left(T T^{*}\right)^{2}=T T^{*}$. Clearly in view of the fact $\operatorname{Ker} T^{*}=\{0\}$ this means that $T T^{*}=I$, i.e. $T^{*}$ is an isometry. Since $T$ is asymptotically stable, $T$ has no unitary part. The condition (2.2) means that $T^{*}$ has defect 1 and so $T^{*}$ is unitarily equivalent to the shift operator $S$. Therefore the conditions $T q=0$ and $\|q\|=1$ imply that the system $\left\{\left(T^{*}\right)^{j} q\right\}_{j \geqslant 0}$ forms an orthonormal basis in $\mathcal{K}$. 
Suppose now that $\left\{\left(T^{*}\right)^{j} q\right\}_{j \geqslant 0}$ is an orthonormal basis in $\mathcal{K}$. Then $\|q\|=1$ and $T^{*}$ is an isometry, i.e. $T T^{*}=I$. Therefore we have from $(2.7)$

$$
T((\cdot, q) q) T^{*}=(\cdot, T q) T q=\mathbf{0}
$$

and so $T q=0$. Since $T^{*}$ and $R$ have trivial kernels, the operator $\Lambda=R T^{*}$ also has trivial kernel. But it is easy to see that (2.6) and the fact that $\|q\|=1$ imply that $\operatorname{Ker} \Lambda=\{0\}$ if and only if $q \notin$ Range $R$.

Remark. As we have shown in the proof, in the case $\operatorname{Ker} \Gamma=\{0\}$ the operator $T$ is unitarily equivalent to the backward shift $S^{*}$. It is easy to see that otherwise $T$ is unitarily equivalent to the restriction of $S^{*}$ to its invariant subspace $\left(\operatorname{Ker} \Gamma_{\alpha}\right)^{\perp}$.

Theorem 2.1 gives us a recipe how to construct a Hankel operator which is unitarily equivalent to our operator $\Gamma$. We can put $R=\Gamma \mid(\operatorname{Ker} \Gamma)^{\perp}$. Then we have to choose a vector $q$ such that $R^{2}-(\cdot, R q) R q \geqslant 0$. If $\operatorname{Ker} \Gamma=\{\mathbf{0}\}$, then $q$ must satisfy the conditions $\|q\|=1$ and $q \notin$ Range $R$. If $\operatorname{Ker} \Gamma \neq\{0\}$, at least one of the conditions must be violated. Next, we have to choose a self-adjoint operator $\Lambda$ such that $\Lambda^{2}=R^{2}-(\cdot, R q) R q$. The problem will be solved if we manage to prove that the operator $T$ uniquely defined by the equality $T R=\Lambda$ is asymptotically stable. However this is the most difficult point.

\section{III.3. Balanced realizations}

In this section we construct a balanced system and show that the verification of the asymptotic stability of $T$ can be reduced to the verification of the asymptotic stability of the adjoint of the state space operator of the balanced system constructed. It turns out that the last problem is considerably simpler.

LEMMA 3.1. Let $T$ be a contraction and let $K, X$ be bounded operators on Hilbert space such that $X$ has dense range and

$$
T X=X K
$$

If $K$ is asymptotically stable, then so is $T$.

Proof. The result follows immediately from the formula $T^{j} X=X K^{j}, j \geqslant 1$, and from the facts that $\|T\| \leqslant 1$ and $X$ has dense range.

Let $T, \Lambda, R$ be the operators considered in $\S I I I .2$. Put $W=|R|=\left(R^{*} R\right)^{1 / 2}$. 
LEMMA 3.2. There exists a unique contraction $A$ such that

$$
A W^{1 / 2}=W^{1 / 2} T^{*}
$$

Proof. The inequality $T^{*} T \leqslant I$ implies

$$
W^{2}=R^{2} \geqslant R T^{*} T R=\Lambda^{2}=T R^{2} T^{*}=T W^{2} T^{*} \geqslant T W T^{*} T W T^{*} .
$$

By the Heinz inequality (see [BS1, Chapter 10.4]) this implies that $T W T^{*} \leqslant W$. Applying now the Douglas lemma (see [D1]) we find that there exists a unique contraction $A$ satisfying (3.1).

It follows from (3.1) that

$$
T W^{1 / 2}=W^{1 / 2} A^{*}
$$

and so by Lemma 3.1 to prove that $T$ is asymptotically stable, it is sufficient to show that so is $A^{*}$.

Let $\boldsymbol{J}$ be the "argument" of $R$, i.e.

$$
R=\boldsymbol{J}|R|=\boldsymbol{J} W=W \boldsymbol{J}, \quad \boldsymbol{J}=\boldsymbol{J}^{*}=\boldsymbol{J}^{-1} .
$$

Consider now the dynamical system $\{A, b, c\}$ where the operator $A$ is defined in Lemma $3.2, b=W^{1 / 2} q, c=J W^{1 / 2} q$.

Although the following theorem will not be used in the proof of Theorem 1.1, it is of independent interest and establishes relations between balanced realizations and output normal ones.

ThEOREM 3.3. Let $T$ be asymptotically stable. Then the system $\{A, b, c\}$ is a balanced realization of the Hankel operator associated with the output normal system $\{T, p, q\}$.

In other words the system $\{A, b, c\}$ is balanced and $\left(A^{j} b, c\right)=\left(T^{j} p, q\right), j \geqslant 0$. To prove Theorem 3.3 we need the following lemma.

LEMMA 3.4. $A^{*} \boldsymbol{J}=\boldsymbol{J} A$.

Proof. Since $\operatorname{Ker} W=\{\mathbf{0}\}$ and $W$ has dense range, it is sufficient to show that $W^{1 / 2} A^{*} J W^{1 / 2}=W^{1 / 2} J A W^{1 / 2}$. We have by (3.1)

$$
W^{1 / 2} A^{*} J W^{1 / 2}=T W^{1 / 2} J W^{1 / 2}=T J W=T R=\Lambda
$$

by the definition of $T$. On the other hand, by (3.1)

$$
W^{1 / 2} J A W^{1 / 2}=W^{1 / 2} J W^{1 / 2} T^{*}=J W T^{*}=R T^{*}=\Lambda,
$$


which proves the result.

Proof of Theorem 3.3. Let us first show that $\{A, b, c\}$ is a balanced system. We have

$$
\begin{aligned}
W_{c} & =\sum_{j \geqslant 0} A^{j}\left(\left(\cdot, W^{1 / 2} q\right) W^{1 / 2} q\right)\left(A^{*}\right)^{j} \\
& =\sum_{j \geqslant 0} A^{j} W^{1 / 2}((\cdot, q) q) W^{1 / 2}\left(A^{*}\right)^{j} \\
& =\sum_{j \geqslant 0} W^{1 / 2}\left(T^{*}\right)^{j}((\cdot, q), q) T^{j} W^{1 / 2}
\end{aligned}
$$

by $(3.1)$. Hence

$$
W_{c}=W^{1 / 2}\left(\sum_{j \geqslant 0}\left(\cdot,\left(T^{*}\right)^{j} q\right)\left(T^{*}\right)^{j} q\right) W^{1 / 2}=W,
$$

since the operator $V$ defined by (2.4) is an isometry. Clearly the series (1.2) converges in the weak operator topology. Next

$$
\begin{aligned}
W_{o} & =\sum_{j \geqslant 0}\left(A^{*}\right)^{j}\left(\left(\cdot, J W^{1 / 2} q\right) J W^{1 / 2} q\right) A^{j} \\
& =\sum_{j \geqslant 0}\left(A^{*}\right)^{j} J W^{1 / 2}((\cdot, q) q) W^{1 / 2} J A^{j} \\
& =\sum_{j \geqslant 0} J A^{j}\left(\left(\cdot, W^{1 / 2} q\right) W^{1 / 2} q\right)\left(A^{*}\right)^{j} J
\end{aligned}
$$

by Lemma 3.4. Hence

$$
W_{o}=\boldsymbol{J} W_{c} \boldsymbol{J}=\boldsymbol{J} W \boldsymbol{J}=W
$$

and the series (1.3) converges in the weak operator topology.

Let us now prove the equality $\left(A^{j} b, c\right)=\left(T^{j} p, f\right), j \geqslant 0$. Bearing in mind (3.1) and the equality $R T^{*}=T R$, we have

$$
\begin{aligned}
\left(A^{j} b, c\right) & =\left(A^{j} W^{1 / 2} q, J W^{1 / 2} q\right)=\left(W^{1 / 2} J A^{j} W^{1 / 2} q, q\right) \\
& =\left(W^{1 / 2} J W^{1 / 2}\left(T^{*}\right)^{j} q, q\right)=\left(R\left(T^{*}\right)^{j} q, q\right)=\left(T^{j} R q, q\right)=\left(T^{j} p, q\right) .
\end{aligned}
$$

Let $\mathcal{J}$ be the "argument" of $\Lambda$, i.e.

$$
\Lambda=\mathcal{J}|\Lambda|=|\Lambda| \mathcal{J}, \quad \mathcal{J}=\mathcal{J}^{*}=\mathcal{J}^{-1} .
$$

If $\operatorname{Ker} \Lambda \neq\{0\}$, then $\mathcal{J}$ is not uniquely defined, so by $\mathcal{J}$ we understand an arbitrary operator which satisfies (3.2).

Note that $W^{2}=R^{2} \geqslant \Lambda^{2}$, and so by the Heintz inequality (see [BS1, §10.4]), $W \geqslant|\Lambda|$. Hence by the Douglas lemma [D1] there exists a unique contraction $Q$ such that

$$
Q W^{1 / 2}=|\Lambda|^{1 / 2} \text {. }
$$


LEMMA 3.5. $A^{*}=Q^{*} \mathcal{J} Q J$.

Proof. We have by (3.1)

$$
W^{1 / 2} A^{*} J W^{1 / 2}=T W^{1 / 2} J W^{1 / 2}=T R=\Lambda .
$$

On the other hand,

$$
\Lambda=|\Lambda|^{1 / 2} \mathcal{J}|\Lambda|^{1 / 2}=W^{1 / 2} Q^{*} \mathcal{J} Q W^{1 / 2}
$$

So

$$
W^{1 / 2} A^{*} J W^{1 / 2}=W^{1 / 2} Q^{*} \mathcal{J} Q W^{1 / 2} .
$$

Since $\operatorname{Ker} W^{1 / 2}=\{\mathbf{0}\}$, we have $A^{*} J=Q^{*} \mathcal{J} Q$ which completes the proof.

The following lemma describes the structure of $Q$.

Lemma 3.6. Let $\mathcal{K}_{0}$ be the smallest invariant subspace of $W$ that contains $J q$. Then $Q$ has the following structure in the decomposition $\mathcal{K}=\mathcal{K}_{0} \oplus \mathcal{K}_{0}^{\perp}$ :

$$
\left(\begin{array}{cc}
Q_{0} & \mathbf{0} \\
\mathbf{0} & I
\end{array}\right)
$$

where $Q_{0}$ is a pure contraction (i.e. $\left\|Q_{0} h\right\|<\|h\|$ for $h \neq 0$ ).

Proof. Clearly $W^{2}$ and $\Lambda^{2}$ coincide on $\mathcal{K}_{0}^{\perp}$ and $\mathcal{K}_{0}^{\perp}$ is a reducing subspace for both of them. Therefore the same is true for the operators $W^{1 / 2}$ and $|\Lambda|^{1 / 2}$ which together with (3.3) proves (3.4). It remains to show that $Q_{0}$ is a pure contraction.

By (3.3)

$$
W^{1 / 2} Q^{*} Q W Q^{*} Q W^{1 / 2}=\Lambda^{2}=W^{2}-(\cdot, p) p=W^{1 / 2}\left(W-\left(\cdot, W^{1 / 2} J q\right) W^{1 / 2} J q\right) W^{1 / 2}
$$

and so

$$
Q^{*} Q W Q^{*} Q=W-\left(\cdot, W^{1 / 2} J q\right) W^{1 / 2} J q .
$$

It follows from (3.5) that

$$
\left(W Q^{*} Q x, Q^{*} Q x\right)=(W x, x)+\left|\left(x, W^{1 / 2} J_{q}\right)\right|^{2}, \quad x \in \mathcal{H} .
$$

Since $Q$ is a contraction, $\|Q h\|=\|h\|$ if and only if $Q^{*} Q h=h$ (i.e. $h \in \operatorname{Ker}\left(I-Q^{*} Q\right)$ ). It follows from (3.6) that if $h \in \operatorname{Ker}\left(I-Q^{*} Q\right)$ then $h \perp W^{1 / 2} J q$ and by (3.5) we obtain $Q^{*} Q W h=W h$. So $\operatorname{Ker}\left(I-Q^{*} Q\right)$ is an invariant subspace of $W$ which is orthogonal to $W^{1 / 2} J q$. Therefore $\operatorname{Ker}\left(I-Q^{*} Q\right)$ is orthogonal to $\mathcal{K}_{0}$ which proves that $Q_{0}$ is a pure contraction. 


\section{III.4. Asymptotic stability}

The material of the previous section gives us a clear way to construct a Hankel operator which is unitarily equivalent to a given operator $\Gamma$. Let $\mathcal{K}=(\operatorname{Ker} \Gamma)^{\perp}, \widetilde{\Gamma}=\Gamma \mid \mathcal{K}$, and $W=|\widetilde{\Gamma}|$. We have to choose a vector $q$, operators $J$ and $\mathcal{J}$ such that the following properties hold:

(i) $\boldsymbol{J}=\boldsymbol{J}^{*}=\boldsymbol{J}^{-1}, \boldsymbol{J} W=W \boldsymbol{J}$ and $R \stackrel{\text { def }}{=} \boldsymbol{J W}$ is unitarily equivalent to $\widetilde{\Gamma}$;

(ii) $R^{2}-(\cdot, R q) R q \geqslant 0$;

(iii) $\mathcal{J}=\mathcal{J}^{*}=\mathcal{J}^{-1}, \mathcal{J}\left(R^{2}-(\cdot, R q) R q\right)^{1 / 2}=\left(R^{2}-(\cdot, R q) R q\right)^{1 / 2} \mathcal{J}$;

(iv) the operator $A^{*}=Q^{*} \mathcal{J} Q J$ is asymptotically stable, where the contraction $Q$ is uniquely defined by (3.3) and

$$
\Lambda=\mathcal{J}\left(R^{2}-(\cdot, R q) R q\right)^{1 / 2}
$$

If $\operatorname{Ker} \Gamma=\{\mathbf{0}\}$, we have to impose the following condition:

(v) $\|q\|=1$ and $q \notin$ Range $R$.

If $\operatorname{Ker} \Gamma \neq\{0\}$, we have to make the assumption:

$\left(\mathrm{v}^{\prime}\right)$ either $\|q\|<1$ or $q \in$ Range $R$.

The most difficult problem here is to verify the asymptotic stability of $A^{*}$. To this end we shall use the following stability test which is an immediate consequence of Proposition 6.7 in Chapter II of [SF] (in Chapter II of our paper we have used an analogue of this test for semigroups of contractions).

Stability test. Let $\boldsymbol{T}$ be a contraction on Hilbert space. If $\boldsymbol{T}$ has no eigenvalues on the unit circle $\mathbf{T}$ and the set $\sigma(\boldsymbol{T}) \cap \mathbf{T}$ is at most countable, then $\boldsymbol{T}$ is asymptotically stable.

We define the Fredholm spectrum $\sigma_{F}(\boldsymbol{T})$ of a bounded linear operator $\boldsymbol{T}$ (see [N]) as the set of points $\lambda$ in $\mathbf{C}$ for which there exists a sequence $\left\{x_{n}\right\}_{n \geqslant 1}$ such that $\left\|x_{n}\right\|=1$, w- $\lim _{n \rightarrow \infty} x_{n}=\mathbf{0}$ (i.e. $\lim _{n \rightarrow \infty} x_{n}=\mathbf{0}$ in the weak topology), and

$$
\lim _{n \rightarrow \infty}\left\|\boldsymbol{T} x_{n}-\lambda x_{n}\right\|=0
$$

Clearly it is sufficient to prove that $\sigma_{F}\left(A^{*}\right) \cap \mathbf{T}$ is at most countable and both $A$ and $A^{*}$ do not have eigenvalues on $\mathbf{T}$. It follows easily from Lemma 3.5 that $A^{*}=\boldsymbol{J} A \boldsymbol{J}$ and so the operators $A$ and $A^{*}$ are unitarily equivalent. Therefore it is sufficient to check that $A^{*}$ has no eigenvalues on $\mathbf{T}$ (and certainly that $\sigma_{F}\left(A^{*}\right) \cap \mathbf{T}$ is at most countable). The following lemma will help us to get rid of eigenvalues on $\mathbf{T}$. Recall that $\mathcal{K}_{0}$ is the smallest $W$-invariant subspace of $\mathcal{K}$ that contains $\boldsymbol{J} q$. 
Lemma 4.1. Let $|\zeta|=1$ and $h \in \mathcal{H}$. Then $A^{*} h=\zeta h$ if and only if $h, \boldsymbol{J} h \in \mathcal{K}_{0}^{\perp}$ and $\mathcal{J} J h=\zeta h$.

Proof. Suppose that $A^{*} h=\zeta h,|\zeta|=1$. Since $A^{*}=Q^{*} \mathcal{J} Q J$ and $Q$ is a contraction, it follows that $\|Q \boldsymbol{J} h\|=\|\boldsymbol{J} h\|=\|h\|$.

By Lemma 3.6, $\boldsymbol{J} h \in \mathcal{K}_{0}^{\perp}$ and $Q \boldsymbol{J} h=\boldsymbol{J} h$. Next, the equality $\left\|A^{*} h\right\|=\|h\|$ implies $\left\|Q^{*} \mathcal{J} Q \boldsymbol{J} h\right\|=\left\|Q^{*} \mathcal{J} J h\right\|=\|\mathcal{J} \boldsymbol{J} h\|$. So by Lemma 3.6, $Q^{*} \mathcal{J} Q \boldsymbol{J} h=Q^{*} \mathcal{J} \boldsymbol{J} h=\mathcal{J} \boldsymbol{J} h$. Hence $\mathcal{J} \boldsymbol{J} h=\zeta h$ and $h \in \mathcal{K}_{0}^{\perp}$.

The converse is trivial. If $\boldsymbol{J} h \in \mathcal{K}_{0}^{\frac{1}{0}}$, by Lemma 3.6, $\mathcal{J} Q \boldsymbol{J} h=\mathcal{J} \boldsymbol{J} h=\zeta h$. Since $h \in \mathcal{K}_{0}^{\frac{1}{0}}$, it follows from Lemma 3.6 that $A^{*} h=Q^{*} \mathcal{J} Q \boldsymbol{J} h=\zeta h$.

The following lemma will be used to estimate $\sigma_{F}\left(A^{*}\right) \cap \mathbf{T}$.

Lemma 4.2. Suppose that

$$
\lim _{n \rightarrow \infty}\left\|\left(Q^{*} \mathcal{J} Q-\mathcal{J}\right) g_{n}\right\|=0
$$

for any sequence $\left\{g_{n}\right\}_{n \geqslant 1}$ such that $\left\|g_{n}\right\|=1, \lim _{n \rightarrow \infty}\left\|Q g_{n}\right\|=1$, and $\mathrm{w}-\lim _{n \rightarrow \infty} g_{n}=\mathbf{0}$. Then $\sigma_{F}\left(A^{*}\right) \cap \mathbf{T} \subset \sigma_{F}(\mathcal{J J})$.

Proof. Let $\zeta \in \sigma_{F}\left(A^{*}\right)$. Then there exists a sequence $\left\{x_{n}\right\}_{n \geqslant 1}$ such that $\left\|x_{n}\right\|=1$, w$\lim _{n \rightarrow \infty} x_{n}=0$, and $\lim _{n \rightarrow \infty}\left\|A^{*} x_{n}-\eta x_{n}\right\|=0$. Let $g_{n}=J x_{n}$. Then clearly $\left\|g_{n}\right\|=1$ and $\mathrm{w}-\lim _{n \rightarrow \infty} g_{n}=\mathbf{0}$. We have

$$
\left\|A^{*} x_{n}-\zeta x_{n}\right\|=\left\|Q^{*} \mathcal{J} Q g_{n}-\zeta x_{n}\right\| \rightarrow 0 .
$$

Since $|\zeta|=1$, it follows that $\left\|Q g_{n}\right\| \rightarrow 1$. Therefore by the hypotheses

$$
\left\|A^{*} x_{n}-\mathcal{J J} x_{n}\right\|=\left\|Q^{*} \mathcal{J} Q g_{n}-\mathcal{J} g_{n}\right\| \rightarrow 0,
$$

which together with (4.1) yields $\left\|\mathcal{J} J x_{n}-\zeta x_{n}\right\| \rightarrow 0$. So $\zeta \in \sigma_{F}(\mathcal{J J})$.

\section{III.5. The main construction}

The operator $W$ admits a representation as multiplication by $t$ on the direct integral

$$
\mathcal{K}=\int_{\sigma(W)} \oplus E(t) d \varrho(t)
$$

where $\varrho$ is a spectral measure of $W$. Let $\nu_{W}(t) \stackrel{\text { def }}{=} \operatorname{dim} E(t)$ be the spectral multiplicity function of $W$. As we have discussed in the Introduction we can assume that all spaces $E(t)$ are imbedded in a Hilbert space $E$ with an orthonormal basis $\left\{e_{j}\right\}_{j \geqslant 1}$ and

$$
E(t)=\operatorname{span}\left\{e_{j}: 1 \leqslant j<\nu_{W}(t)+1\right\} .
$$


Recall that $\nu$ is the spectral multiplicity function of $\Gamma$. Clearly $\nu(t)=\nu_{R}(t)$ for $t \neq 0$, where $\nu_{R}$ is the multiplicity function of $R$. Clearly $\nu_{W}(t)=\nu_{R}(t)+\nu_{R}(-t), t>0$. Note that we have not yet defined the operator $R$. But since $R$ must be unitarily equivalent to $\widetilde{\Gamma}=\Gamma \mid(\operatorname{Ker} \Gamma)^{\perp}$, the above equalities must be satisfied.

We split the set $\sigma(W)$ in five disjoint subsets:

$$
\begin{aligned}
\sigma_{0} & =\left\{t \in \sigma(W): \nu_{R}(t)=\nu_{R}(-t)\right\}, \\
\sigma_{1}^{(+)} & =\left\{t \in \sigma(W): \nu_{R}(t)=\nu_{R}(-t)+1\right\}, \\
\sigma_{1}^{(-)} & =\left\{t \in \sigma(W): \nu_{R}(t)=\nu_{R}(-t)-1\right\}, \\
\sigma_{2}^{(+)} & =\left\{t \in \sigma(W): \nu_{R}(t)=\nu_{R}(-t)+2\right\}, \\
\sigma_{2}^{(-)} & =\left\{t \in \sigma(W): \nu_{R}(t)=\nu_{R}(-t)-2\right\} .
\end{aligned}
$$

Since $\Gamma$ satisfies the condition (C3), it follows that $\sigma_{0} \cup \sigma_{1}^{(+)} \cup \sigma_{1}^{(-)} \cup \sigma_{2}^{(+)} \cup \sigma_{2}^{(-)}=\sigma(W)$.

We choose the operator $J$ to be multiplication by the operator-valued function $J$ which is defined as follows. For $t \in \sigma_{0}$ we put

$$
J(t)=\left(\begin{array}{ccccc}
0 & 1 & 0 & 0 & \ldots \\
1 & 0 & 0 & 0 & \ldots \\
0 & 0 & 0 & 1 & \ldots \\
0 & 0 & 1 & 0 & \ldots \\
\vdots & \vdots & \vdots & \vdots & \ddots
\end{array}\right) .
$$

For $t \in \sigma_{1}^{( \pm)}$we put

$$
J(t)=\left(\begin{array}{cccccc}
0 & 1 & \ldots & 0 & 0 & 0 \\
1 & 0 & \ldots & 0 & 0 & 0 \\
\vdots & \vdots & \ddots & \vdots & \vdots & \vdots \\
0 & 0 & \ldots & 0 & 1 & 0 \\
0 & 0 & \ldots & 1 & 0 & 0 \\
0 & 0 & \ldots & 0 & 0 & \pm 1
\end{array}\right)
$$

(we assume that $J(t)= \pm 1$ if $\nu_{W}=1$ ). Finally for $t \in \sigma_{2}^{( \pm)}$we put

$$
J(t)=\left(\begin{array}{ccccccc} 
\pm 1 & 0 & 0 & \ldots & 0 & 0 & 0 \\
0 & 0 & 1 & \ldots & 0 & 0 & 0 \\
0 & 1 & 0 & \ldots & 0 & 0 & 0 \\
\vdots & \vdots & \vdots & \ddots & \vdots & \vdots & \vdots \\
0 & 0 & 0 & \ldots & 0 & 1 & 0 \\
0 & 0 & 0 & \ldots & 1 & 0 & 0 \\
0 & 0 & 0 & \ldots & 0 & 0 & \pm 1
\end{array}\right)
$$


(we assume that

$$
J(t)= \pm\left(\begin{array}{ll}
1 & 0 \\
0 & 1
\end{array}\right)
$$

if $\left.\nu_{W}(t)=2\right)$.

Now we can define $R$ by $R=J W$.

Denote by $\mathcal{K}_{0}$ the "first level" of $\mathcal{K}$, i.e.

$$
\mathcal{K}_{0}=\left\{f \in \mathcal{K}: f(t)=\varphi(t) e_{1}, \varphi \in L^{2}(\varrho)\right\} .
$$

Clearly $\mathcal{K}_{0}$ can naturally be identified with $L^{2}(\varrho)$.

Let now $q_{0}$ be a vector in $\mathcal{K}_{0}$ that satisfies the following properties:

(1) $\left\|q_{0}\right\|=1$;

(2) $q_{0}$ is a cyclic vector of $W \mid \mathcal{K}_{0}$ (in other words $q_{0}(t) \neq 0, \varrho$-a.e.);

(3) $q_{0} \in$ Range $W$ if $\operatorname{Ker} \Gamma \neq\{0\}$ and $q_{0} \notin$ Range $W$ if $\operatorname{Ker} \Gamma=\{0\}$ (in the last case $W$ is non-invertible and so such a $q_{0}$ exists).

We define the vector $q$ by $q=J q_{0}$, so $q_{0}=J q$ and the definition of $\mathcal{K}_{0}$ is consistent with that given in Lemma 3.6. Let $p=R q=R J q_{0}=W q_{0}$ and $\Lambda^{2}=W^{2}-(\cdot, p) p$. (Note that we do not define the operator $\Lambda$, we define only the operator $\Lambda^{2}$ and to define $\Lambda$ we have to choose a reasonable square root of $\Lambda^{2}$ which will be done later.) Clearly

$$
\left(W^{2} x, x\right)-(x, p)(p, x)=\|W x\|^{2}-|(x, R q)|^{2} \geqslant\|W x\|^{2}-\|R x\|^{2}\|q\|^{2}=0,
$$

since $\|R x\|=\|W x\|$ and $\|q\|=1$. So $W^{2}-(\cdot, p) p \geqslant 0$. Obviously $\mathcal{K}_{0}$ is a reducing subspace for both $W^{2}$ and $\Lambda^{2}$, and $W$ and $|\Lambda| \stackrel{\text { def }}{=}\left(\Lambda^{2}\right)^{1 / 2}$ coincide on $\mathcal{K}_{0}^{\perp}$.

By the Kato-Rosenblum theorem (see [Ka, Chapter 10]) the absolutely continuous parts of $W^{2}$ and $\Lambda^{2}$ are unitarily equivalent. So if $\lambda$ is a scalar spectral measure of $|\Lambda| \mid \mathcal{K}_{0}$, then the absolutely continuous components $\varrho_{a}$ and $\lambda_{a}$ of the measures $\varrho$ and $\lambda$ are mutually absolutely continuous. Therefore there are disjoint Borel sets $\Delta, \delta_{\varrho}$ and $\delta_{\lambda}$ such that $\varrho$ and $\lambda$ are mutually absolutely continuous on $\Delta, \varrho$ is supported on $\delta_{\varrho} \cup \Delta, \lambda$ is supported on $\delta_{\lambda} \cup \Delta$, and the sets $\delta_{\varrho}$ and $\delta_{\lambda}$ have zero Lebesgue measure. Clearly we can assume that $\varrho$ and $\lambda$ coincide on $\Delta$. Put $\tilde{\varrho}=\varrho+\lambda \mid \delta_{\lambda}$. Consider the von Neumann integral

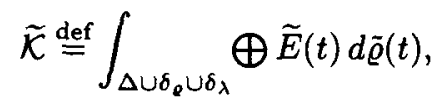

where $\widetilde{E}(t)=\operatorname{span}\left\{e_{k}: 1 \leqslant k<\nu_{W}(t)+1\right\}$ for $t \in \Delta, \widetilde{E}(t)=\operatorname{span}\left\{e_{k}: 2 \leqslant k<\nu_{W}(t)+1\right\}$ for $t \in \delta_{Q}$, and $\widetilde{E}(t)=\left\{\zeta e_{1}: \zeta \in \mathbf{C}\right\}$ for $t \in \delta_{\lambda}$.

It is easy to see that $p$ is a cyclic vector of $\Lambda^{2} \mid \mathcal{K}_{0}$ and so $|\Lambda| \mid \mathcal{K}_{0}$ is a cyclic operator. Therefore there exists a unitary operator $U_{0}$ from $L^{2}(\varrho)$ onto $L^{2}(\lambda)$ such that $U_{0}\left(|\Lambda| \mid \mathcal{K}_{0}\right) U_{0}^{*}$ is multiplication by the independent variable on $L^{2}(\lambda)$. 
The operator $|\Lambda|$ can be realized as multiplication on $\widetilde{\mathcal{K}}$. Namely if we define the unitary operator $U: \mathcal{K}=L^{2}(\varrho) \oplus \mathcal{K}_{0}^{\perp} \rightarrow L^{2}(\lambda) \oplus \mathcal{K}_{0}^{\perp}$ by the operator matrix

$$
U=\left(\begin{array}{cc}
U_{0} & \mathbf{0} \\
\mathbf{0} & I
\end{array}\right)
$$

then $|\Lambda|=U^{*} M_{t} U$, where $M_{t}$ is multiplication by $t$ on $\widetilde{\mathcal{K}}$. (Note that $|\Lambda|$ coincides with $W$ on $\mathcal{K}_{0}^{+}$.)

We define now the operator $\mathcal{J}$ (the argument of $\Lambda$ ) as $\mathcal{J}=U^{*} \widetilde{\mathcal{J}} U$, where $\widetilde{\mathcal{J}}$ is multiplication by an operator-valued function $\widetilde{\mathcal{J}}$ of the following form.

If $t \in \delta_{\lambda}$, we put

$$
\tilde{\mathcal{J}}(t)=1
$$

(in this case $\operatorname{dim} \widetilde{E}(t)=1$ ). If $t \in \sigma_{2}^{( \pm)}$(in this case $t \in \Delta$ by (C3)) we put

$$
\widetilde{\mathcal{J}}(t)=\left(\begin{array}{ccccc}
\cos \omega_{1}(t) & \sin \omega_{1}(t) & 0 & 0 & \ldots \\
\sin \omega_{1}(t) & -\cos \omega_{1}(t) & 0 & 0 & \ldots \\
0 & 0 & \cos \omega_{2}(t) & \sin \omega_{2}(t) & \ldots \\
0 & 0 & \sin \omega_{2}(t) & -\cos \omega_{2}(t) & \ldots \\
\vdots & \vdots & \vdots & \vdots & \ddots
\end{array}\right)
$$

For $t \in \sigma_{1}^{( \pm)} \cap \Delta$ we put

$$
\widetilde{\mathcal{J}}(t)=\left(\begin{array}{cccccc}
1 & 0 & 0 & \ldots & 0 & 0 \\
0 & \cos \omega_{1}(t) & \sin \omega_{1}(t) & \ldots & 0 & 0 \\
0 & \sin \omega_{1}(t) & -\cos \omega_{1}(t) & \ldots & 0 & 0 \\
\vdots & \vdots & \vdots & \ddots & \vdots & \vdots \\
0 & 0 & 0 & \ldots & \cos \omega_{k}(t) & \sin \omega_{k}(t) \\
0 & 0 & 0 & \ldots & \sin \omega_{k}(t) & -\cos \omega_{k}(t)
\end{array}\right)
$$

while for $t \in \sigma_{1}^{( \pm)} \cap \delta_{\varrho}$ we put

$$
\widetilde{\mathcal{J}}(t)=\left(\begin{array}{ccccc}
\cos \omega_{1}(t) & \sin \omega_{1}(t) & \ldots & 0 & 0 \\
\sin \omega_{1}(t) & -\cos \omega_{1}(t) & \ldots & 0 & 0 \\
\vdots & \vdots & \ddots & \vdots & \vdots \\
0 & 0 & \ldots & \cos \omega_{k}(t) & \sin \omega_{k}(t) \\
0 & 0 & \ldots & \sin \omega_{k}(t) & -\cos \omega_{k}(t)
\end{array}\right) .
$$

$\left(\tilde{\mathcal{J}}(t)\right.$ is defined on $\widetilde{E}(t)=\operatorname{span}\left\{e_{k}: 2 \leqslant k<\nu_{W}(t)+1\right\}$ which has even dimension for $t \in$ $\sigma_{1}^{( \pm)} \cap \delta_{\varrho}$; note that in this case we simply delete the first column and the first row from 
the matrix defined for the previous case $t \in \sigma_{1}^{( \pm)} \cap \Delta$.) If $t \in \sigma_{0} \cap \Delta$ and $\operatorname{dim} E(t)<\infty$, we put

$$
\tilde{\mathcal{J}}(t)=\left(\begin{array}{ccccccc}
1 & 0 & 0 & \ldots & 0 & 0 & 0 \\
0 & \cos \omega_{1}(t) & \sin \omega_{1}(t) & \ldots & 0 & 0 & 0 \\
0 & \sin \omega_{1}(t) & -\cos \omega_{1}(t) & \ldots & 0 & 0 & 0 \\
\vdots & \vdots & \vdots & \ddots & \vdots & \vdots & \vdots \\
0 & 0 & 0 & \ldots & \cos \omega_{k}(t) & \sin \omega_{k}(t) & 0 \\
0 & 0 & 0 & \ldots & \sin \omega_{k}(t) & -\cos \omega_{k}(t) & 0 \\
0 & 0 & 0 & \ldots & 0 & 0 & 1
\end{array}\right)
$$

(note that

$$
\widetilde{\mathcal{J}}(t)=\left(\begin{array}{ll}
1 & 0 \\
0 & 1
\end{array}\right)
$$

if $\operatorname{dim} E(t)=2)$. In the case $t \in \sigma_{0} \cap \Delta$ and $\operatorname{dim} E(t)=\infty$, we define $\widetilde{\mathcal{J}}(t)$ as

$$
\widetilde{\mathcal{J}}(t)=\left(\begin{array}{ccccccc}
1 & 0 & 0 & \ldots & 0 & 0 & \ldots \\
0 & \cos \omega_{1}(t) & \sin \omega_{1}(t) & \ldots & 0 & 0 & \ldots \\
0 & \sin \omega_{1}(t) & -\cos \omega_{1}(t) & \ldots & 0 & 0 & \ldots \\
\vdots & \vdots & \vdots & \ddots & \vdots & \vdots & \ddots \\
0 & 0 & 0 & \ldots & \cos \omega_{k}(t) & \sin \omega_{k}(t) & \ldots \\
0 & 0 & 0 & \ldots & \sin \omega_{k}(t) & -\cos \omega_{k}(t) & \ldots \\
\vdots & \vdots & \vdots & \ddots & \vdots & \vdots & \ddots
\end{array}\right) .
$$

Finally, in the case $t \in \sigma_{0} \cap \delta_{\varrho}$ in the definition of $\tilde{\mathcal{J}}$ we delete the first row and the first column from the matrix (5.4) if $\operatorname{dim} E(t)<\infty$ and we delete the first column and the first row from the matrix (5.5) if $\operatorname{dim} E(t)=\infty$.

We impose the following assumptions on the functions $\omega_{j}$ :

$\left(1^{\prime}\right)$ the operator-valued function $\tilde{\mathcal{J}}$ takes at most a countable set of values $\{\widetilde{\mathcal{J}}\}_{n \geqslant 1}$ (the order of the values is insignificant);

$\left(2^{\prime}\right)$ if $\tilde{\mathcal{J}}$ contains an entry $\cos \omega_{j}(t)$, then $\omega_{j}(t)<1 / 3 n$;

$\left(3^{\prime}\right) \omega_{j}(t) \neq 0$;

$\left(4^{\prime}\right) \omega_{j}(t) \rightarrow 0$ as $j \rightarrow \infty$.

If in the definition of $\widetilde{\mathcal{J}}_{n}$ we replace $\omega_{j}(t)$ by zero, we obtain a diagonal operator $D_{n}$ whose entries are equal to 1 or -1 . It follows from $\left(4^{\prime}\right)$ that $D_{n}-\widetilde{\mathcal{J}}_{n}$ is compact. Note that it follows from $\left(2^{\prime}\right)$ that

$$
\left\|\widetilde{\mathcal{J}}_{n}-D_{n}\right\| \leqslant 1 / n
$$

We can now define $\Lambda$ by $\Lambda=\mathcal{J}|\Lambda|$. The operators $A$ and $Q$ are defined by (3.1) and (3.3). This completes the construction. Now to prove Theorem 1.1 we have to choose a 
vector $q_{0}$ satisfying the above conditions $(1)-(3)$ and functions $\omega_{j}$ satisfying the above conditions $\left(1^{\prime}\right)-\left(4^{\prime}\right)$ so that the operator $A^{*}$ be asymptotically stable.

\section{III.6. The point spectrum of $A^{*}$ on the unit circle}

The aim of this section is to prove the following result.

LEMMA 6.1. For any choice of $q_{0}$ and $\omega_{j}$ satisfying the conditions (1)-(3) and $\left(1^{\prime}\right)-\left(4^{\prime}\right)$ of $\S \mathrm{III} .5$, the operator $A^{*}$ has no eigenvalues on $\mathbf{T}$.

Proof. The proof is based on the following trivial observation. Let $a_{11}, a_{12}, a_{21}, a_{22}$, $d_{1}, d_{2}$ be complex numbers such that $a_{12} \neq 0$ and

$$
\left(\begin{array}{c}
0 \\
d_{2}
\end{array}\right)=\left(\begin{array}{ll}
a_{11} & a_{12} \\
a_{21} & a_{22}
\end{array}\right)\left(\begin{array}{c}
0 \\
d_{1}
\end{array}\right)
$$

Then $d_{1}=d_{2}=0$.

By Lemma 4.1, $A^{*} h=\zeta h,|\zeta|=1$, if and only if $h \in \mathcal{K}_{0}^{\frac{1}{0}}, \boldsymbol{J} h \in \mathcal{K}_{0}^{+}$and $\mathcal{J} \boldsymbol{J} h=\zeta h$. Put $g=\boldsymbol{J} h=\zeta \mathcal{J} h$. Consider the representations of $h$ and $g$ in the von Neumann integral (5.1):

$$
h(t)=\sum_{k \geqslant 1} h_{k}(t) e_{k}, \quad g(t)=\sum_{k \geqslant 1} g_{k}(t) e_{k},
$$

where $h_{k}$ and $g_{k}$ are scalar functions. Since $h \perp \mathcal{K}_{0}$ and $g=\boldsymbol{J} h \perp \mathcal{K}_{0}$, it follows that $h_{1}(t)=0$ and $g_{1}(t)=0$ for all $t$. Let us show that $h_{k}$ and $g_{k}$ are identically zero for $k \geqslant 2$. Suppose that we have already proved that for $1 \leqslant k \leqslant n$.

Note that since $h_{1}(t)=g_{1}(t)=0$, the representations of $h$ and $g$ in the von Neumann integral (5.2) coincide with the representations (6.1).

If $n$ is even and $t \in \sigma_{2}^{( \pm)}$, the equality $g=J h$ implies

$$
\left(\begin{array}{c}
0 \\
g_{n+1}(t)
\end{array}\right)=\left(\begin{array}{ll}
0 & 1 \\
1 & 0
\end{array}\right)\left(\begin{array}{c}
0 \\
h_{n+1}(t)
\end{array}\right)
$$

If $n$ is even and $t \notin \sigma_{2}^{( \pm)}$, the equality $q=\zeta \mathcal{J} h$ implies

$$
\left(\begin{array}{c}
0 \\
g_{n+1}(t)
\end{array}\right)=\left(\begin{array}{cc}
\cos \omega_{j}(t) & \sin \omega_{j}(t) \\
\sin \omega_{j}(t) & -\cos \omega_{j}(t)
\end{array}\right)\left(\begin{array}{c}
0 \\
h_{n+1}(t)
\end{array}\right)
$$

In view of the above observation, in both cases we obtain $g_{n+1}(t)=h_{n+1}(t)=0$ (recall that $\sin \omega_{j}(t) \neq 0$ ).

If $n$ is odd and $t \in \sigma_{2}^{( \pm)}$, then (6.3) holds, and if $n$ is odd and $t \notin \sigma_{2}^{( \pm)}$, then (6.2) holds which again proves that $g_{n+1}(t)=h_{n+1}(t)=0$. 


\section{III.7. How to cope with $Q$}

In this section we reduce the estimation of the Fredholm spectrum of $A^{*}$ on the unit circle to that of $\mathcal{J J}$. This would be very easy if we could prove that $I-Q$ is compact. Indeed in this case $A^{*}-\mathcal{J J}=Q^{*} \mathcal{J} Q \boldsymbol{J}-\mathcal{J} J$ is compact and so if $\lambda \in \sigma_{F}\left(A^{*}\right)$, then $\lambda \epsilon$ $\sigma_{F}(\mathcal{J J})$. It is easy to see that $I-Q$ is compact if $W$ is invertible. Indeed in this case $Q=\left(W^{1 / 2}-|\Lambda|^{1 / 2}\right) W^{-1 / 2}$ and since $W^{2}-|\Lambda|^{2}$ is rank one, it follows that $W^{1 / 2}-|\Lambda|^{1 / 2}$ is compact. We shall see in $\S I I I .9$ that $I-Q$ is compact (and even Hilbert-Schmidt) if $q \in$ Range $R$ (which corresponds to the case when $\Gamma$ has a non-trivial kernel). However we do not know whether it is possible to choose $q \notin$ Range $R$ so that $I-Q$ be compact. We also do not know whether it is possible to find $q$ so that $I-Q$ be non-compact. Note that $I-Q$ is not a Hilbert-Schmidt operator in general.

Nevertheless the following lemmas will allow us to get rid of $Q$ even in the case $\operatorname{Ker} \Gamma=\{\mathbf{0}\}$.

LEMMA 7.1. There exists a function $\varkappa \in L^{\infty}(\lambda)$ such that $\varkappa(t)>0, \lambda$-a.e., and the operator $M_{\psi} U_{0}(I-Q)$ is a compact operator from $L^{2}(\varrho)$ to $L^{2}(\lambda)$ for any $\psi$ satisfying $|\psi| \leqslant \varkappa$, where $M_{\psi}$ is multiplication by $\psi$ on $L^{2}(\lambda)$.

As before we identify $L^{2}(\varrho)$ with $\mathcal{K}_{0}$. The proof of Lemma 7.1 will be given in §III.9.

LEMMA 7.2. Suppose that the function $\omega_{1}$ in the definition of $\mathcal{J}$ satisfies the inequality $\left|\sin \omega_{1}(t)\right| \leqslant \varkappa(t)$ for $t \in \sigma_{2}^{(+)} \cup \sigma_{2}^{(-)}$, where $\varkappa$ is the function from Lemma 7.1. Then $\sigma_{F}\left(A^{*}\right) \cap \mathbf{T} \subset \sigma_{F}(\mathcal{J J})$.

Proof. Let us verify the hypothesis of Lemma 4.2. Let

$$
\mathcal{K}_{j}=\left\{f \in \mathcal{K}: f(t)=\varphi(t) e_{j+1}, \varphi \in L^{2}(\varrho)\right\}, \quad j \geqslant 0 .
$$

Clearly $\mathcal{K}_{j}$ can naturally be identified with a subspace of $L^{2}(\varrho)$. Then $\mathcal{K}=\bigoplus_{j \geqslant 0} \mathcal{K}_{j}$. Let $\left\{\mathcal{J}_{j k}\right\}_{j, k \geqslant 0}$ be the block-matrix representation of the operator $\mathcal{J}=U^{*} \tilde{\mathcal{J}} U$ with respect to the orthogonal decomposition $\mathcal{K}=\bigoplus_{j \geqslant 0} \mathcal{K}_{j}$. It follows from the definition of $\mathcal{J}$ that

$$
\mathcal{J}_{00}=U_{0}^{*}\left(I-M_{u}\right) U_{0}, \quad \mathcal{J}_{01}=U_{0}^{*} M_{v} \mid \mathcal{K}_{1}, \quad \mathcal{J}_{10}=M_{v} U_{0},
$$

where $M_{u}$ and $M_{v}$ are multiplications on $L^{2}(\lambda)$ by the functions $u$ and $v$ defined by

$$
\begin{aligned}
& u(t)= \begin{cases}1-\cos \omega_{1}(t), & t \in \sigma_{2}^{(+)} \cup \sigma_{2}^{(-)}, \\
0, & t \notin \sigma_{2}^{(+)} \cup \sigma_{2}^{(-)},\end{cases} \\
& v(t)= \begin{cases}\sin \omega_{1}(t), & t \in \sigma_{2}^{(+)} \cup \sigma_{2}^{(-)}, \\
0, & t \notin \sigma_{2}^{(+)} \cup \sigma_{2}^{(-)} .\end{cases}
\end{aligned}
$$


(Note that in the case $\nu_{W}(t)=1$ we have $v(t)=0$ and so Range $\mathcal{J}_{10} \subset \mathcal{K}_{1}$.)

Since $|v(t)| \leqslant \varkappa(t)$, it is easy to see that $|u(t)| \leqslant \varkappa(t)$.

Consider now the block-matrix representation of the operator $Q^{*} \mathcal{J} Q$ with respect to the same decomposition of $\mathcal{K}$. It is easy to see that

$$
Q^{*} \mathcal{J} Q=\left(\begin{array}{cccc}
Q_{0}^{*} U_{0}^{*}\left(I-M_{u}\right) U_{0} Q_{0} & Q_{0}^{*} U_{0}^{*} M_{v} \mid \mathcal{K}_{1} & \mathcal{J}_{02} & \ldots \\
M_{v} U_{0} Q_{0} & \mathcal{J}_{11} & \mathcal{J}_{12} & \ldots \\
\mathcal{J}_{20} & \mathcal{J}_{21} & \mathcal{J}_{22} & \ldots \\
\vdots & \vdots & \vdots & \ddots
\end{array}\right)
$$

To verify the hypotheses of Lemma 4.2 it is sufficient to prove that for any $g_{n}, h_{n} \in \mathcal{K}_{0}$ such that $\left\|g_{n}\right\|=\left\|h_{n}\right\|=1, \lim _{n \rightarrow \infty}\left\|Q_{0} g_{n}\right\|=1$ and $\mathrm{w}-\lim g_{n}=\mathrm{w}-\lim h_{n}=0$ the following equalities hold:

$$
\begin{gathered}
\lim _{n \rightarrow \infty}\left\|\left(Q_{0}^{*} U_{0}^{*}\left(I-M_{u}\right) U_{0} Q_{0}-U_{0}^{*}\left(I-M_{u}\right) U_{0}\right) g_{n}\right\|=0, \\
\lim _{n \rightarrow \infty}\left\|\left(M_{v} U_{0} Q_{0}-M_{v} U_{0}\right) g_{n}\right\|=0, \\
\lim _{n \rightarrow \infty}\left\|\left(Q_{0}^{*} U_{0}^{*} M_{v}-U_{0}^{*} M_{v}\right) h_{n}\right\|=0 .
\end{gathered}
$$

Since $|v(t)| \leqslant \varkappa(t)$, it follows from Lemma 7.1 that the operator $M_{v} U_{0} Q_{0}-M_{v} U_{0}=$ $M_{v} U_{0}\left(Q_{0}-I\right)$ is compact which implies (7.2). Similarly $Q_{0}^{*} U_{0}^{*} M_{v}-U_{0}^{*} M_{v}$ is compact and so (7.3) holds. Let us prove (7.1). We have

$$
\begin{aligned}
& Q_{0}^{*} U_{0}^{*}\left(I-M_{u}\right) U_{0} Q_{0}-U_{0}^{*}\left(I-M_{u}\right) U_{0} \\
& \quad=Q_{0}^{*} Q_{0}-I-\left(Q_{0}^{*}-I\right) U_{0}^{*} M_{u} U_{0}-U_{0}^{*} M_{u} U_{0}\left(Q_{0}-I\right)-\left(Q_{0}^{*}-I\right) U_{0}^{*} M_{u} U_{0}\left(Q_{0}-I\right) .
\end{aligned}
$$

By Lemma 7.1 the operators

$$
\left(Q_{0}^{*}-I\right) U_{0}^{*} M_{u} U_{0}, \quad U_{0}^{*} M_{u} U_{0}\left(Q_{0}-I\right), \quad \text { and } \quad\left(Q_{0}^{*}-I\right) U_{0}^{*} M_{u} U_{0}\left(Q_{0}-I\right)
$$

are compact. Hence it remains to show that $\lim _{n \rightarrow \infty}\left\|Q_{0}^{*} Q_{0} g_{n}-g_{n}\right\|=0$. Since $Q_{0}$ is a contraction and $\lim _{n \rightarrow \infty}\left(Q_{0}^{*} Q_{0} g_{n}, g_{n}\right)=\lim _{n \rightarrow \infty}\left\|Q_{0} g_{n}\right\|^{2}=1$, we have

$$
\lim _{n \rightarrow \infty}\left\|Q_{0}^{*} Q_{0} g_{n}\right\|=1
$$

It follows now from the spectral theorem for the self-adjoint contraction $Q_{0}^{*} Q_{0}$ that $\lim _{n \rightarrow \infty}\left\|Q_{0}^{*} Q_{0} g_{n}-g_{n}\right\|=0$ which proves (7.1) and completes the proof of the lemma. 


\section{III.8. The Fredholm spectrum of $\mathcal{J} J$}

As we have already mentioned, to prove the main result it is sufficient to show that we can choose the functions $q_{0}$ and $\omega_{j}$ so that $\sigma_{F}(\mathcal{J} J)$ be at most countable.

Let us briefly explain the idea how to make $\sigma_{F}(\mathcal{J J})$ at most countable. Suppose for a while that $\varrho=\lambda$ and $\mathcal{J}$ is multiplication by $\widetilde{\mathcal{J}}$ on $\mathcal{K}$. Since both functions $J$ and $\widetilde{\mathcal{J}}$ take at most countably many values, so does $J \tilde{\mathcal{J}}$. It can easily be shown that if the functions $\omega_{j}$ satisfy the conditions $\left(1^{\prime}\right)-\left(4^{\prime}\right)$ of $\S I I I .5$, then for each $t$ the operator $J(t) \tilde{\mathcal{J}}(t)$ is a small perturbation of a block-diagonal operator whose diagonal blocks can be the form

$$
\pm 1, \quad \pm\left(\begin{array}{ll}
0 & 1 \\
1 & 0
\end{array}\right)
$$

The spectrum of each such block is contained in the set $\{1,-1, i,-i\}$ and it is easy to see from (5.6) that the set $\bigcup_{t} \sigma(J(t) \widetilde{\mathcal{J}}(t))$ is at most countable and can have accumulation points only in the set $\{1,-1, i,-i\}$. Since the operator $J(t) \tilde{\mathcal{J}}(t)$ is unitary for each $t$, it follows that

$$
\sigma(\mathcal{J J}) \subset \operatorname{clos}\left(\bigcup_{t} \sigma(J(t) \tilde{\mathcal{J}}(t))\right)
$$

and so $\sigma(\mathcal{J} J)$ is at most countable.

However the operators $J$ and $\mathcal{J}$ are multiplications by $J$ and $\tilde{\mathcal{J}}$ in different systems of coordinates. Nevertheless if these two systems of coordinates are "sufficiently close" to each other, we can use similar considerations to prove that $\sigma(\mathcal{J J})$ is at most countable.

THEOREM 8.1. There exist a function $q_{0}$ in $L^{2}(\varrho)$ that satisfies the conditions

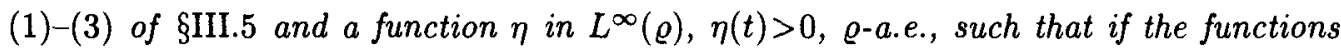
$\omega_{j}$ satisfy the conditions $\left(1^{\prime}\right)-\left(4^{\prime}\right)$ of §III.5 and $\left|\omega_{1}(t)\right| \leqslant \eta(t)$ for almost all $t \in \sigma_{2}^{(+)} \cup \sigma_{2}^{(-)}$, then $\sigma_{F}(\mathcal{J J})$ is at most countable.

To prove the theorem we represent the operator $\mathcal{J}$ as a product of 3 operators. We define the operator-valued functions $\widetilde{\mathcal{J}}_{2}^{(+)}, \widetilde{\mathcal{J}}_{2}^{(-)}$, and $\widetilde{\mathcal{J}}_{1}$ on $\Delta \cup \delta_{\lambda} \cup \delta_{\varrho}$ by

$$
\begin{aligned}
\widetilde{\mathcal{J}}_{2}^{( \pm)}(t) & = \begin{cases}\tilde{\mathcal{J}}(t), & t \in \sigma_{2}^{( \pm)}, \\
I, & t \notin \sigma_{2}^{( \pm)}\end{cases} \\
\widetilde{\mathcal{J}}_{1}(t) & = \begin{cases}\widetilde{\mathcal{J}}(t), & t \notin \sigma_{2}^{(+)} \cup \sigma_{2}^{(-)} \\
I, & t \in \sigma_{2}^{(+)} \cup \sigma_{2}^{(-)}\end{cases}
\end{aligned}
$$

Let $\tilde{\mathcal{J}}_{2}^{(+)}, \tilde{\mathcal{J}}_{2}^{(-)}$, and $\widetilde{\mathcal{J}}_{1}$ be multiplications by $\widetilde{\mathcal{J}}_{2}^{(+)}, \widetilde{\mathcal{J}}_{2}^{(-)}$, and $\tilde{\mathcal{J}}_{1}$ on the von Neumann integral (5.2). Put $\mathcal{J}_{2}^{( \pm)}=U^{*} \widetilde{\mathcal{J}}_{2}^{( \pm)} U$ and $\mathcal{J}_{1}=U^{*} \widetilde{\mathcal{J}}_{1} U$. Clearly $\mathcal{J}=\mathcal{J}_{2}^{(+)} \mathcal{J}_{2}^{(-)} \mathcal{J}_{1}$. It is easy to see that $\mathcal{K}_{0}$ is a reducing subspace for $\mathcal{J}_{1}$ and $\mathcal{J}_{1} \mid \mathcal{K}_{0}=I$. 
For a Borel subset $\gamma$ of $\sigma(W)$ we denote by $\mathcal{K}(\gamma)$ the subspace of $\mathcal{K}$ that consists of functions supported on $\gamma$ and by $\mathcal{E}(\gamma)$ the orthogonal projection onto $\mathcal{K}(\gamma)$ (in other words $\mathcal{E}$ is the spectral measure of $W)$. Put $\sigma_{1}=\sigma(W) \backslash\left(\sigma_{2}^{(+)} \cup \sigma_{2}^{(-)}\right)$.

We shall call an operator on $\mathcal{K}$ almost block diagonal (with respect to the decomposition $\left.\mathcal{K}=\mathcal{K}\left(\sigma_{1}\right) \oplus \mathcal{K}\left(\sigma_{2}^{(+)}\right) \oplus \mathcal{K}\left(\sigma_{2}^{(-)}\right)\right)$if its block matrix has compact off diagonal entries.

It is easy to see from the definition of $\mathcal{J}$ that the operator $\mathcal{J}_{1}$ is block diagonal. It is also easy to show that the operators $\mathcal{J}_{2}^{(+)}$and $\mathcal{J}_{2}^{(-)}$are almost block diagonal if the operators

$$
\left(\mathcal{J}_{2}^{( \pm)}-I\right) \mathcal{E}\left(\sigma(W) \backslash \sigma_{2}^{( \pm)}\right)
$$

are compact. Therefore in this case $\mathcal{J}$ is also almost block diagonal. It follows easily from the compactness of the operators (8.1) that the operators

$$
\mathcal{E}\left(\sigma_{2}^{( \pm)}\right) \mathcal{J} \mathcal{E}\left(\sigma_{2}^{( \pm)}\right)-\mathcal{E}\left(\sigma_{2}^{( \pm)}\right) \mathcal{J}_{2}^{( \pm)} \mathcal{E}\left(\sigma_{2}^{( \pm}\right), \quad \mathcal{E}\left(\sigma_{1}\right) \mathcal{J} \mathcal{E}\left(\sigma_{1}\right)-\mathcal{E}\left(\sigma_{1}\right) \mathcal{J}_{1} \mathcal{E}\left(\sigma_{1}\right)
$$

are also compact.

The following lemma proves the compactness of the operators (8.1).

LEMMA 8.2. There exists a function $q_{0}$ in $L^{2}(\varrho)$ that satisfies the conditions (1)-(3) of §III.5 and a function $\eta$ in $L^{\infty}(\varrho), \eta(t)>0, \varrho$-a.e., such that if the functions $\omega_{j}$ satisfy the conditions $\left(1^{\prime}\right)-\left(4^{\prime}\right)$ of $\S I I I .5$ and $\left|\omega_{1}(t)\right| \leqslant \eta(t)$ for almost all $t \in \sigma_{2}^{(+)} \cup \sigma_{2}^{(-)}$, then the operators (8.1) are compact.

The proof of Lemma 8.2 will be given in $\S I I I .9$.

Proof of Theorem 8.1. Let us choose $q_{0}, \eta$, and $\omega_{j}$ satisfying the hypotheses of Lemma 8.2. Then the operator $\mathcal{J}$ is almost block diagonal with respect to the decomposition $\mathcal{K}=\mathcal{K}\left(\sigma_{1}\right) \oplus \mathcal{K}\left(\sigma_{2}^{(+)}\right) \oplus \mathcal{K}\left(\sigma_{2}^{(-)}\right)$. Obviously the operator $\boldsymbol{J}$ is block diagonal. Hence $\mathcal{J J}$ is almost block diagonal. Therefore to prove that $\sigma_{F}(\mathcal{J J})$ is at most countable, it is sufficient to prove that the Fredholm spectrum of each diagonal block is at most countable on the unit circle.

Consider the block

$$
\mathcal{E}\left(\sigma_{1}\right) \mathcal{J} \boldsymbol{J}\left|\mathcal{K}\left(\sigma_{1}\right)=\mathcal{E}\left(\sigma_{1}\right) \mathcal{J} \mathcal{E}\left(\sigma_{1}\right) \boldsymbol{J}\right| \mathcal{K}\left(\sigma_{1}\right) .
$$

It follows from the compactness of the operators $(8.2)$ that $\sigma_{F}\left(\mathcal{E}\left(\sigma_{1}\right) \mathcal{J} J \mid \mathcal{K}\left(\sigma_{1}\right)\right)=$ $\sigma_{F}\left(\mathcal{E}\left(\sigma_{1}\right) \mathcal{J}_{1} \boldsymbol{J} \mid \mathcal{K}\left(\sigma_{1}\right)\right)$. But both operators $\mathcal{E}\left(\sigma_{1}\right) \mathcal{J}_{1} \mathcal{E}\left(\sigma_{1}\right)$ and $\mathcal{E}\left(\sigma_{1}\right) \boldsymbol{J} \mathcal{E}\left(\sigma_{1}\right)$ are multiplications on the von Neumann integral (5.1). So the reasoning in the beginning of this section works in this case and hence $\sigma\left(\mathcal{E}\left(\sigma_{1}\right) \mathcal{J} J \mid \mathcal{K}\left(\sigma_{1}\right)\right)$ is at most countable.

The situation with two other blocks is a bit more complicated. Consider for example the block

$$
\mathcal{E}\left(\sigma_{2}^{(+)}\right) \mathcal{J} \boldsymbol{J}\left|\mathcal{K}\left(\sigma_{2}^{(+)}\right)=\mathcal{E}\left(\sigma_{2}^{(+)}\right) \mathcal{J E}\left(\sigma_{2}^{(+)}\right) \boldsymbol{J}\right| \mathcal{K}\left(\sigma_{2}^{(+)}\right)
$$


Again by the compactness of the operators (8.2) we have

$$
\sigma_{F}\left(\mathcal{E}\left(\sigma_{2}^{(+)}\right) \mathcal{J} \boldsymbol{J} \mid \mathcal{K}\left(\sigma_{2}\right)\right)=\sigma_{F}\left(\mathcal{E}\left(\sigma_{2}^{(+)}\right) \mathcal{J}_{2} \boldsymbol{J} \mid \mathcal{K}\left(\sigma_{2}\right)\right)
$$

Let $J_{2}^{(+)}$be the operator-valued function defined by

$$
J_{2}^{(+)}(t)= \begin{cases}J(t), & t \in \sigma_{2}^{(+)} \\ I, & t \in \sigma(W) \backslash \sigma_{2}^{(+)}\end{cases}
$$

and let $\boldsymbol{J}_{2}^{(+)}$be multiplication by $J_{2}^{(+)}$on the von Neumann integral (5.1). Obviously $\mathcal{E}\left(\sigma_{2}^{(+)}\right) \boldsymbol{J} \mathcal{E}\left(\sigma_{2}^{(+)}\right)=\mathcal{E}\left(\sigma_{2}^{(+)}\right) \boldsymbol{J}_{2}^{(+)} \mathcal{E}\left(\sigma_{2}^{(+)}\right)$and so

$$
\begin{aligned}
\mathcal{E}\left(\sigma_{2}^{(+)}\right) \mathcal{J}_{2}^{(+)} \mathcal{J E}\left(\sigma_{2}^{(+)}\right) & =\mathcal{E}\left(\sigma_{2}^{(+)}\right) \mathcal{J}_{2}^{(+)} \mathcal{E}\left(\sigma_{2}^{(+)}\right) \mathcal{J} \mathcal{E}\left(\sigma_{2}^{(+)}\right) \\
& =\mathcal{E}\left(\sigma_{2}^{(+)}\right) \mathcal{J}_{2}^{(+)} \mathcal{E}\left(\sigma_{2}^{(+)}\right) \boldsymbol{J}_{2}^{(+)} \mathcal{E}\left(\sigma_{2}^{(+)}\right) \\
& =\mathcal{E}\left(\sigma_{2}^{(+)}\right) \mathcal{J}_{2}^{(+)} \boldsymbol{J}_{2}^{(+)} \mathcal{E}\left(\sigma_{2}^{(+)}\right)
\end{aligned}
$$

We have

$$
\mathcal{J}_{2}^{(+)} J_{2}^{(+)}=U^{*} \widetilde{\mathcal{J}}_{2}^{(+)} U J_{2}^{(+)}=U^{*} \tilde{\mathcal{J}}_{2}^{(+)}\left(U \boldsymbol{J}_{2}^{(+)} U^{*}\right) U
$$

Since $\mathcal{K}_{0}$ is a reducing subspace of $J_{2}^{(+)}$, it follows from (5.3) that the operator $\tilde{J}_{2}^{(+)}=U \boldsymbol{J}_{2}^{(+)} U^{*}$ is multiplication on the von Neumann integral $(5.2)$ by the operatorvalued function $\tilde{J}_{2}^{(+)}$defined by

$$
\tilde{J}_{2}^{(+)}(t) e_{j}= \begin{cases}J_{2}^{(+)}(t) e_{j}, & 2 \leqslant j<\nu_{W}+1, t \in \delta_{\varrho} \cup \Delta \\ e_{1}, & j=1, t \in \delta_{\varrho} \cup \Delta \cup \delta_{\lambda}\end{cases}
$$

Now the operators $\tilde{\mathcal{J}}_{2}^{(+)}$and $U J_{2}^{(+)} U^{*}$ are both multiplications on (5.2) and so the reasoning in the beginning of this section works which shows that $\sigma\left(\tilde{\mathcal{J}}_{2}^{(+)} U J_{2}^{(+)} U^{*}\right)=$ $\sigma\left(\mathcal{J}_{2}^{(+)} \boldsymbol{J}^{\prime}\right)$ is at most countable.

To show that $\sigma\left(\mathcal{E}\left(\sigma_{2}^{(+)}\right) \mathcal{J}_{2}^{(+)} J_{2}^{(+)} \mid \mathcal{K}\left(\sigma_{2}^{(+)}\right)\right) \cap \mathbf{T}$ is at most countable we need the following lemma.

LEMMA 8.3. Let $Z$ be a contraction and $V$ a unitary operator on Hilbert space. Then

$$
\sigma\left(Z^{*} V Z\right) \cap \mathbf{T} \subset \sigma(V)
$$

Let us first complete the proof of Theorem 8.1. It follows from Lemma 8.3 that

$$
\sigma\left(\mathcal{E}\left(\sigma_{2}^{(+)}\right) \mathcal{J}_{2}^{(+)} \boldsymbol{J}_{2}^{(+)} \mathcal{E}\left(\sigma_{2}^{(+)}\right)\right) \cap \mathbf{T} \subset \sigma\left(\mathcal{J}_{2}^{(+)} \boldsymbol{J}_{2}^{(+)}\right)
$$


which implies that $\sigma\left(\mathcal{E}\left(\sigma_{2}^{(+)}\right) \mathcal{J}_{2}^{(+)} \boldsymbol{J}_{2}^{(+)} \mid \mathcal{K}\left(\sigma_{2}^{(+)}\right)\right) \cap \mathbf{T}$ is at most countable. In a similar way it can be shown that $\sigma\left(\mathcal{E}\left(\sigma_{2}^{-)}\right) \mathcal{J} J \mathcal{E}\left(\sigma_{2}^{(-)}\right)\right) \cap \mathbf{T}$ is at most countable which completes the proof.

Proof of Lemma 8.3. Suppose that $\zeta \in \sigma\left(Z^{*} V Z\right) \cap \mathbf{T}$ and $\zeta \notin \sigma(V)$. Since $Z^{*} V Z$ is a contraction and $|\zeta|=1$, it follows that there exists a sequence of vectors $\left\{f_{n}\right\}_{n \geqslant 0}$ such that $\left\|f_{n}\right\|=1$ and

$$
\left(\left(\bar{\zeta} Z^{*} V Z-I\right) f_{n}, f_{n}\right)=\bar{\zeta}\left(Z^{*} V Z f_{n}, f_{n}\right)-\left\|f_{n}\right\|^{2} \rightarrow 0 .
$$

Since $\zeta \notin \sigma(V)$, we have $1 \notin \sigma(\bar{\zeta} V)$ and it follows from the spectral theorem for unitary operators that there exists a positive $\varepsilon$ such that

$$
\operatorname{Re}(\bar{\zeta} V g, g) \leqslant(1-\varepsilon)\|g\|^{2}
$$

for any $g$. Hence

$$
\operatorname{Re}\left(\left(\bar{\zeta} Z^{*} V Z-I\right) f_{n}, f_{n}\right)=\operatorname{Re} \bar{\zeta}\left(Z^{*} V Z f_{n}, f_{n}\right)-\left\|f_{n}\right\| \leqslant(1-\varepsilon)\left\|Z f_{n}\right\|^{2}-1 \leqslant-\varepsilon
$$

which contradicts (8.3).

Now we are in a position to prove Theorem 1.1.

Proof of Theorem 1.1. We can choose the functions $q_{0}$ and $\omega_{j}$ that satisfy the hypotheses of Theorem 8.1 and Lemma 7.2. It follows from Lemma 6.1 that $A^{*}$ has no eigenvalues on $\mathbf{T}$. Next it follows from Lemma 7.2 that $\sigma_{F}\left(A^{*}\right) \cap \mathbf{T} \subset \sigma_{F}(\mathcal{J J})$ and so by Theorem 8.1 the set $\sigma_{F}\left(A^{*}\right)$ is at most countable. Consequently, by the stability test (see §III.4) the operator $A^{*}$ is asymptotically stable which by Lemmas 3.1 and $3.2 \mathrm{imply}$ that $T$ is asymptotically stable. The result now follows from Theorem 2.1 .

\section{III.9. Compact integral operators}

In this section we prove Lemmas 7.1 and 8.2. The following lemma will help us to represent the operator $U_{0}\left(I-Q_{0}\right)$ as an integral operator.

Recall that the space $\mathcal{K}_{0}$ is reducing for both $W^{2}$ and $\Lambda^{2}$. As usual we identify $\mathcal{K}_{0}$ with $L^{2}(\varrho)$. Clearly $M \stackrel{\text { def }}{=} W \mid \mathcal{K}_{0}$ is multiplication by the independent variable on $L^{2}(\varrho)$. Let $\widetilde{M}$ be multiplication by the independent variable on $L^{2}(\lambda)$. Then $|\Lambda| \mid \mathcal{K}_{0}=U^{*} \widetilde{M} U$ and

$$
\left(W^{2}-\Lambda^{2}\right) \mid \mathcal{K}_{0}=M^{2}-U_{0}^{*} \tilde{M}^{2} U_{0}=(\cdot, p) p
$$


LEMMA 9.1. Let $r=U_{0} p \in L^{2}(\lambda)$. Then for any continuously differentiable function $\varphi$ on $\operatorname{supp} \varrho \cup \operatorname{supp} \lambda$ the following equality holds:

$$
\left(\left(U_{0} \varphi\left(M^{2}\right)-\varphi\left(\tilde{M}^{2}\right) U_{0}\right) f\right)(t)=\int \frac{\varphi\left(t^{2}\right)-\varphi\left(s^{2}\right)}{t^{2}-s^{2}} r(t) \overline{p(s)} f(s) d \varrho(s) .
$$

Note that Lemma 9.1 is a consequence of the theory of double operator integrals (see [BS2]). We prove it here for the sake of completeness.

Proof of Lemma 9.1. It follows from (9.1) that

$$
U_{0} M^{2}-\tilde{M}^{2} U_{0}=(\cdot, p) r
$$

which is equivalent to the equality

$$
\left(\left(U M_{0}^{2}-\tilde{M}_{0}^{2} U\right) f\right)(t)=\int r(t) \overline{p(s)} f(s) d \varrho(s) .
$$

Clearly

$$
U_{0} M^{4}-\tilde{M}^{4} U_{0}=\left(U_{0} M^{2}-\tilde{M}^{2} U_{0}\right) M^{2}+\tilde{M}^{2}\left(U_{0} M^{2}-\tilde{M}^{2} U_{0}\right),
$$

which is equivalent to

$$
\left(\left(U_{0} M^{4}-\tilde{M}^{4} U_{0}\right) f\right)(t)=\int\left(s^{2}+t^{2}\right) r(t) \overline{p(s)} f(s) d \varrho(s) .
$$

Similarly

$$
\left(\left(U_{0} M^{2 n}-\tilde{M}^{2 n} U_{0}\right) f\right)(t)=\int \frac{t^{2 n}-s^{2 n}}{t^{2}-s^{2}} r(t) \overline{p(s)} f(s) d \varrho(s)
$$

which proves $(9.2)$ for $\varphi(t)=t^{2 n}$. The result follows now from the fact that the set of polynomials of $t^{2}$ is dense in $C^{1}\left[0,\|W\|^{2}\right]$ and the fact that the Hilbert-Schmidt norm of the integral operator on the right-hand side of (9.2) is at most const $\|\varphi\|_{C^{1}}$.

Proof of Lemma 7.1. Let $\varphi(s)=s^{1 / 4}, s>0$. It follows from Lemma 9.1 that

$$
\left(\left(U_{0} M^{1 / 2}-\tilde{M}^{1 / 2} U_{0}\right) f\right)(t)=\int \frac{t^{1 / 2}-s^{1 / 2}}{t^{2}-s^{2}} r(t) \overline{p(s)} f(s) d \varrho(s)
$$

at least in the case when $0 \notin \operatorname{supp} f$. Indeed in this case we can change $\varphi$ in a small neighborhood of 0 to make it continuously differentiable.

On the other hand,

$$
I-Q=I-|\Lambda|^{1 / 2} W^{-1 / 2}=\left(W^{1 / 2}-|\Lambda|^{1 / 2}\right) W^{-1 / 2}
$$


on the dense subset Range $W^{1 / 2}$ of $\mathcal{K}$. Therefore

$$
I-Q_{0}=\left(M^{1 / 2}-U_{0}^{*} \tilde{M}^{1 / 2} U_{0}\right) M^{-1 / 2}
$$

on the range of $M^{1 / 2}$. It follows from (9.3) that

$$
\begin{aligned}
\left(U_{0}\left(I-Q_{0}\right) f\right)(t) & =\left(\left(U_{0} M^{1 / 2}-\widetilde{M}^{1 / 2} U_{0}\right) M^{-1 / 2} f\right)(t) \\
& =\int \frac{t^{1 / 2}-s^{1 / 2}}{t^{2}-s^{2}} r(t) \overline{p(s)} s^{-1 / 2} f(s) d \varrho(s)
\end{aligned}
$$

whenever $0 \notin \operatorname{supp} f$. Since $p=W q_{0}$ we have

$$
\left(U_{0}\left(I-Q_{0}\right) f\right)(t)=\int \frac{t^{1 / 2}-s^{1 / 2}}{t^{2}-s^{2}} r(t) \overline{q_{0}(s)} s^{1 / 2} f(s) d \varrho(s)
$$

whenever $0 \notin \operatorname{supp} f$.

Consider first the case $q_{0} \notin$ Range $W$ (i.e. Ker $\Gamma=\{0\}$ ). In this case $p \notin$ Range $W^{2}$ and so $\operatorname{Ker} \Lambda=\{0\}$. Therefore $\lambda(\{0\})=0$. Consequently, for $\lambda$-almost all $t$ the function

$$
s \mapsto \frac{t^{1 / 2}-s^{1 / 2}}{t^{2}-s^{2}} s^{1 / 2} \overline{q_{0}(s)}
$$

belongs to $L^{2}(\varrho)$. Therefore there exists a function $\varkappa$ in $L^{\infty}(\lambda)$ such that $\varkappa(t)>0, \lambda$-a.e., and

$$
\iint\left|\frac{t^{1 / 2}-s^{1 / 2}}{t^{2}-s^{2}} s^{1 / 2} r(t) \overline{q_{0}(s)} \varkappa(t)\right|^{2} d \varrho(s) d \lambda(t)<\infty .
$$

It follows that the operator $M_{\varphi} U\left(I-Q_{0}\right)$ is Hilbert-Schmidt whenever $|\varphi(t)| \leqslant \varkappa(t)$, $\lambda$-a.e.

Consider now the case $q_{0} \in$ Range $W$. Let us show that in this case $\left(I-Q_{0}\right)$ is a Hilbert-Schmidt operator. Let $q_{0}=W h, h \in L^{2}(\varrho)$. It follows from (9.4) that

$$
\left(U_{0}\left(I-Q_{0}\right) f\right)(t)=\int \frac{t^{1 / 2} s^{3 / 2}-s^{2}}{t^{2}-s^{2}} \overline{h(s)} r(t) f(s) d \varrho(s)
$$

whenever $0 \in \operatorname{supp} f$. However it is easy to see that

$$
\sup _{t, s>0}\left|\frac{t^{1 / 2} s^{3 / 2}-s^{2}}{t^{2}-s^{2}}\right|<\infty
$$

and so the integral operator on the right-hand side of (9.5) is Hilbert-Schmidt.

To prove Lemma 8.2 we need one more lemma. We denote by $\mathcal{E}_{0}(\gamma)$ the orthogonal projection on $L^{2}(\varrho)$ onto the subspace of functions supported on $\gamma$ and by $\mathcal{F}_{0}(\gamma)$ the corresponding projection on $L^{2}(\lambda)$. Note that $\mathcal{E}_{0}$ is the spectral measure of $W \mid \mathcal{K}_{0}$ and $\mathcal{F}_{0}$ is the spectral measure of $U\left(|\Lambda| \mid \mathcal{K}_{0}\right) U^{*}$. As usual $M_{\psi}$ denotes multiplication by $\psi$. 
LEMMA 9.2. There exist a function $q_{0}$ satisfying the conditions (1)-(3) of §III.5 and a function $\eta$ which is positive Q-a.e. on $\sigma_{2}^{(+)} \cup \sigma_{2}^{(-)}$such that

$$
M_{\psi} \mathcal{F}_{0}\left(\sigma_{2}^{( \pm)}\right) U_{0} \mathcal{E}_{0}\left(\sigma(W) \backslash \sigma_{2}^{( \pm)}\right)
$$

are compact operators from $L^{2}(\varrho)$ to $L^{2}(\lambda)$ for any function $\psi$ such that $|\psi(t)| \leqslant \eta(t)$ a.e.

Proof. Let us first show that for any disjoint compact sets $\gamma_{1}$ and $\gamma_{2}$

$$
\left(\mathcal{F}_{0}\left(\gamma_{2}\right) U \mathcal{E}_{0}\left(\gamma_{1}\right) f\right)(t)=\int_{\gamma_{1}} \frac{f(s)}{s^{2}-t^{2}} r(t) \overline{p(s)} d \varrho(s), \quad t \in \gamma_{2}
$$

Let $\varphi$ be a continuously differentiable function such that

$$
\varphi(s)= \begin{cases}1, & s^{1 / 2} \in \gamma_{1}, \\ 0, & s^{1 / 2} \in \gamma_{2} .\end{cases}
$$

Then by Lemma 9.1

$$
\left(\left(U_{0} \varphi\left(M^{2}\right)-\varphi\left(\tilde{M}^{2}\right) U_{0}\right) f\right)(t)=\int \frac{\varphi\left(s^{2}\right)-\varphi\left(t^{2}\right)}{s^{2}-t^{2}} r(t) \overline{p(s)} d \varrho(s) .
$$

Clearly $\left(\varphi\left(\tilde{M}^{2}\right) U_{0} f\right)(t)=0$ for $t \in \gamma_{2}$. Therefore $\mathcal{F}_{0}\left(\gamma_{2}\right) \varphi\left(\tilde{M}^{2}\right) U_{0}=0$. It is also clear that if $f$ is supported on $\gamma_{1}$, then $\varphi\left(M^{2}\right) f=f$. It follows that for $f$ supported on $\gamma_{1}$

$$
\left(U_{0} f\right)(t)=\int \frac{1}{s^{2}-t^{2}} r(t) \overline{p(s)} f(s) d \varrho(s), \quad t \in \gamma_{2}
$$

which implies (9.7).

Note that if $\gamma_{1}$ and $\gamma_{2}$ are arbitrary disjoint Borel subsets, then we can approximate them by compact subsets and pass to the limit which proves that (9.7) holds for $f$ in a dense subset of $L^{2}(\varrho)$.

Therefore to prove the lemma it is sufficient to find a function $q_{0}$ which satisfies the conditions (1)-(3) of §III.5 and such that for almost all $t \in \sigma_{2}^{( \pm)}$

$$
\int_{\sigma(W) \backslash \sigma_{2}^{( \pm)}} \frac{|p(s)|^{2}}{\left|s^{2}-t^{2}\right|} d \varrho(s)<\infty
$$

(Recall that $p=W q_{0}$ and so $p(s)=s q_{0}(s)$ ) Indeed in this case it follows from (9.7) with $\gamma_{1}=\sigma_{2}^{( \pm)}$and $\gamma_{2}=\sigma(W) \backslash \sigma_{2}^{( \pm)}$that if $\eta$ is a positive function such that

$$
\int_{\sigma_{2}^{( \pm)}}|r(t)|^{2}(\eta(t))^{2}\left(\int_{\sigma(W) \backslash \sigma_{2}^{( \pm)}} \frac{|p(s)|^{2}}{\left|s^{2}-t^{2}\right|} d \varrho(s)\right) d \lambda(t)<\infty
$$


then the operators (9.6) are Hilbert-Schmidt provided $|\psi| \leqslant \eta$.

Let $\left\{\beta_{n}^{(+)}\right\}_{n \geqslant 1},\left\{\beta_{n}^{(-)}\right\}_{n \geqslant 1},\left\{\gamma_{n}\right\}_{n \geqslant 1}$ be increasing sequences of compact sets such that

$$
\beta_{n}^{( \pm)} \subset \sigma_{2}^{( \pm)}, \quad \lambda\left(\sigma_{2}^{( \pm)} \backslash \beta_{n}^{( \pm)}\right) \rightarrow 0, \quad \gamma_{n} \subset \sigma_{1}, \quad \varrho\left(\sigma_{1} \backslash \gamma_{n}\right) \rightarrow 0,
$$

where $\sigma_{1}=\sigma(W) \backslash\left(\sigma_{2}^{(+)} \cup \sigma_{2}^{(-)}\right)$. Note that since $\varrho$ and $\lambda$ are mutually absolutely continuous on $\Delta$, we have $\varrho\left(\sigma_{2}^{( \pm)} \backslash \beta_{n}^{( \pm)}\right) \rightarrow 0$.

Consider first the case $\operatorname{Ker} \Gamma \neq\{0\}$. In this case we have to choose $q_{0}$ in Range $W$ such that $\left\|q_{0}\right\|_{L^{2}(\varrho)}=1$ and $q_{0}(s) \neq 0$, $\varrho$-a.e.

We can easily define a positive function $q_{0}$ on $\beta_{1}^{(+)} \cup \beta_{1}^{(-)} \cup \gamma_{1}$ so that

$$
\begin{gathered}
\int_{\beta_{1}^{(+)} \cup \beta_{1}^{(-)} \cup \gamma_{1}} \frac{\left|q_{0}(s)\right|^{2}}{s^{2}} d \varrho(s) \leqslant 1, \\
\int_{\beta_{1}^{(-)} \cup \gamma_{1}} \frac{|p(s)|^{2}}{\left|s^{2}-t^{2}\right|^{2}} d \varrho(s) \leqslant 1, \quad t \in \beta_{1}^{(+)}, \\
\int_{\beta_{1}^{(+)} \cup \gamma_{1}} \frac{|p(s)|^{2}}{\left|s^{2}-t^{2}\right|^{2}} d \varrho(s) \leqslant 1, \quad t \in \beta_{1}^{(-)} .
\end{gathered}
$$

Then we can proceed by induction. On the $n$th step we define $q_{0}$ on

$$
\beta_{n}^{(+)} \cup \beta_{n}^{(-)} \cup \gamma_{n} \backslash\left(\beta_{n-1}^{(+)} \cup \beta_{n-1}^{(-)} \cup \gamma_{n-1}\right)
$$

so that

$$
\begin{gathered}
\int_{\beta_{n}^{(+)} \cup \beta_{n}^{(-)} \cup \gamma^{(n)}} \frac{\left|q_{0}(s)\right|^{2}}{s^{2}} d \varrho(s) \leqslant 1+\frac{1}{2}+\ldots+\frac{1}{2^{n-1}}, \\
\int_{\beta_{n}^{(-)} \cup \gamma_{n}} \frac{|p(s)|^{2}}{\left|s^{2}-t^{2}\right|^{2}} d \varrho(s) \leqslant \frac{1}{2^{k-1}}+\ldots+\frac{1}{2^{n-1}}, \quad t \in \beta_{k}^{(+)} \backslash \beta_{k-1}^{(+)}, \quad 1 \leqslant k \leqslant n, \\
\int_{\beta_{n}^{(+)} \cup \gamma_{n}} \frac{|p(s)|^{2}}{\left|s^{2}-t^{2}\right|^{2}} d \varrho(s) \leqslant \frac{1}{2^{k-1}}+\ldots+\frac{1}{2^{n-1}}, \quad t \in \beta_{k}^{(-)} \backslash \beta_{k-1}^{(-)}, \quad 1 \leqslant k \leqslant n,
\end{gathered}
$$

(here $\left.\beta_{0}^{(+)}=\beta_{0}^{(-)}=\gamma_{0}=\varnothing\right)$.

Passing to the limit, we obtain a function $q_{0}$ such that $q_{0}(s) \neq 0, \varrho$-a.e., $q_{0} \in$ Range $W$ and (9.8) holds with $p(s)=s q_{0}(s)$. The only condition which can be violated is $\left\|q_{0}\right\|_{L^{2}(\varrho)}=1$ but obviously we can multiply $q_{0}$ by a suitable constant to achieve this condition.

Consider now the case $\operatorname{Ker} \Gamma=\{0\}$. We have to construct a function $q_{0}$ such that $\left\|q_{0}\right\|_{L^{2}(\varrho)}=1, q_{0}(s) \neq 0, \varrho$-a.e., $q_{0} \notin$ Range $W$, and (9.8) holds. In this case the operator 
$W$ is non-invertible. Hence $\varrho((0, \varepsilon))>0$ for any $\varepsilon>0$. Therefore the same is true for the restriction of $\varrho$ to at least one of the sets $\sigma_{2}^{(+)}, \sigma_{2}^{(-)}, \sigma_{1}$. To be definite suppose that

$$
\varrho\left((0, \varepsilon) \cap \sigma_{2}^{(+)}\right)>0
$$

for any $\varepsilon>0$.

Clearly we can assume that $0 \in \sigma_{2}^{(+)}$. It is easy to see that there exists a compact subset $\beta_{1}^{(+)}$of $\sigma_{2}^{(+)}$such that

$$
\varrho\left((0, \varepsilon) \cap \beta_{1}^{(+)}\right)>0
$$

for any $\varepsilon>0$. We can choose now a positive function $q_{0}$ on $\beta_{1}^{(+)}$such that

$$
\int_{\beta_{1}^{(+)}} \frac{\left|q_{0}(s)\right|^{2}}{s^{2}} d \varrho(s)=\infty
$$

Then we can choose increasing sequences $\left\{\beta_{n}^{( \pm)}\right\}_{n \geqslant 1}$ and $\left\{\gamma_{n}\right\}_{n \geqslant 1}$ of compact sets satisfying (9.9) and proceed by induction as in the first case. On the $n$th step we can define $q_{0}$ on $\beta_{n}^{(+)} \cup \beta_{n}^{(-)} \cup \gamma_{n}$ so that $(9.10)$ and (9.11) hold. Passing to the limit and multiplying if necessary by a suitable constant, we obtain a function $q_{0}$ with desired properties.

Proof of Lemma 8.2. Let $p$ and $\eta$ satisfy the assumptions of Lemma 8.2. Suppose $\left|\sin \omega_{1}(t)\right| \leqslant \eta(t)$ for $t \in \sigma_{2}^{(+)} \cup \sigma_{2}^{(-)}$. It is easy to see that $1-\cos \omega_{1}(t) \leqslant \eta(t), t \in \sigma_{2}^{(+)} \cup \sigma_{2}^{(-)}$.

We have to show that the operators

$$
\left(\mathcal{J}_{2}^{( \pm)}-I\right) \mathcal{E}\left(\sigma(W) \backslash \sigma_{2}^{( \pm)}\right)
$$

are compact. Since

$$
\left(\mathcal{J}_{2}^{( \pm)}-I\right) \mathcal{E}\left(\sigma(W) \backslash \sigma_{2}^{( \pm)}\right) f=\mathbf{0}
$$

for $f \in \mathcal{K}_{0}^{\perp}$, it is sufficient to show that the operators

$$
\left(\mathcal{J}_{2}^{( \pm)}-I\right) \mathcal{E}_{0}\left(\sigma(W) \backslash \sigma_{2}^{( \pm)}\right) P_{0}
$$

are compact where $P_{0}$ is the orthonormal projection onto $\mathcal{K}_{0}$. Let us prove that the operator $\left(\mathcal{J}_{2}^{(+)}-I\right) \mathcal{E}\left(\sigma(W) \backslash \sigma_{2}^{(+)}\right) P_{0}$ is compact. The proof for the other operator is the same. It is easy to see from the definition of $\mathcal{J}_{2}^{(+)}$that for $f \in \mathcal{K}_{0}$ the vector $\left(\mathcal{J}_{2}^{(+)}-I\right) f$ can be represented in the decomposition $\mathcal{K}=\bigoplus_{j \geqslant 0} \mathcal{K}_{j}$ as

$$
\left(\mathcal{J}_{2}^{(+)}-I\right) f=\left(\begin{array}{c}
U_{0}^{*} M_{u} U_{0} f \\
M_{v} U_{0} f \\
\mathbf{0} \\
\mathbf{0} \\
\vdots
\end{array}\right)
$$


where $M_{u}$ and $M_{v}$ are multiplications on $L^{2}(\lambda)$ by the functions $u$ and $v$ defined by

$$
\begin{aligned}
& u(t)= \begin{cases}\cos \omega_{1}(t)-1, & t \in \sigma_{2}^{(+)}, \\
0, & t \notin \sigma_{2}^{(+)}\end{cases} \\
& v(t)= \begin{cases}\sin \omega_{1}(t), & t \in \sigma_{2}^{(+)} \\
0, & t \notin \sigma_{2}^{(+)}\end{cases}
\end{aligned}
$$

By Lemma 9.2 the operators $M_{u} U_{0} \mathcal{E}_{0}\left(\sigma(W) \backslash \sigma_{2}^{(+)}\right)$and $M_{v} U_{0} \mathcal{E}_{0}\left(\sigma(W) \backslash \sigma_{2}^{(+)}\right)$are compact which proves the compactness of $\left(\mathcal{J}_{2}^{(+)}-I\right) \mathcal{E}_{0}\left(\sigma(W) \backslash \sigma_{2}^{(+)}\right) P_{0}$.

\section{III.10. A theorem in perturbation theory}

In this section we find a connection between our results on Hankel operators and a theorem in perturbation theory.

In the previous sections, for an operator $\Gamma$ satisfying the conditions (C1)-(C3) we have constructed an output normal system $\{T, p, q\}$ such that the Hankel operator associated with this system is unitarily equivalent to $\Gamma$. If $\operatorname{Ker} \Gamma=\{0\}$, then $R=\Gamma$ and $T$ is unitarily equivalent to the backward shift $S^{*}$. In this case the vectors $\left\{\left(T^{*}\right)^{j} q\right\}_{j \geqslant 0}$ form an orthonormal basis in $\mathcal{K}$ and $R$ has Hankel matrix $\left\{\alpha_{j+k}\right\}_{j, k \geqslant 0}$ in this basis. It is easy to see that the operator $\Lambda$ also has a Hankel matrix (namely $\left\{\alpha_{j+k+1}\right\}_{j, k \geqslant 0}$ ) in the same basis. So $\Lambda$ must satisfy the same conditions (C1)-(C3).

If $\operatorname{Ker} \Gamma \neq\{0\}$, then it follows from the remark to Theorem 2.1 that $T$ is unitarily equivalent to the restriction of $S^{*}$ to $(\operatorname{Ker} \Gamma)^{\perp}$. It is easy to see that in this case $\Lambda=$ $S^{*} \Gamma \mid(\operatorname{Ker} \Gamma)^{\perp}$ and the operator $S^{*} \Gamma$ is a Hankel operator whose kernel is contained in $\operatorname{Ker} \Gamma$.

Therefore in both cases the spectral multiplicity function $\nu_{\Lambda}$ of the operator $\Lambda$ must satisfy the condition (C3).

A natural question can arise if we look at our definition of $\Lambda$. The operator $\Lambda$ was defined with the help of multiplication by the function $\tilde{\mathcal{J}}$. It is easy to see from (5.4) that for $t \in \sigma_{0} \cap \Delta$

$$
\nu_{\Lambda}(t)-\nu_{\Lambda}(-t)=2
$$

Hence if the restriction of the measure $\lambda$ to the set $\sigma_{0} \cap \Delta$ contains a non-zero singular component, then (10.1) contradicts the condition (C3). However this can never happen: the singular components of the measures $\varrho$ and $\lambda$ are mutually singular and so the restriction of $\lambda$ to $\sigma_{0} \cap \Delta$ is absolutely continuous. This follows from the following Aronszajn-Donoghue theorem (see [RS, §XIII.6]). 
THEOREM 10.1. Let $K$ be a self-adjoint operator on Hilbert space and let

$$
L=K+(\cdot, r) r,
$$

where $r$ is a cyclic vector of $K$. Then the singular components of the spectral measures of $L$ and $R$ are mutually singular.

Theorem 10.1 has an elementary proof. However it is possible to deduce it from our results on Hankel operators.

Proof of Theorem 10.1. Without loss of generality we can assume that both $L$ and $K$ are positive and invertible. Since $K$ is cyclic, it is unitarily equivalent to multiplication by the independent variable on $L^{2}(\varrho)$, where $\varrho$ is a positive Borel measure with compact support in $(0, \infty)$. Let $\Gamma$ be the orthogonal sum of $K^{1 / 2},-K^{1 / 2}$ and the zero operator on an infinite-dimensional space. Clearly $\Gamma$ satisfies the conditions (C1)-(C3). Let $R=$ $\Gamma \mid(\operatorname{Ker} \Gamma)^{\perp}$ and $W=|R|$. Then $W$ is unitarily equivalent to $K^{1 / 2} \oplus K^{1 / 2}$. We can now put $p=r, q_{0}=W^{-1} x$ (in this case $W$ is invertible). Without loss of generality we can assume that $\left\|q_{0}\right\|_{L^{2}(\ell)}=1$. We can now define the operators $J, \mathcal{J}, \Lambda$ as above. In our case $\sigma(W)=\sigma_{0}$ and so the operator $A^{*}$ defined by (3.1) is asymptotically stable for any choice of a cyclic vector $q_{0}$ of $W$. (Note that in our case the proof of the asymptotic stability of $A^{*}$ is considerably simpler than in the general case.)

Assume that the singular components of the measures $\varrho$ and $\lambda$ are not mutually singular. In this case the restriction of $\lambda$ to $\Delta$ (see §III.5) has a non-zero singular component. It follows from (5.4) that

$$
\nu_{\lambda}(t)=2, \quad \nu_{\lambda}(-t)=0, \quad \lambda_{s} \text {-a.e. on } \Lambda .
$$

However, as we have already observed, $\nu_{\Lambda}$ must satisfy (C3) and so

$$
\left|\nu_{\Lambda}(t)-\nu_{\Lambda}(-t)\right| \leqslant 1, \quad \lambda_{s} \text {-a.e. }
$$

which contradicts $(10.2)$.

Remark. Note that it is also possible to deduce Theorem 1.1 in Chapter I from the above Theorem 10.1 . 


\section{References}

[BS1] Birman, M.Sh. \& Solomyak, M.Z., Spectral Theory of Self-Adjoint Operators in Hilbert Space. Reidel, Dordrecht, 1987.

[BS2] - Operator integration, perturbations, and commutators. Zap. Nauchn. Sem. Leningrad. Otdel. Mat. Inst. Steklov. (LOMI), 170 (1989), 34-56 (Russian).

[C] Clark, D. N., On the spectra of bounded Hermitian Hankel matrices. Amer. J. Math., $90(1968), 627-656$.

[D1] Douglas, R.G., On majorization, factorization and range inclusion of operators on Hilbert space. Proc. Amer. Math. Soc., 17 (1966), 413-415.

[D2] - Banach Algebra Techniques in Operator Theory. Academic Press, New York, 1972.

[F] Francis, B. A., A Course in $H_{\infty}$ Control Theory. Lecture Notes in Control and Inform. Sci., 88. Springer-Verlag, Berlin-New York, 1987.

[G] Glover, K., All optimal Hankel-norm approximations of linear multivariable systems and their $L^{\infty}$-error bounds. Internat. J. Control, 39 (1984), 1115-1193.

[Ho1] Howland, J.S., Spectral theory of self-adjoint Hankel matrices. Michigan Math. J., 33 (1986), 145-153.

[Ho2] - Spectral theory of operators of Hankel type. I. Preprint, 1991.

[Ho3] - Spectral theory of operators of Hankel type. II. Preprint, 1991.

[HW] Helton, J. W. \& Woerdeman, H. J., Symmetric Hankel operators: minimal norm extensions and eigenfunctions. Preprint, 1990.

[Ka] Kato, T., Perturbation Theory for Linear Operators. Springer-Verlag, Berlin-New York, 1966.

[KP] Khrushchev, S. V. \& Peller, V. V., Moduli of Hankel operators, past and future, in Linear and Complex Analysis Problem Book. Lecture Notes in Math., 1043, pp. 92-97. Springer-Verlag, Berlin-New York, 1984.

[N] Nikol'skiI, N. K., Treatise on the Shift Operator. Spectral Function Theory. SpringerVerlag, Berlin-Heidelberg, 1986.

[O1] Ober, R. J., A note on a system theoretic approach to a conjecture by Peller-Khrushchev. Systems Control Lett., 8 (1987), 303-306.

[02] - A note on a system theoretic approach to a conjecture by Peller-Khrushchev: the general case. IMA J. Math. Control Inform., 7 (1990), 35-45.

[OM] Ober, R. J. \& MONTGOMERY-Smith, S., Bilinear transformation of infinite-dimensional state-space systems and balanced realizations of non-rational transfer functions. SIAM J. Control Optim., 28 (1990), 438-465.

[Pa] Partington, J. R., An Introduction to Hankel Operators. London Math. Soc. Stud. Texts, 13. Cambridge Univ. Press, Cambridge, 1988.

[Pe] Peller, V. V., Hankel operators and continuity properties of best approximation operators. Algebra i Analiz, 2:1 (1990), 163-189; English translation in Leningrad Math. J., 2 (1991), 139-160.

[PK] Peller, V. V. \& Khrushchev, S. V., Hankel operators, best approximations, and stationary Gaussian processes. Uspekhi Mat. Nauk, 37:1 (1982), 53-126; English translation in Russian Math. Surveys, 37:1 (1982), 61-144.

[Po] Power, S. C., Hankel Operators on Hilbert Space. Research Notes in Math., 64. Pitman, Boston, i. 1982.

[RS] READ, M. \& Simon, B., Methods of Modern Mathematical Physics IV: Analysis of Operators. Academic Press, New York-London, 1978.

[S] Salamon, D., Realization theory in Hilbert space. Math. Systems Theory, 21 (1989), $147-164$. 
[SF] Sz.-NAGY, B. \& FoIAS, C., Harmonic Analysis of Operators on Hilbert Space. Akadémiai Kiadó, Budapest, 1970.

[T1] Treil, S.R., Moduli of Hankel operators and a problem of Peller-Khrushchev. Dokl. Akad. Nauk SSSR, 283 (1985), 1095-1099; English translation in Soviet Math. Dokl., $32(1985), 293-297$.

[T2] - The inverse spectral problem for the modulus of Hankel operator and balanced realizations. Algebra $i$ Analiz, 2:2 (1990), 158-182; English translation in Leningrad Math. J., 2 (1991), 353-375.

[TV] Treil, S.R. \& VASYunin, V.I., The inverse spectral problem for the modulus of a Hankel operator. Algebra i Analiz, 1:4 (1989), 54-67; English translation in Leningrad Math. J., 1 (1990), 859-870.

[Y] Young, N. J., Balanced realizations in infinite dimensions, in Operator Theory and Systems (Amsterdam, 1985). Oper. Theory: Adv. Appl., 19, pp. 449-471. Birkhäuser, Basel, 1976.

\author{
A. V. MEGRETSKII \\ Iowa State University \\ Ames, IA 50011 \\ U.S.A. \\ alex@iastate.edu \\ V.V. PELLER \\ Department of Mathematics \\ Kansas State University \\ Manhattan, KS 66506 \\ U.S.A. \\ peller@math.ksu.edu \\ S. R. TREIL \\ Department of Mathematics \\ Michigan State University \\ East Lansing, MI 48824 \\ U.S.A. \\ treil@math.msu.edu
}

Department of Electrical Engineering and Computer Science

Received April 13, 1993 